\title{
High moments of the Estermann function
}

\author{
Sandro Bettin
}

\begin{abstract}
For $a / q \in \mathbb{Q}$ the Estermann function is defined as $D(s, a / q):=\sum_{n \geq 1} d(n) n^{-s} \mathrm{e}\left(n \frac{a}{q}\right)$ if $\Re(s)>1$ and by meromorphic continuation otherwise. For $q$ prime, we compute the moments of $D(s, a / q)$ at the central point $s=1 / 2$, when averaging over $1 \leq a<q$.

As a consequence we deduce the asymptotic for the iterated moment of Dirichlet $L$ functions $\sum_{\chi_{1}, \ldots, \chi_{k}(\bmod q)}\left|L\left(\frac{1}{2}, \chi_{1}\right)\right|^{2} \cdots\left|L\left(\frac{1}{2}, \chi_{k}\right)\right|^{2}\left|L\left(\frac{1}{2}, \chi_{1} \cdots \chi_{k}\right)\right|^{2}$, obtaining a power saving error term.

Also, we compute the moments of certain functions defined in terms of continued fractions. For example, writing $f_{ \pm}(a / q):=\sum_{j=0}^{r}( \pm 1)^{j} b_{j}$ where $\left[0 ; b_{0}, \ldots, b_{r}\right]$ is the continued fraction expansion of $a / q$ we prove that for $k \geq 2$ and $q$ primes one has $\sum_{a=1}^{q-1} f_{ \pm}(a / q)^{k} \sim 2 \frac{\zeta(k)^{2}}{\zeta(2 k)} q^{k}$ as $q \rightarrow \infty$.
\end{abstract}

\section{Introduction}

Since the pioneering work of Hardy and Littlewood [HL], the study of moments of families of L-functions has gained a central role in number theory. This is mostly due their numerous applications on, e.g., non-vanishing (see [IS], Sou00]) and sub-convexity estimates (cf. [CI]). Moreover, moments are also important as they highlight clearly the symmetry of each family.

In this paper we consider the moments of the Estermann function at the central point and, as a consequence, we obtain new results for moments of Dirichlet $L$-functions. We will describe the Estermann function in Section 1.1.2, we now focus on the the family of Dirichlet $L$-functions. For this family only the second and fourth moments have been computed. The asymptotic for the second moment was obtained by Paley [Pal], whereas Heath-Brown [H-B81] considered the fourth moment and showed

$$
\frac{1}{\varphi^{*}(q)} \sum_{\chi(\bmod q)}^{*}|L(1 / 2, \chi)|^{4} \sim \frac{1}{2 \pi^{2}} \prod_{p \mid q} \frac{(1-1 / p)^{3}}{1+1 / p}(\log q)^{4}
$$

2010 Mathematics subject classification. 11M06, 11M41, 11A55 (primary), 11N75, 11N75 (secondary)

Key words and phrases. Estermann function, Dirichlet L-functions, divisor function, continued fractions, mean values, moments. 
provided that $q$ doesn't have "too many prime divisors", a restriction that was later removed by Soundararajan [Sou07]. As usual, $\sum^{*}$ indicates that the sum is restricted to primitive characters and $\varphi^{*}(q)$ denotes the number of such characters. The problem of computing the full asymptotic expansion for the fourth moment was later solved by Young [You11b] in the case when $q$ is prime. He proved

$$
\frac{1}{\varphi^{*}(q)} \sum_{\chi(\bmod q)}^{*}|L(1 / 2, \chi)|^{4}=\sum_{i=0}^{4} c_{i}(\log q)^{i}+O\left(q^{-\frac{5}{512}+\varepsilon}\right)
$$

for some absolute constants $c_{i}$ with $c_{4}=\left(2 \pi^{2}\right)^{-1}$. Recently, Blomer, Fouvry, Kowalski, Michel and Milićević [BFKMM] introduced several improvements in Young's work improving the error term in (1.2) to $O\left(q^{-\frac{1}{32}+\varepsilon}\right)$.

In this paper, we consider a variation of this problem and compute the asymptotic of

$$
M_{k}(q)=\frac{1}{\varphi^{*}(q)^{k-1}} \sum_{\chi_{1}, \ldots, \chi_{k-1}(\bmod q)}^{*}\left|L\left(1 / 2, \chi_{1}\right)\right|^{2} \cdots\left|L\left(1 / 2, \chi_{k-1}\right)\right|^{2}\left|L\left(1 / 2, \chi_{1} \cdots \chi_{k-1}\right)\right|^{2},
$$

where the sum has the extra restriction that $\chi_{1} \cdots \chi_{k-1}$ is primitive. If $k=2$, this coincides with the usual 4-th moment of Dirichlet $L$-functions as computed by Young, whereas if $k>2$ then $M_{k}(q)$ should be thought of as an iterated 4-th moment, since each character appears 4 times in the above expression. We shall prove the following theorem.

Theorem 1. Let $k \geq 3$ and let $q$ be prime. Then, there exists an absolute constant $A>0$ such that

$$
M_{k}(q)=\sum_{n=1}^{\infty} \frac{2^{\nu(n)}}{n^{\frac{k}{2}}}\left(\left(\log \frac{q}{8 n \pi}\right)^{k}+(-\pi)^{k}\right)+O_{\varepsilon}\left(k^{A k} q^{-\delta_{k}+\varepsilon}\right),
$$

where $\nu(n)$ is the number of different prime factors of $n, \delta_{k}:=\frac{k-2-3 \vartheta}{2 k+5}$ with $\vartheta=\frac{7}{64}$ being the best bound towards Selberg's eigenvalue conjecture. Also, the implicit constant depends on $\varepsilon$ only.

Remark 1. Notice that $\delta_{k}$ is a decreasing sequence such that $\delta_{k} \rightarrow \frac{1}{2}$ as $k \rightarrow \infty$. For $\vartheta=\frac{7}{64}$ the first few values of $\delta_{k}$ are $\delta_{3}=\frac{43}{704}, \delta_{4}=\frac{107}{832}, \delta_{5}=\frac{57}{320}$.

Theorem 1 yields an asymptotic formula for $M_{k}(q)$ for $k<\eta \frac{\log q}{\log \log q}$ with $\eta>0$ sufficiently small. Larger values of $k$ are easier to deal with and one obtains the following corollary.

Corollary 1. Let $q$ be prime. Then as $q \rightarrow \infty$ we have

$$
M_{k}(q) \sim \frac{\zeta\left(\frac{k}{2}\right)^{2}}{\zeta(k)}(\log (q / 8 \pi)+\gamma)^{k}
$$

uniformly in $3 \leq k=o\left(q^{\frac{1}{2}} \log q\right)$. Moreover this range is optimal, meaning that (1.4) is false if $k \gg q^{\frac{1}{2}} \log q$. 
A moment somewhat similar to (1.3) was previously considered by Chinta Chi] who used a multiple Dirichlet series approach to compute the asymptotic of the first moment of (roughly)

$$
L\left(1 / 2, \chi_{d_{1}}\right) L\left(1 / 2, \chi_{d_{2}}\right) L\left(1 / 2, \chi_{d_{1}} \chi_{d_{2}}\right),
$$

where $\chi_{d}$ denotes the quadratic character associated to the extension $\mathbb{Q}(\sqrt{d})$ of $\mathbb{Q}$. We remark there is a big difference between (1.3) and this case. Indeed, if $\chi_{1}, \chi_{2}$ are characters modulo $q$ then so is $\chi_{1} \chi_{2}$, whereas if $d_{1}, d_{2} \approx X$ then $\chi_{d_{1}} \chi_{d_{2}}$ is typically a character with conductor $\approx X^{2}$. This means that (1.3) roughly correspond to an iterated 4-th moment, whereas the second moment of (1.5) roughly correspond to an iterated 6-th moment of quadratic Dirichlet $L$-functions, and thus it doesn't seem to be attackable with the current technology. (As a comparison, the first moment computed by Chinta roughly correspond to an iterated 3-rd moment).

\subsection{Twisted moments, the Estermann function, and continued frac- tions}

A nice feature of Theorem 1 is that it can be essentially rephrased in terms of high moments of other functions appearing naturally in Number Theory. Indeed, the same computations give also the asymptotic for moments of twisted moments of Dirichlet $L$-functions, of the Estermann function, and of certain functions defined in terms of continued fractions. We now briefly describe each of this objects and give the corresponding version of Theorem 1 .

\subsubsection{Moments of twisted moments}

Several classical methods to investigate the central values of Dirichlet $L$-functions pass through the study of the second moment of $L(s, \chi)$ times a Dirichlet polynomial $P_{\theta}(s, \chi):=\sum_{n \leq q^{\theta}} a_{n}$. $\chi(n) n^{-s}$ :

$$
\frac{1}{\varphi^{*}(q)} \sum_{\chi(\bmod q)}^{*}\left|L(1 / 2, \chi) P_{\theta}(1 / 2, \chi)\right|^{2} .
$$

For example, Iwaniec and Sarnak proved that $1 / 3$ of the Dirichlet $L$-functions do not vanish at the central point via proving the asymptotic for such average for $\theta<1 / 2$ (and choosing $P_{\theta}$ to be a mollifier). Moreover, it is easy to see that if one could extend such asymptotic to all polynomials of length $\theta<1$, then the Lindelöf hypothesis would follow.

Expanding the square, using the multiplicativity of Dirichlet characters, and renormalizing, one immediately sees that (1.6) can be reduced to an average of twisted moments of the form

$$
M(a, q):=\frac{q^{\frac{1}{2}}}{\varphi^{*}(q)} \sum_{\chi(\bmod q)}^{*}\left|L\left(\frac{1}{2}, \chi\right)\right|^{2} \chi(a),
$$


for $(a, q)=1$. By the orthogonality of Dirichlet characters one can immediately rewrite Theorem 1 (and (1.1) ) in terms of $M(a, q)$. In particular, one has

$$
\sum_{a=1}^{q} M(a, q)^{k}=q^{\frac{k}{2}} M_{k}(q) \sim \begin{cases}\frac{\zeta\left(\frac{k}{2}\right)^{2}}{\zeta(k)}(\log (q / 8 \pi)+\gamma)^{k} & \text { if } 3 \leq k=o\left(q^{\frac{1}{2}} \log q\right) \\ \frac{1}{2 \pi^{2}}(\log q)^{4} & \text { if } k=2\end{cases}
$$

as $q \rightarrow \infty$ with $q$ prime.

\subsubsection{Moments of the Estermann function}

For $(a, q)=1, q>0, \alpha, \beta \in \mathbb{C}$ and $\Re(s)>1-\min (\Re(\alpha), \Re(\beta))$, the Estermann function is defined as

$$
D_{\alpha, \beta}(s, \alpha, a / q):=\sum_{n=1}^{\infty} \mathrm{e}(n a / q) \frac{\tau_{\alpha, \beta}(n)}{n^{s}}=D_{\cos ; \alpha, \beta}(s, a / q)+i D_{\sin ; \alpha, \beta}(s, a / q),
$$

where $D_{\cos }$ and $D_{\text {sin }}$ have the same definition of $D$, but with e $(n a / q)$ replaced by $\cos (2 \pi n a / q)$ and $\sin (2 \pi n a / q)$ respectively. As usual, $\mathrm{e}(x):=e^{2 \pi i x}$ and $\tau_{\alpha, \beta}(n):=\sum_{d_{1} d_{2}=n} d_{1}^{-\alpha} d_{2}^{-\beta}$.

$D_{\alpha, \beta}(s, a / q$ ) was first introduced (with $\alpha=\beta=0$ ) by Estermann who proved that it extends to a meromorphic function on $\mathbb{C}$ satisfying a functional equation relating $D_{\alpha, \beta}(s, a / q)$ with $D_{-\alpha,-\beta}(1-s, \pm \bar{a} / q)$, where $\bar{a}$ denotes the inverse of $a$ modulo $q$ (and similarly for $D_{\sin }$ and $D_{\cos }$ which satisfy a more symmetric functional equation given by (3.2) below).

Since the work of Estermann [Est30, Est32] on the number of representations of an integer as a sum of two or more products, the Estermann function has proved itself as a valuable tool when studying additive problems of similar flavor (see e.g. [Mot80, Mot94]) and in problems related to moments of $L$-functions (see e.g. [H-B79, You11b] and [CGG]). These applications mainly use the functional equation for $D$ as it encodes Voronoi's summation in an analytic fashion, allowing for a simpler computation of the main terms. However, the Estermann function is an interesting object by its own right, due to its surprising symmetries (see [Bet16]) and to the connections with some interesting objects in analytic number theory. For example, by the work of Ishibashi [Ish] (see also [BC13b]) one has

$$
D_{\mathrm{sin} ; 1,0}(0, a / q)=\pi \mathrm{s}(a, q), \quad D_{\sin ; 0,0}(0, a / q)=\frac{1}{2} \mathrm{c}_{0}(a / q),
$$

where $\mathrm{s}(a, q)$ is the classical Dedekind sum and $\mathrm{c}_{0}(a / q)$ is a cotangent sum, related to the Nymann-Beurling criterion for the Riemann hypothesis, which has been object of intensive studies in recent years (see, for example, [BC13a, [MR, Bet15]). Ishibashi obtained similar identities also for other values of $\alpha, \beta$, and in particular if $\alpha$ is a positive odd integer one obtains that $D_{\sin ; \alpha, 0}(0, a / q)$ is related to certain Dedekind cotangent sums studied by Beck [Bec]. All these functions satisfy certain reciprocity relations and provide examples of "quantum modular forms" (cf. Zag ). 
Moreover, one can also obtain formulae relating the Estermann function to twisted moments of Dirichlet $L$-function (see [Bet16] and [CG]) and in particular for $q$ prime and $(a, q)=1$, one has

$$
D_{\cos ; 0,0}\left(\frac{1}{2}, a / q\right)+D_{\sin ; 0,0}\left(\frac{1}{2}, a / q\right)=M(a, q)+\frac{2\left(q^{\frac{1}{2}}-1\right)}{\varphi(q)} \zeta\left(\frac{1}{2}\right)^{2} .
$$

By this formula and (1.7), it is clear that Theorem 1 gives an asymptotic formula for the high moments of $D_{\cos ; 0,0}(1 / 2, a / q)+D_{\sin ; 0,0}(1 / 2, a / q)$. The method however allows one to obtain the asymptotic for the joint moments of $D_{\text {cos; } 0,0}(1 / 2, a / q)$ and $D_{\text {sin;0,0 }}(1 / 2, a / q)$. We shall state this in Theorem 4 below where shifts are also included (all our results will be derived from this theorem). Here we content ourselves with giving the asymptotic for the high moments of the Estermann function:

Theorem 2. Let q be prime. Then,

$$
\frac{1}{\varphi(q)} \sum_{a=1}^{q-1} D_{0,0}\left(\frac{1}{2}, \frac{a}{q}\right)^{k} \sim q^{\frac{k}{2}-1} 2^{1-\frac{k}{2}} \frac{\zeta\left(\frac{k}{2}\right)^{2}}{\zeta(k)} \Re\left(\left(e^{\frac{\pi i}{4}}\left(\log \frac{q}{8 \pi}+\gamma\right)-e^{-\frac{\pi i}{4} \frac{\pi}{2}}\right)^{k}\right)
$$

as $q \rightarrow \infty$, uniformly in $3 \leq k=o\left(q^{\frac{1}{2}} \log q\right)$. In particular, if $3 \leq k \ll 1$ then

$$
\frac{1}{\varphi(q)} \sum_{a=1}^{q-1} D_{0,0}\left(\frac{1}{2}, \frac{a}{q}\right)^{k} \sim q^{\frac{k}{2}-1} 2^{1-\frac{k}{2}} \frac{\zeta\left(\frac{k}{2}\right)^{2}}{\zeta(k)}\left(\cos \left(\frac{k \pi}{4}\right)(\log q)^{k}-\frac{\pi}{2} \sin \left(\frac{k \pi}{4}\right)(\log q)^{k-1}\right)
$$

as $q \rightarrow \infty$.

Theorem 2 should be compared with Theorem 1.2 of [FGKM] which gives the asymptotic for the moments of

$$
\sum_{\substack{n \geq 1, n \equiv a(\bmod q)}} d(n) f(n / X)-\mathcal{M}_{1}(X, q)
$$

where $\mathcal{M}_{1}(X, q)$ is a certain main term, $f \in \mathcal{C}_{0}^{\infty}\left(\mathbb{R}^{+}\right)$, and $X=q^{2} / \Phi(q)$ with $\Phi(x) \rightarrow \infty$ and $\Phi(x) \ll x^{\varepsilon}$ as $x \rightarrow \infty$ (see also [LY] the case of sharp cut-offs). For comparison, Theorem 2 should be thought of roughly computing the moments of

$$
\sum_{n \geq 1} d(n) \mathrm{e}(n a / q) f(n / X)-\mathcal{M}_{2}(X, q)
$$

with $X \approx q$ and another main term $\mathcal{M}_{2}(X, q)$. Despite some superficial similarities, the two problems are very different in nature (and have very different proofs). For example, a first important difference is that the range of the summation in (1.10) is twice as long as that of (1.11) in the logarithmic scale. 


\subsubsection{Moments of certain functions defined in terms of continued fractions}

Finally, we discuss the relation with continued fractions. In [Bet16] (see also [You11a]), it was observed that $M(a, q)$, and more generally, $D_{\cos }$ and $D_{\sin }$, can be written in terms of the continued fraction expansion of $a / q$. Indeed, if $a, q \in \mathbb{Z}_{>0}$ and $\left[b_{0} ; b_{1}, \cdots, b_{\kappa}, 1\right]$ is the continued fraction expansion of $\frac{a}{q}$, then for $q$ prime one has

$$
M(a, q)=\sum_{\substack{j=1, j \text { odd }}}^{\kappa} b_{j}^{\frac{1}{2}}\left(\log \frac{b_{j}}{8 \pi}+\gamma\right)-\frac{\pi}{2} \sum_{\substack{j=1, j \text { even }}}^{\kappa} b_{j}^{\frac{1}{2}}+O(\log q) .
$$

It is therefore not surprising that Theorem 1 have an incarnation also in terms of moments for functions of the rationals defined as

$$
f_{r, \pm}(a / q):=\sum_{j=1}^{\kappa}( \pm 1)^{j} b_{j}^{\frac{r}{2}}
$$

where $r \in \mathbb{Z}_{\geq 1}$.

Theorem 3. Le $q$ be prime and let $k, r \in \mathbb{Z}_{\geq 1}$ with $3 \leq k r=o\left(\frac{\log q}{\log \log q}\right)$. Then

$$
\sum_{a=1}^{q} f_{r, \pm}(a / q)^{k} \sim 2 \frac{\zeta\left(\frac{k r}{2}\right)^{2}}{\zeta(k r)} q^{\frac{k r}{2}}
$$

as $q \rightarrow \infty$.

Starting with the work of Heilbronn [Hei], who considered the average value of $f_{0,+}$, there have been a very large number of papers computing the mean values of functions defined in terms of the continued fraction expansion. In particular, we cite the works [Por, Ton] on $f_{0,+}$ and [YK] where the asymptotic for the first moment of $f_{2,+}$ was given. However, to the knowledge of the author, Theorem 3 is the first result giving asymptotic formulae for $k$-th moments with $k \geq 3$ without exploiting an extra average over $q$ (as in [Hen, BV]). For $k=2$ the only cases previously known where obtained by Bykovskii $\mathrm{Byk}$ (considering the second moment of $f_{0,+}$ ) and by the author [Bet16] (considering the second moment of a variation of $\left.f_{2,+}\right)$. By combining the techniques employed in [Bet16] and in this paper it seems possible to extend Theorem 3 to more general functions of similar shape.

\subsection{Brief outline of the proof}

The approximate functional equation allows one to express $M_{k}(q)$ roughly in the form

$$
\sum_{\substack{ \pm n_{1} \pm 2 \cdots \pm n_{k} \equiv 0(\bmod q), n_{1} \cdots n_{k} \ll q^{k}}} \frac{d\left(n_{1}\right) \cdots d\left(n_{k}\right)}{n_{1}^{\frac{1}{2}} \cdots n_{k}^{\frac{1}{2}}},
$$


so that the problem of estimating $M_{k}(q)$ reduces to that of computing the asymptotic for this quadratic divisor problem. The diagonal terms (i.e. the terms with $\pm n_{1} \pm_{2} \cdots \pm n_{k}=0$ ) are a bit easier to study and give a main term; the main difficulties then lie in obtaining an asymptotic for the off-diagonal terms and in assembling the various main terms. In his proof of (1.2), which corresponds to (1.13) with $k=2$, Young used a combination of several techniques each effective for some range of the variables $n_{1}, n_{2}$. In particular, when $n_{1} \approx n_{2}$ (in the logarithmic scale) he followed an approach à la Motohashi [Mot97] using Kuznetsov formula, whereas when one variable is much larger than the other one, he used (new) estimates for the average value of the divisor function in arithmetic progressions.

Our approach is similar to that of Young, however there are several substantial differences which we will now discuss in some detail. First, the larger number of variables gives us the advantage of having to deal with more "flexible" sums enlarging the ranges where the various estimates are effective. For this reason, we can afford to use slightly weaker bounds employing the spectral theory only indirectly, through the bounds of Deshouilliers and Iwaniec [DI] (together with Kim and Sarnak's bound for the exceptional eigenvalues [Kim]). It seems likely that one could use spectral methods in a more direct and efficient way, however the generalization of the methods in [You11b] (or [BFKMM]) to the $k \geq 3$ case is not straightforward and so we choose a simpler route as this is still sufficient for our purposes.

The larger number of variables has also a cost. Indeed, it introduces several new complications in the extraction and in the combination of the main terms, a process that requires a rather careful analysis and constitutes the central part of this paper. One of the causes of the complicated shape of the main terms (cf. (6.1)-(6.2)) is that with more than two variables the dichotomy "either one variable is much bigger than the other or the variables have the same size" doesn't hold for $k>2$ and one has to (implicitly) deal also with cases such as $n_{1} \approx \cdots \approx n_{k-1} \approx q^{1+1 / k}$ and $n_{k} \approx 1$.

Another difference with Young's work arises when studying the diagonal terms. If $k=2$, then one can handle these terms easily thanks to Ramanujan's formula $\sum_{n \geq 1} d(n)^{2} / n^{s}=$ $\zeta(s)^{4} / \zeta(2 s)$. If $k \geq 3$, we don't have such a nice exact formula, and we are left with the problem of showing that the series

$$
\sum_{ \pm n_{1} \pm \cdots \pm n_{k}=0} \frac{d\left(n_{1}\right) \cdots d\left(n_{k}\right)}{\left(n_{1} \cdots n_{k}\right)^{s}}
$$

can be meromorphically continued past the line $\Re(s)=1-1 / k$ which is the boundary of convergence. We shall leave this problem to a different paper, Bet], where with similar (but a bit simpler) techniques we prove that this series admits meromorphic continuation to the region $\Re(s)>1-2 /(k+1)$.

Last, we mention a more technical problem. One of the steps in Young's proof requires separating $n_{1}, n_{2}$ in expressions of the form $\left(n_{1} \pm n_{2}\right)^{-z}$ when $\Re(z) \approx 0$. This can be easily obtained by using some classical Mellin formulae; however, whereas the Mellin integral corresponding to $(1+x)^{-z}$ converges absolutely, the Mellin integral corresponding to $(1-x)^{-z}$ 
converges only conditionally so that the terms containing $\left(n_{1}-n_{2}\right)^{-z}$ demand some caution. In our case this problem becomes rather more subtle as we need to apply these formulae iteratively in order to handle expressions such as $\left(n_{1} \pm \cdots \pm n_{k}\right)^{-s}$. We overcome this difficulties by using a modification of the resulting "iterated" Mellin formula allowing us to write such expressions in terms of absolutely convergent integrals (see Section 10 for the details).

\subsection{The structure of the paper}

The paper is organized as follow. In Section 2 we state Theorem 4, a more general version of Theorem 2 providing the asymptotic for the mixed moments of $D_{\cos }$ and $D_{\sin }$ (as well as allowing for some small shifts). We then use this result to deduce Theorem 1, 21 and 3, In Section 3 we give some lemmas on the Estermann function which we shall need later on. It is in this lemmas that the spectral theory comes (indirectly) into play. The proof of Theorem 4 is carried out in Sections 5,9, after introducing some notation in Section 4, and constitutes the main body of the paper. Finally, in Section 10 we will prove the Mellin formula mentioned at the end of the previous section as well as some technical Lemmas needed in order to use this formula effectively.

\section{Acknowledgments}

The author would like to thank Sary Drappeau, Dimitris Koukoulopoulos and Maksym Radziwiłł for helpful discussions.

This paper was started while the author was a postdoc at the Centre de Recherche Mathématiques (CRM) in Montréal, and was completed during another visit at the same institution. The author is grateful to the CRM for the hospitality and for providing a stimulating working environment.

The work of the author is partially supported by FRA 2015 "Teoria dei Numeri" and by PRIN "Number Theory and Arithmetic Geometry".

\section{Mixed moments of $D_{\cos }$ and $D_{\sin }$ and the deduction of the main theorems}

Let $k \geq 1, q$ be a prime and let $\alpha_{1}, \ldots, \alpha_{k}, \beta_{1}, \ldots, \beta_{k} \in \mathbb{C}$. Then, for any subset $\Upsilon \subseteq\{1, \ldots, k\}$ let $M_{\Upsilon, k}$ be the mixed shifted moment

$$
M_{\Upsilon, k}:=\frac{1}{\varphi(q)} \sum_{a=1}^{q-1} \prod_{i=1}^{k} D_{i ; \alpha_{i}, \beta_{i}}\left(\frac{1}{2}, \frac{a}{q}\right),
$$


where $D_{i ; \alpha_{i}, \beta_{i}}:=D_{\sin ; \alpha_{i}, \beta_{i}}$ if $i \in \Upsilon$ and $D_{i}:=D_{\cos ; \alpha_{i}, \beta_{i}}$ otherwise. Also, let

$$
\Gamma_{i}(s):= \begin{cases}\Gamma\left(\frac{1}{2}+s\right) & \text { if } i \in \Upsilon \\ \Gamma(s) & \text { otherwise }\end{cases}
$$

Since $D_{\sin ; \alpha_{i}, \beta_{i}}\left(s,-\frac{a}{q}\right)=-D_{\sin ; \alpha_{i}, \beta_{i}}\left(s, \frac{a}{q}\right)$, then $M_{\Upsilon, k}$ is identically zero if $|\Upsilon|$ is odd. If $|\Upsilon|$ is even the asymptotic for $M_{\Upsilon, k}$ is given by the following theorem, provided that $k \geq 3$ (the corresponding theorem for $k=2$ is essentially implicit in You11b], whereas the case $k=1$ is trivial).

Theorem 4. Let $\Upsilon \subseteq\{1, \ldots, k\}$ with $|\Upsilon|$ even. Let $k \geq 3$ and let $q$ be a prime. Let $\boldsymbol{\alpha}=\left(\alpha_{1}, \ldots, \alpha_{k}\right), \boldsymbol{\beta}:=\left(\beta_{1}, \ldots, \beta_{k}\right) \in \mathbb{C}^{k}$ with $\left|\alpha_{i}\right|,\left|\beta_{i}\right| \ll \frac{1}{\log q},\left|\alpha_{i}\right|,\left|\beta_{i}\right| \leq 1 / 10$ and $\alpha_{i} \neq \beta_{i}$ for all $i=1, \ldots, k$. Then, there exists an absolute constant $A>0$ such that for any $\varepsilon>0$ we have

$$
M_{\Upsilon, k}=\sum_{\left\{\alpha_{i}^{\prime}, \beta_{i}^{\prime}\right\}=\left\{\alpha_{i}, \beta_{i}\right\}} \mathscr{M}_{\boldsymbol{\alpha}^{\prime}, \boldsymbol{\beta}^{\prime}}+O_{\varepsilon}\left(k^{A k} q^{\frac{k}{2}-1-\delta_{k}+\varepsilon}\right)
$$

where $\delta_{k}:=\frac{k-2-3 \vartheta}{2 k+5}$

$$
\mathscr{M}_{\boldsymbol{\alpha}, \boldsymbol{\beta}}:=\frac{q^{\frac{k}{2}-1}}{2^{k-1}} \frac{\zeta\left(\frac{k}{2}-\sum_{i=1}^{k} \alpha_{i}\right) \zeta\left(\frac{k}{2}+\sum_{i=1}^{k} \beta_{i}\right)}{\zeta\left(k-\sum_{i=1}^{k}\left(\alpha_{i}-\beta_{i}\right)\right)} \prod_{i=1}^{k} \frac{\Gamma_{i}\left(\frac{1}{4}-\frac{\alpha_{i}}{2}\right)}{\Gamma_{i}\left(\frac{1}{4}+\frac{\alpha_{i}}{2}\right)}\left(\frac{q}{\pi}\right)^{-\alpha_{i}} \zeta\left(1-\alpha_{i}+\beta_{i}\right)
$$

and where the implicit constant in the error term depends on $\varepsilon$ only.

Remark 2. If $\alpha_{i}=\beta_{i}$ for some $i=1, \ldots k$, then $\mathcal{M}_{\boldsymbol{\alpha}, \boldsymbol{\beta}}$ has to be interpreted as the limit for $\alpha_{i} \rightarrow \beta_{i}$ (cf. (2.4) below).

As mentioned in Section 1.3, we will prove Theorem 44 in Sections 5.9. We will now deduce Theorem 1, 2, 3 from Theorem 4.

\subsection{Proof of Theorem [1, 2 and 3 and of Corollary 1}

We start by observing that if $|\Upsilon|$ is even then from Theorem 4 one has

$$
\sum_{a=1}^{q-1} \prod_{i=1}^{k} D_{i ; 0,0}\left(\frac{1}{2}, \frac{a}{q}\right)=\frac{q^{\frac{k}{2}}}{2^{k-1}} \sum_{n=1}^{\infty} \frac{2^{\nu(n)}}{n^{\frac{k}{2}}} \prod_{i=1}^{k}\left(\log \frac{q}{8 n \pi}+\gamma-a_{i} \pi\right)+O_{\varepsilon}\left(k^{A k} q^{\frac{k}{2}-\delta_{k}+\varepsilon}\right)
$$

where $a_{i}=-\frac{1}{2}$ if $i \in \Upsilon$ and $a_{i}=\frac{1}{2}$ otherwise. Indeed, if $\boldsymbol{\alpha}$ and $\boldsymbol{\beta}$ satisfy the hypothesis of Theorem 4, then by contour integration the main term on the right hand side of (2.2) can be 
rewritten as

$$
\begin{aligned}
\sum_{\left\{\alpha_{i}^{\prime}, \beta_{i}^{\prime}\right\}=\left\{\alpha_{i}, \beta_{i}\right\}} \mathscr{M}_{\boldsymbol{\alpha}^{*}, \boldsymbol{\beta}^{*}}= & \frac{q^{\frac{k}{2}-1}}{2^{k-1}} \frac{1}{(2 \pi i)^{k}} \oint_{\left|s_{1}\right|=\frac{1}{4}} \ldots \oint_{\left|s_{k}\right|=\frac{1}{4}} \frac{\zeta\left(\frac{k}{2}+\sum_{i=1}^{k}\left(s_{i}-\alpha_{i}-\beta_{i}\right)\right) \zeta\left(\frac{k}{2}+\sum_{i=1}^{k} s_{i}\right)}{\zeta\left(k+\sum_{i=1}^{k}\left(2 s_{i}-\alpha_{i}-\beta_{i}\right)\right)} \\
& \times \prod_{i=1}^{k} \frac{\Gamma_{i}\left(\frac{1}{4}+\frac{s-\alpha_{i}-\beta_{i}}{2}\right)}{\Gamma_{i}\left(\frac{1}{4}-\frac{s-\alpha_{i}-\beta_{i}}{2}\right)}\left(\frac{q}{\pi}\right)^{s_{i}-\alpha_{i}-\beta_{i}} \zeta\left(1+s_{i}-\alpha_{i}\right) \zeta\left(1+s_{i}-\beta_{i}\right) d s_{i},
\end{aligned}
$$

where the circles are integrated counter-clockwise . Thus, taking the limit for $\boldsymbol{\alpha}, \boldsymbol{\beta} \rightarrow 0$ and expanding $\zeta(s)^{2} / \zeta(2 s)$ as a Dirichlet series (see [Tit], (1.2.8)), we obtain

$$
\begin{aligned}
\sum_{\left\{\alpha_{i}^{\prime}, \beta_{i}^{\prime}\right\}=\left\{\alpha_{i}, \beta_{i}\right\}} \mathscr{M}_{\boldsymbol{\alpha}^{\prime}, \boldsymbol{\beta}^{\prime}} & =\frac{q^{\frac{k}{2}-1}}{2^{k-1}} \sum_{n=1}^{\infty} \frac{2^{\nu(n)}}{n^{\frac{k}{2}}} \prod_{i=1}^{k} \frac{1}{2 \pi i} \oint_{\left|s_{i}\right|=\frac{1}{4}} \frac{\Gamma_{i}\left(\frac{1}{4}+\frac{s_{i}}{2}\right)}{\Gamma_{i}\left(\frac{1}{4}-\frac{s_{i}}{2}\right)}\left(\frac{q}{n \pi}\right)^{s_{i}} \zeta\left(1+s_{i}\right)^{2} d s_{i} \\
& =\frac{q^{\frac{k}{2}-1}}{2^{k-1}} \sum_{n=1}^{\infty} \frac{2^{\nu(n)}}{n^{\frac{k}{2}}} \prod_{i=1}^{k}\left(\log \frac{q}{8 n \pi}+\gamma-a_{i} \pi\right),
\end{aligned}
$$

by the residue theorem. Equation (2.4) then follows.

To prove Theorem 1 we observe that by (2.4) we have (remember that if $|\Upsilon|$ is odd then $\left.M_{\Upsilon, k}=0\right)$

$$
\begin{aligned}
\sum_{a=1}^{q-1}\left(D_{\cos ; 0,0}\left(\frac{1}{2}, \frac{a}{q}\right)\right. & \left.+D_{\sin ; 0,0}\left(\frac{1}{2}, \frac{a}{q}\right)\right)^{k}=\sum_{r=0}^{k} \frac{k !}{r !(k-r) !} \frac{1}{\varphi(q)} \sum_{a=1}^{q-1} D_{\cos ; 0,0}\left(\frac{1}{2}, \frac{a}{q}\right)^{k-r} D_{\sin ; 0,0}\left(\frac{1}{2}, \frac{a}{q}\right)^{r} \\
& =\frac{q^{\frac{k}{2}}}{2^{k-1}} \sum_{n=1}^{\infty} \frac{2^{\nu(n)}}{n^{\frac{k}{2}}} \sum_{\substack{r=0, r \text { even }}} \frac{k !}{r !(k-r) !}\left(\log \frac{q}{8 n \pi}+\gamma-\frac{\pi}{2}\right)^{k-r}\left(\log \frac{q}{8 n \pi}+\gamma+\frac{\pi}{2}\right)^{r}+\mathcal{E}_{2} \\
& =\frac{q^{\frac{k}{2}}}{2^{k}} \sum_{n=1}^{\infty} \frac{2^{\nu(n)}}{n^{\frac{k}{2}}}\left(\left(2 \log \frac{q}{8 n \pi}+2 \gamma\right)^{k}+(-\pi)^{k}\right)+\mathcal{E}_{2}
\end{aligned}
$$

for some $\mathcal{E}_{2} \ll_{\epsilon} k^{A k} q^{\frac{k}{2}-\delta_{k}+\varepsilon}$. Thus, using (1.9) one obtains Theorem 1. One easily verifies that as $q \rightarrow \infty$

$$
\sum_{n=1}^{\infty} \frac{2^{\nu(n)}}{n^{\frac{k}{2}}}\left(\left(\log \frac{q}{8 n \pi}+\gamma\right)^{k}+(-\pi)^{k}\right) \sim \frac{\zeta\left(\frac{k}{2}\right)^{2}}{\zeta(k)}\left(\log \frac{q}{8 \pi}+\gamma\right)^{k}
$$

uniformly in $k \geq 3$. If $k=o\left(\frac{\log q}{\log \log q}\right)$ the error term $\mathcal{E}_{2}$ is smaller than the above main term and so Corollary 1 follows on this range. 
Now assume $k \gg \frac{\log q}{\log \log q}$. First, we observe that by (1.12) for $a \neq 1$ we have

$$
|M(a, q)| \leq(q / \eta)^{\frac{1}{2}} \log q
$$

for any fixed $1<\eta<2$ and $q$ sufficiently large. Indeed, this is obvious if $a=-1$ whereas if $a \neq \pm 1$ then $\max _{j} b_{j} \leq(q-1) / 2$ and so the above bound follows since $b_{1} \cdots b_{\kappa} \leq q$. Furthermore, from the second moment estimate $\sum_{a=1}^{q}|M(a, q)|^{2} \ll(\log q)^{4}$ it follows that for every $C>0$ there are at most $O\left(q(\log q)^{4} / C^{2}\right)$ values of $a$ in $1<a \leq q$ such that $|M(a, q)| \geq C$. Thus, by (2.6) we have

$$
\begin{aligned}
\sum_{a=2}^{q} M(a, q)^{k} & \leq \sum_{\substack{2 \leq a \leq q,|M(a, q)|<C}}^{q} M(a, q)^{k}+\sum_{\substack{2 \leq a \leq q,|M(a, q)| \geq C}}^{q} M(a, q)^{k} \\
& \ll C^{k} q+\frac{q^{\frac{k}{2}+1}(\log q)^{k+4}}{\eta^{\frac{k}{2}} C^{2}} \ll \frac{q^{\frac{k}{2}+\frac{2}{k+2}}}{\eta^{\frac{k}{2}}}(\log q)^{k+2}
\end{aligned}
$$

for $C=\eta^{-\frac{1}{2}} q^{\frac{1}{2}-\frac{1}{k+2}} \log q$. Note that if $k \gg \frac{\log q}{\log \log q}$, then the error term is $\ll q^{\frac{k}{2}} \eta^{-\frac{k}{4}}(\log q)^{k}=$ $o\left(q^{\frac{k}{2}}(\log (q / 8 \pi)+\gamma)^{k}\right)$ as $q \rightarrow \infty$, uniformly in $k$. Finally, we have (cf. [H-B81])

$$
M(1, q)=q^{\frac{1}{2}}(\log (q / 8 \pi)+\gamma)+2 \zeta\left(\frac{1}{2}\right)^{2}+O\left(q^{-\frac{1}{2}}\right)
$$

so that

$$
M(1, q)^{k}=q^{\frac{k}{2}}(\log (q / 8 \pi)+\gamma)^{k} \exp \left(2 \zeta\left(\frac{1}{2}\right)^{2} \frac{k}{q^{1 / 2} \log q}(1+O(1 / \log q))\right)
$$

for $q$ large enough. Thus, if $\frac{\log q}{\log \log q} \ll k=o\left(q^{1 / 2} \log q\right)$ we have

$$
M_{k}(q)=q^{-k / 2} \sum_{a=1}^{q} M(a, q)^{k} \sim(\log (q / 8 \pi)+\gamma)^{k} \sim \frac{\zeta\left(\frac{k}{2}\right)^{2}}{\zeta(k)}(\log (q / 8 \pi)+\gamma)^{k}
$$

as $q \rightarrow \infty$, whereas this asymptotic is false if $k \gg q^{1 / 2} \log q$. This concludes the proof of Corollary 1 .

The proof of Theorem 2 is analogous to those of Theorem 1 and Corollary 1, with the difference that in this case we use (11.8) rather than (1.9). Indeed for some $\mathcal{E}_{1} \ll_{\epsilon} k^{A k} q^{\frac{k}{2}-\delta_{k}+\varepsilon}$ 
we have

$$
\begin{aligned}
& \sum_{a=1}^{q-1} D_{0,0}\left(\frac{1}{2}, \frac{a}{q}\right)^{k}=\sum_{r=0}^{k} \frac{k !}{r !(k-r) !} \sum_{a=1}^{q-1} D_{\cos ; 0,0}\left(\frac{1}{2}, \frac{a}{q}\right)^{k-r} i^{r} D_{\sin ; 0,0}\left(\frac{1}{2}, \frac{a}{q}\right)^{r} \\
& =\frac{q^{\frac{k}{2}}}{2^{k-1}} \sum_{n=1}^{\infty} \frac{2^{\nu(n)}}{n^{\frac{k}{2}}} \sum_{\substack{r=0, r \text { even }}}^{k} \frac{k !}{r !(k-r) !}\left(\log \frac{q}{8 n \pi}+\gamma-\frac{\pi}{2}\right)^{k-r} i^{r}\left(\log \frac{q}{8 n \pi}+\gamma+\frac{\pi}{2}\right)^{r}+\mathcal{E}_{1} \\
& =\frac{q^{\frac{k}{2}}}{2^{k}} \sum_{n=1}^{\infty} \frac{2^{\nu(n)}}{n^{\frac{k}{2}}}\left(\left((1+i)\left(\log \frac{q}{8 n \pi}+\gamma\right)-(1-i) \frac{\pi}{2}\right)^{k}+\left((1-i)\left(\log \frac{q}{8 n \pi}+\gamma\right)-(1+i) \frac{\pi}{2}\right)^{k}\right)+\mathcal{E}_{1} \\
& =(q / 2)^{\frac{k}{2}} 2 \Re\left(\sum_{n=1}^{\infty} \frac{2^{\nu(n)}}{n^{\frac{k}{2}}}\left(e^{\frac{\pi i}{4}}\left(\log \frac{q}{8 n \pi}+\gamma\right)-e^{-\frac{\pi i}{4} \frac{\pi}{2}}\right)^{k}\right)+\mathcal{E}_{1}, \\
& \sim q^{\frac{k}{2}} 2^{1-\frac{k}{2}} \frac{\zeta(k / 2)^{2}}{\zeta(k)} \Re\left(\left(e^{\frac{\pi i}{4}}\left(\log \frac{q}{8 \pi}+\gamma\right)-e^{\left.-\frac{\pi i}{4} \frac{\pi}{2}\right)^{k}}\right)\right.
\end{aligned}
$$

as $q \rightarrow \infty$ with $3 \leq k=o\left(\frac{\log q}{\log \log q}\right)$. One then obtains Theorem 2 on the range $3 \leq k=$ $o\left(q^{\frac{1}{2}} \log q\right)$ by proceeding as in the proof of Corollary 1 .

\subsection{Proof of Theorem 3}

We compute the moments of $f_{r,+}$ only, the case of $f_{r,-}$ being analogous (using (2.8) instead of $(2.7))$.

We start by noticing that Corollary 11 of [Bet16] gives

$$
\begin{aligned}
& D_{\cos ; 0,0}\left(\frac{1}{2}, \frac{a}{q}\right)=\frac{1}{2} \sum_{j=1}^{\kappa} b_{j}^{\frac{1}{2}}\left(\log \frac{b_{j}}{8 \pi}+\gamma-\frac{\pi}{2}\right)+O(\log q), \\
& D_{\sin ; 0,0}\left(\frac{1}{2}, \frac{a}{q}\right)=\frac{1}{2} \sum_{j=1}^{\kappa}(-1)^{j} b_{j}^{\frac{1}{2}}\left(\log \frac{b_{j}}{8 \pi}+\gamma+\frac{\pi}{2}\right)+O(\log q),
\end{aligned}
$$

where $\left[0 ; b_{1}, \ldots b_{\kappa}, 1\right]$ is the continued fraction expansion of $a / q$. Moreover, since $b_{1} \cdots b_{j} \asymp q$, then if one among $b_{1}, \ldots, b_{j}$, say $b_{j^{*}}$, satisfies $b_{j^{*}}>q /(\log q)^{100}$ (and thus in particular $\log b_{j *}=$ $\log q+O(\log \log q))$, then $b_{j} \ll(\log q)^{100}$ for $j \neq j^{*}$. In particular, if $\max _{j} b_{j}>q /(\log q)^{100}$ 
and $1 \leq r=o(\log q / \log \log q)$, then

$$
\begin{aligned}
f_{r,+}\left(\frac{a}{q}\right) & =\sum_{j=1}^{\kappa} b_{j}^{\frac{r}{2}}=\max _{j=1, \ldots \kappa} b_{j}^{\frac{r}{2}}+O\left((\log q)^{50 r+1}\right)=\frac{1}{(\log q)^{r}}\left(\max _{j=1, \ldots \kappa} b_{j}^{\frac{1}{2}} \log q\right)^{r}+O\left((\log q)^{50 r+1}\right) \\
& =\frac{1}{(\log q)^{r}}\left(\left(\max _{j=1, \ldots \kappa} b_{j}^{\frac{1}{2}}\left(\log \frac{b_{j}}{8 \pi}+\gamma-\frac{\pi}{2}\right)\right)(1+O(\log \log q / \log q))\right)^{r}+O\left((\log q)^{50 r+1}\right) \\
& =\frac{2^{r}}{(\log q)^{r}} D_{\cos }\left(\frac{1}{2}, \frac{a}{q}\right)^{r}(1+O(r \log \log q / \log q)) .
\end{aligned}
$$

Moreover, from (2.7) it follows easily that

$$
\sum_{j=1}^{\kappa} b_{j}^{\frac{1}{2}} \leq D_{\cos ; 0,0}\left(\frac{1}{2}, \frac{a}{q}\right)+B \log q
$$

for all $a / q$ and some $B>0$. In particular, if $\max _{j} b_{j} \leq q /(\log q)^{100}$ and $q$ large enough, then

$$
\begin{aligned}
f_{r,+}\left(\frac{a}{q}\right)^{k} & \leq \frac{q^{\frac{k}{2}(r-1)}}{(\log q)^{50 k(r-1)}}\left(\sum_{j=1}^{\kappa} b_{j}^{\frac{1}{2}}\right)^{k} \leq \frac{q^{\frac{k}{2}(r-1)}}{(\log q)^{50 k(r-1)}}\left(D_{\cos ; 0,0}\left(\frac{1}{2}, \frac{a}{q}\right)+B \log q\right)^{k} \\
& \ll \frac{q^{\frac{k r}{2}-1}}{(\log q)^{50 k(r-1)+47(k-2)}}\left(D_{\cos ; 0,0}\left(\frac{1}{2}, \frac{a}{q}\right)+B \log q\right)^{2}
\end{aligned}
$$

for $k \geq 2$, since $\max _{j} b_{j} \leq q /(\log q)^{100}$ implies $\left|D_{\cos }\left(\frac{1}{2}, \frac{a}{q}\right)\right|+B \log q \leq q^{\frac{1}{2}} /(\log q)^{48}$ for $q$ large enough. Now, we have

$$
\sum_{a=1}^{q} f_{r,+}\left(\frac{a}{q}\right)^{k}=\sum_{\substack{1 \leq a<q, \max _{j} b_{j}>q /(\log q)^{100}}} f_{r,+}\left(\frac{a}{q}\right)^{k}+\sum_{\substack{1 \leq a<q, \max _{j} b_{j} \leq q /(\log q)^{100}}} f_{r,+}\left(\frac{a}{q}\right)^{k}
$$

By (2.10) the second summand is bounded by

$$
\begin{aligned}
\sum_{\substack{1 \leq a<q, \max _{j} b_{j} \leq q /(\log q)^{100}}} f_{r,+}(a / q)^{k} & \ll \frac{q^{\frac{k r}{2}-1}}{(\log q)^{50 k(r-1)+48(k-2)}} \sum_{1 \leq a<q}\left(D_{\cos }\left(\frac{1}{2}, \frac{a}{q}\right)+B \log q\right)^{2} \\
& \ll \frac{q^{\frac{k r}{2}}}{(\log q)^{50 k(r-1)+48(k-2)-4}} \ll \frac{q^{k r / 2}}{\log q}
\end{aligned}
$$

for $k r \geq 3$ (if $k=1$ one needs to modify slightly the argument, but the final bound still 
holds). By (2.9) the first summand of (2.11) can be written as

$$
\begin{aligned}
& \sum_{\substack{1 \leq a<q, b_{j}>q /(\log q)^{100}}}^{q} \frac{\left(2 D_{\cos ; 0,0}\left(\frac{1}{2}, \frac{a}{q}\right)\right)^{k r}}{(\log q)^{k r}}(1+O(k r \log \log q / \log q)) \\
= & \sum_{1 \leq a<q} \frac{\left(2 D_{\cos ; 0,0}\left(\frac{1}{2}, \frac{a}{q}\right)\right)^{k r}}{(\log q)^{k r}}(1+O(k r \log \log q / \log q))+O\left(q^{k r / 2} / \log q\right) \\
= & 2 \frac{\zeta(k r)^{2}}{\zeta\left(\frac{k r}{2}\right)} q^{\frac{k r}{2}}(1+O(k r \log \log q / \log q))
\end{aligned}
$$

by (2.4) for $3 \leq r k=o\left(\frac{\log q}{\log \log q}\right)$ and where one can complete the sum by proceeding as in the previous computation. Theorem 3 then follows.

\section{The Estermann function and bounds for sums of Kloost- erman sums}

In this Section we give some results for the Estermann function and for the periodic zetafunction which will be needed in the proof of Theorem 4. In particular, in Section 3.1 we give the functional equation for both these functions, whereas in Section 3.2 we give a version of the approximate functional equation for the Estermann function. Finally, in Section 3.3 we give some estimates for products of the Estermann function and the periodic zeta-function, using the bounds of [DI] for sums of Kloosterman sums.

\subsection{The functional equations}

We start by giving the functional equation for the Estermann function.

Lemma 2. For $(a, q)=1, q>0$ and $\alpha \in \mathbb{C}, D_{\alpha, \beta}\left(s, \frac{a}{q}\right)-q^{1-\alpha-\beta-2 s} \zeta(s+\alpha) \zeta(s+\beta)$ can be extended to an entire function of $s$. Moreover, $D_{\alpha, \beta}\left(s, \frac{a}{q}\right)$ satisfies the functional equation

$$
\begin{aligned}
D_{\alpha, \beta}\left(s, \frac{a}{q}\right)= & -\frac{2}{q}\left(\frac{q}{2 \pi}\right)^{2-2 s-\alpha-\beta} \Gamma(1-s-\alpha) \Gamma(1-s-\beta) \times \\
& \times\left(\cos \left(\frac{\pi}{2}(2 s+\alpha+\beta)\right) D_{-\alpha,-\beta}\left(1-s,-\frac{\bar{a}}{q}\right)-\cos \frac{\pi(\alpha-\beta)}{2} D_{\alpha, \beta}\left(1-s, \frac{\bar{a}}{q}\right)\right),
\end{aligned}
$$

where, here and in the following, $\bar{a}$ denotes the multiplicative inverse of a modulo the denominator $q$. 
Proof. The lemma follows easily from the analytic continuation and the functional equation for the Hurwitz zeta function $\zeta(s, x)$ (see Apo, Theorem 12.8) and from the decomposition

$$
D_{\alpha, \beta}\left(s, \frac{h}{k}\right)=q^{-\alpha-\beta-2 s} \sum_{m, n=1}^{q} \mathrm{e}\left(\frac{m n a}{q}\right) \zeta\left(s+\alpha, \frac{m}{q}\right) \zeta\left(s+\beta, \frac{n}{q}\right) .
$$

Corollary 3. Let

$$
\begin{aligned}
& \Lambda_{c ; \alpha, \beta}\left(s, \frac{a}{q}\right):=\Gamma\left(\frac{s+\alpha}{2}\right) \Gamma\left(\frac{s+\beta}{2}\right)\left(\frac{q}{\pi}\right)^{s+\frac{\alpha+\beta}{2}} D_{c ; \alpha, \beta}\left(s, \frac{a}{q}\right), \\
& \Lambda_{s ; \alpha, \beta}\left(s, \frac{a}{q}\right):=\Gamma\left(\frac{1+s+\alpha}{2}\right) \Gamma\left(\frac{1+s+\beta}{2}\right)\left(\frac{q}{\pi}\right)^{s+\frac{\alpha+\beta}{2}} D_{s ; \alpha, \beta}\left(s, \frac{a}{q}\right) .
\end{aligned}
$$

Then, we have the functional equations

$$
\Lambda_{c ; \alpha, \beta}\left(s, \frac{a}{q}\right)=\Lambda_{c ;-\alpha,-\beta}\left(1-s, \frac{\bar{a}}{q}\right), \quad \Lambda_{s ; \alpha, \beta}\left(s, \frac{a}{q}\right)=\Lambda_{s ;-\alpha,-\beta}\left(1-s, \frac{\bar{a}}{q}\right) .
$$

Proof. These functional equations follow from (3.1), using the reflection and the duplication formulas for the $\Gamma$-function.

We also need the basic properties of the periodic zeta-function which, for $x \in \mathbb{R}$ and $\Re(s)>1$, is defined as

$$
F(s, x):=\sum_{n=1}^{\infty} \frac{\mathrm{e}(n x)}{n^{s}}
$$

Notice that if $x=1$, then $F(s, x)=\zeta(s)$.

Lemma 4. Let $h, l \in \mathbb{Z}$ with $(h, \ell)=1$ and $\ell>0$, then $F(s, h / \ell)$ extends to an entire function of $s$ with the exception of a simple pole at $s=1$ if $\ell=1$. Moreover, $F(s, x)$ satisfies the functional equation

$$
F(1-s, h / \ell)=\ell^{s-1} \sum_{b=1}^{\ell} \mathrm{e}(h b / \ell) \frac{\Gamma(s)}{(2 \pi)^{s}}\left(e^{-\frac{\pi i s}{2}} F(s, b / \ell)+e^{\frac{\pi i s}{2}} F(s,-b / \ell)\right) .
$$

Finally, for $\ell \nmid h$ we have

$$
F(0, h / \ell)=-\frac{1}{2}+\frac{i}{2} \cot (\pi h / \ell)
$$


Proof. If $\Re(s)<0$, then splitting the series defining $F(s, h / \ell)$ according to the congruence class of $n$ modulo $\ell$ we obtain

$$
F(1-s, h / \ell)=\ell^{s-1} \sum_{b=1}^{\ell} \mathrm{e}(h b / \ell) \zeta(1-s, b / \ell),
$$

where $\zeta(s, x)$ the Hurwitz zeta-function. Thus the analytic continuation of $F(s, h / \ell)$ follows from that of $\zeta(s, x)$ since $\zeta(s, x)$ is holomorphic on $\mathbb{C}$ with the exception of a simple pole of residue 1 at $s=1$. The functional equation (3.4) follows immediately from the identity

$$
\zeta(1-s, x)=(2 \pi)^{-s} \Gamma(s)\left(e^{-\frac{\pi i s}{2}} F(s, x)+e^{\frac{\pi i s}{2}} F(s,-x)\right)
$$

(see Apo, Theorem 12.6), whereas (3.5) follows from (3.6), the Laurent expansion for $\zeta(s, x)$ (cf. [WW], p. 271)

$$
\zeta(s, x)=\frac{1}{s-1}-\psi(x)+O(|s-1|)
$$

where $\psi(x)$ is the digamma function, and the identity $\psi(1-x)-\psi(x)=\pi \cot (\pi x)$.

\subsection{The approximate functional equation}

Next, we give an approximate functional equation allowing us to express a product of $k$ Estermann functions as a sum of total length about $q^{\frac{k}{2}}$. In this Lemma and in the rest of the paper we will often omit to indicate the dependencies on $\Upsilon$.

Lemma 5. Let $k \geq 1$ and $\Upsilon \subseteq\{1, \ldots, k\}$. Let $G_{\boldsymbol{\alpha}, \boldsymbol{\beta}}(s)$ be an entire function satisfying $G_{\boldsymbol{\alpha}, \boldsymbol{\beta}}(-s)=G_{-\boldsymbol{\alpha},-\boldsymbol{\beta}}(s), G_{\boldsymbol{\alpha}, \boldsymbol{\beta}}(0)=1$ and $G_{\boldsymbol{\alpha}, \boldsymbol{\beta}}\left(\frac{1}{2}-\alpha_{i}\right)=G_{\boldsymbol{\alpha}, \boldsymbol{\beta}}\left(\frac{1}{2}-\beta_{i}\right)=0$ for $i=1, \ldots, k$ and decaying faster than any power of $s$ on vertical strips. Let

$$
\begin{aligned}
g_{\boldsymbol{\alpha}, \boldsymbol{\beta}}(s) & :=\pi^{-k s} \prod_{j=1}^{k} \frac{\Gamma_{i}\left(\frac{\frac{1}{2}+s+\alpha_{i}}{2}\right) \Gamma_{i}\left(\frac{\frac{1}{2}+s+\beta_{i}}{2}\right)}{\Gamma_{i}\left(\frac{\frac{1}{2}+\alpha_{i}}{2}\right) \Gamma_{i}\left(\frac{\frac{1}{2}+\beta_{i}}{2}\right)} \\
X_{\boldsymbol{\alpha}, \boldsymbol{\beta}} & :=\prod_{j=1}^{k} \frac{\Gamma_{i}\left(\frac{\frac{1}{2}-\alpha_{i}}{2}\right) \Gamma_{i}\left(\frac{\frac{1}{2}-\beta_{i}}{2}\right)}{\Gamma_{i}\left(\frac{\frac{1}{2}+\alpha_{i}}{2}\right) \Gamma_{i}\left(\frac{\frac{1}{2}+\beta_{i}}{2}\right)}\left(\frac{q}{\pi}\right)^{-\alpha_{i}-\beta_{i}}
\end{aligned}
$$

and for any $c_{s}>0$ let

$$
V_{\boldsymbol{\alpha}, \boldsymbol{\beta}}(x):=\frac{1}{2 \pi i} \int_{\left(c_{s}\right)} G_{\boldsymbol{\alpha}, \boldsymbol{\beta}}(s) g_{\boldsymbol{\alpha}, \boldsymbol{\beta}}(s) x^{-s} \frac{d s}{s},
$$


where, as usual, $\int_{(c)} \cdot d s$ indicates that the integral is taken along the vertical line from $c-i \infty$ to $c+i \infty$. Then for $a, q \in \mathbb{Z}$, with $q>1$ and $(a, q)=1$ we have

$$
\prod_{i=1}^{k} D_{i ; \alpha_{i}, \beta_{i}}\left(\frac{1}{2}, \frac{a}{q}\right)=S_{\boldsymbol{\alpha}, \boldsymbol{\beta}}(a, q)+X_{\boldsymbol{\alpha}, \boldsymbol{\beta}} S_{-\boldsymbol{\alpha},-\boldsymbol{\beta}}(\bar{a}, q)
$$

where $\bar{a}$ is the inverse of a modulo $q$ and

$$
\begin{aligned}
S_{\boldsymbol{\alpha}, \boldsymbol{\beta}}(a, q):= & \frac{i^{-|\Upsilon|}}{2^{k}} \sum_{\substack{\epsilon=\left( \pm_{1} 1, \ldots, \pm_{k} 1\right) \in\{ \pm 1\}^{k} \\
n_{1}, \ldots, n_{k} \geq 1}} \rho_{\Upsilon}(\epsilon) \frac{\tau_{\alpha_{1}, \beta_{1}}\left(n_{1}\right) \cdots \tau_{\alpha_{k}, \beta_{k}}\left(n_{k}\right)}{\left(n_{1} \cdots n_{k}\right)^{\frac{1}{2}}} \times \\
& \times \mathrm{e}\left(\frac{a\left( \pm_{1} n_{1} \pm_{2} \cdots \pm_{k} n_{k}\right)}{q}\right) V_{\boldsymbol{\alpha}, \boldsymbol{\beta}}\left(\frac{n_{1} \cdots n_{k}}{q^{k}}\right),
\end{aligned}
$$

with $\rho_{\Upsilon}(\epsilon):=\prod_{i \in \Upsilon}\left( \pm_{i} 1\right)$.

Proof. By contour integration and the functional equation, we have

$$
\begin{aligned}
\prod_{i=1}^{k} \Lambda_{i ; \alpha_{i}, \beta_{i}}\left(\frac{1}{2}, \frac{a}{q}\right)= & \frac{1}{2 \pi i}\left(\int_{(2)}-\int_{(-2)}\right) \prod_{i=1}^{k} \Lambda_{i ; \alpha_{i}, \beta_{i}}\left(\frac{1}{2}+s, \frac{a}{q}\right) \cdot G_{\boldsymbol{\alpha}, \boldsymbol{\beta}}(s) \frac{d s}{s} \\
= & \frac{1}{2 \pi i} \int_{(2)} \prod_{i=1}^{k} \Lambda_{i ; \alpha_{i}, \beta_{i}}\left(\frac{1}{2}+s, \frac{a}{q}\right) \cdot G_{\boldsymbol{\alpha}, \boldsymbol{\beta}}(s) \frac{d s}{s} \\
& +\frac{1}{2 \pi i} \int_{(2)} \prod_{i=1}^{k} \Lambda_{i ;-\alpha_{i},-\beta_{i}}\left(\frac{1}{2}, \frac{\bar{a}}{q}\right) \cdot G_{-\boldsymbol{\alpha},-\boldsymbol{\beta}}(s) \frac{d s}{s} .
\end{aligned}
$$

Now, expanding the Estermann functions into their Dirichlet series, we see that

$$
\begin{aligned}
\frac{1}{2 \pi i} \int_{(2)} \prod_{i=1}^{k} \frac{\Lambda_{i ; \alpha_{i}, \beta_{i}}\left(\frac{1}{2}+s, \frac{a}{q}\right)}{\Gamma_{i}\left(\frac{\frac{1}{2}+\alpha_{i}}{2}\right) \Gamma_{i}\left(\frac{\frac{1}{2}+\beta_{i}}{2}\right)\left(\frac{q}{\pi}\right)^{\frac{1}{2}+\frac{\alpha_{i}+\beta_{i}}{2}}} \cdot G_{\boldsymbol{\alpha}, \boldsymbol{\beta}}(s) \frac{d s}{s} \\
=\frac{i^{-|\Upsilon|}}{2^{k}} \sum_{n_{1}, \ldots, n_{k} \in \mathbb{Z} \backslash\{0\}} \operatorname{sgn}\left(\prod_{i \in \Upsilon} n_{i}\right) \frac{\tau_{\alpha_{1}, \beta_{1}}\left(\left|n_{1}\right|\right) \cdots \tau_{\alpha_{k}, \beta_{k}}\left(\left|n_{k}\right|\right)}{\left|n_{1} \cdots n_{k}\right|^{\frac{1}{2}}} \mathrm{e}\left(\frac{a\left(n_{1}+\cdots+n_{k}\right)}{q}\right) \\
\quad \times \frac{1}{2 \pi i} \int_{(2)} G_{\boldsymbol{\alpha}, \boldsymbol{\beta}}(s) g_{\boldsymbol{\alpha}, \boldsymbol{\beta}}(s)\left(\frac{\left|n_{1} \cdots n_{k}\right|}{q^{k}}\right)^{-s} \frac{d s}{s}
\end{aligned}
$$

and the lemma follows. 


\subsection{Estimates for the Estermann function}

In this section we give two bounds for certain averages of products the Estermann function and the periodic zeta-function. Both bounds depend on estimates for Kloosterman sums, more specifically on Weil's bound and on (a minor modification of) a bound by Deshouilliers and Iwaniec [DI]. We recall that the classical Kloosterman sum is defined as

$$
S(m, n ; \ell):=\sum_{c(\bmod \ell)}^{*} \mathrm{e}\left(\frac{m c+n \bar{c}}{\ell}\right)
$$

for any $c, m, n \in \mathbb{Z}, c \geq 1$, where $\sum^{*}$ indicates that the sum is over $c(\bmod \ell)$ such that $(c, \ell)=1$. Also, we recall that Weil's bound gives $S(m, n ; \ell) \ll d(\ell)(m, n, \ell)^{\frac{1}{2}} \ell^{\frac{1}{2}}$. Using this bound we obtain the following Lemma.

Lemma 6. Let $r>0,0<\delta<1, C \geq 2, \eta_{0} \neq 0$ and $\left(\eta_{1}, \ldots, \eta_{r}\right) \in\{ \pm 1\}^{r}$. Let $|a| \leq 2 C \delta$, $|b| \leq C \delta$ and $\left|a_{j}\right|,\left|b_{j}\right|<\delta$ for $j=1, \ldots, r$. Then, for some $A>0$ we have

$$
\begin{aligned}
\sum_{\ell \geq 1} \frac{1}{\ell C+a} \sum_{h(\bmod \ell)}^{*} F\left(1+C\left(s-\frac{1}{2}\right)+b, \frac{\eta_{0} h}{\ell}\right) \prod_{j=1}^{r} D_{a_{j}, b_{j}}\left(\frac{1}{2}, \frac{\eta_{j} h}{\ell}\right) & \\
& \ll_{\delta}(A C / \delta)^{A(r+C)}(1+|s|)^{A(r+C)}
\end{aligned}
$$

in the strip

$$
-\frac{1}{2}+\frac{r+\frac{3}{2}}{C+r-\frac{1}{2}}+8 \delta<\Re(s)<\frac{1}{2}-2 \delta .
$$

Moreover, the left hand side of (3.9) is meromorphic in the half plane $\Re(s)>-\frac{1}{2}+\frac{r+\frac{3}{2}}{C+r-\frac{1}{2}}+8 \delta$ with poles at $s=\frac{1}{2}-a_{j}$ and $s=\frac{1}{2}-b_{j}$ for $j=1, \ldots, r$ and $s=\frac{1}{2}-b / C$ and these poles are simple if $a_{1}, \ldots, a_{r}, b_{1}, \ldots, b_{r}$ and $b / C$ are all distinct.

Proof. For $L \geq 1$, let

$$
\begin{aligned}
H_{L}(s) & :=\sum_{L<\ell \leq 2 L} \frac{1}{\ell^{C+a}} \sum_{h(\bmod \ell)}^{*} F\left(1+C\left(s-\frac{1}{2}\right)+b, \frac{\eta_{0} h}{\ell}\right) \prod_{j=1}^{r} D_{a_{j}, b_{j}}\left(\frac{1}{2}+s, \frac{\eta_{j} h}{\ell}\right), \\
K(s) & :=\prod_{j=1}^{r}\left(s-\frac{1}{2}+a_{j}\right)\left(s-\frac{1}{2}+b_{j}\right) .
\end{aligned}
$$

Notice that if $\ell \neq 1$ and $(h, \ell)=1$ then $F(x, h / \ell)$ is entire and thus so is $H_{L}(s) K(s)$ for all $L \geq 1$. Now, if $\Re(s)=\frac{1}{2}+2 \delta$, then a trivial bound gives

$$
H_{L}(s) K(s) \ll\left(1+|s|^{2 r}\right)(A / \delta)^{2 r+1} L^{-C+2+2 \delta C},
$$


where, here and in the following, $A$ denotes a sufficiently large positive constant, which might change from line to line.

Next, take $\Re(s)=-\frac{1}{2}-2 \delta$. Then, applying the functional equations (3.1) and (3.4) to $D$ and $F$, expanding $D$ and $F$ into their Dirichlet series, and using Stirling's formula in the crude form

$$
\Gamma(\sigma+i t) \ll c^{-1}(1+A|\sigma|)^{|\sigma|}(1+|t|)^{\sigma-\frac{1}{2}} e^{-\frac{\pi}{2}|t|}, \quad \sigma \geq c>0
$$

we see that

$$
\begin{aligned}
H_{L}(s) K(s) \ll & A^{r} C^{A C}(1+|s|)^{A(r+C)} L^{r-1+(5 C+6 r) \delta} \sum_{L<\ell \leq 2 L} \sum_{u=1}^{\ell}\left|F\left(C\left(\frac{1}{2}-s\right)-b, u / \ell\right)\right| \times \\
& \times \sum_{n_{1}, \cdots, n_{r} \in \mathbb{Z}_{\neq 0}} \frac{\left|\tau-a_{1},-b_{1}\left(\left|n_{1}\right|\right) \cdots \tau_{-a_{r},-b_{r}}\left(\left|n_{r}\right|\right)\right|}{\left|n_{1}^{1+2 \delta} \cdots n_{r}^{1+2 \delta}\right|}\left|S\left(\eta_{0} u, n_{1}+\cdots+n_{k} ; \ell\right)\right|,
\end{aligned}
$$

and thus by Weil's bound we obtain

$$
H_{L}(s) K(s) \ll(A / \delta)^{2 r+5} C^{A C}(1+|s|)^{A(r+C)} L^{r+\frac{3}{2}+6(r+C) \delta},
$$

when $\Re(s)=-\frac{1}{2}-2 \delta$. Thus, by (3.10), (3.12) and the Phragmén-Lindelöf principle, if $-\frac{1}{2}-2 \delta \leq \Re(s) \leq \frac{1}{2}+2 \delta$ we have

$$
H_{L}(s) K(s) \ll(A / \delta)^{2 r+5} C^{A C}(1+|s|)^{A(r+C)} L^{r+\frac{3}{2}-\left(C+r-\frac{1}{2}\right)\left(\Re(s)+\frac{1}{2}\right)+5 \delta(r+C)} .
$$

Moreover, if $\left|s-\frac{1}{2}\right|>2 \delta$ then $K(s) \gg \delta^{2 r}$ and thus, if $-\frac{1}{2}-2 \delta \leq \Re(s) \leq \frac{1}{2}-2 \delta$, we have

$$
H_{L}(s) \ll(A / \delta)^{4 r+5} C^{A C}(1+|s|)^{A(r+C)} L^{r+\frac{3}{2}-\left(C+r-\frac{1}{2}\right)\left(\Re(s)+\frac{1}{2}\right)+5 \delta(r+C)} .
$$

It follows that if

$$
-\frac{1}{2}+\frac{r+\frac{3}{2}+6 \delta(r+C)}{C+r-\frac{1}{2}} \leq \Re(s) \leq \frac{1}{2}-2 \delta
$$

then

$$
\begin{aligned}
\sum_{\ell>1} \frac{1}{\ell C+a} \sum_{h(\bmod \ell)}^{*} F\left(1+C\left(s-\frac{1}{2}\right)+b, \frac{\eta_{0} h}{\ell}\right) \prod_{j=1}^{r} D_{a_{j}, b_{j}}\left(\frac{1}{2}+s, \frac{\eta_{j} h}{\ell}\right) & \\
& \ll_{\delta}(A / \delta)^{4 r+6} C^{A C}(1+|s|)^{A(r+C)} .
\end{aligned}
$$

Finally, the contribution of the $\ell=1$ term to the left hand side of (3.9) is

$$
\zeta\left(1+C\left(s-\frac{1}{2}\right)+b\right) \prod_{j=1}^{r} \zeta\left(\frac{1}{2}+s+a_{j}\right) \zeta\left(\frac{1}{2}+s+b_{j}\right) \ll(A / \delta)^{2 r+1} C^{A C}(1+|s|)^{A(r+C)}
$$


when $s$ satisfies (3.13) and thus (3.9) follows. We conclude by remarking that the above computations also give the meromorphicity of the left hand side of (3.9) on $\Re(s) \geq-\frac{1}{2}+$ $\frac{r+\frac{3}{2}+5 \delta(r+C)}{C+r-\frac{1}{2}}$.

We now states a variation of a bound by Deshouilliers-Iwaniec for sums of Kloosterman sums (cf. Theorem 9 and (1.52) of [DI]), which is essentially implicit in [BHM] and [HWW] (cf. Theorem 1.4 of [Wat]).

Lemma 7. Let $W$ be a smooth function supported in $[1,2]$ and satisfying $W^{(i)}(x) \ll C^{i}$ for $i=0,1,2$ and some $C>1$. Let $a_{m}, b_{n} \ll 1$ be sequences of complex numbers supported in $[M, 2 M]$ and $[N, 2 N]$ respectively. Then, for $q \geq 1$ and $\eta \in\{ \pm 1\}$ we have

$$
\sum_{m, n, \ell \geq 1} W(\ell / L) a_{m} b_{n} S(q m, \eta n ; \ell) \ll_{\varepsilon} q^{\vartheta+\varepsilon} C^{4}\left(L^{1+\varepsilon}+q^{\frac{1}{2}}\right) M N,
$$

where $\vartheta=\frac{7}{64}$.

Proof. After splitting the sum over $m$ according to the common factor of $m$ with $q$, one can repeat the arguments used in the proof of Theorem 9 of [DI] but using the multiplicativity of Hecke-eigenvalues (which holds since we are in the case of level 1, for which there are only new-forms) just before applying the spectral large sieve. Kim-Sarnak's bound Kim for Hecke eigenvalues then gives (3.14).

The above argument was carried out in detail (sorting out also the delicate issues arising when considering a level different from 1) in BHM], Theorem 4 . We remark that the factor $(1+C / \sqrt{M N})^{2 \vartheta}$ appearing there can be removed when the level is $D=1$ since Selberg's eigenvalue conjecture is known in the case of $\mathrm{SL}(2, \mathbb{Z})$.

Remark 3. Using the variation of the spectral large sieve given by Blomer and Milićević in Theorem 8 of $[B M]$, one obtains a bound which improves upon (3.14) when the parameters are in certain ranges. It is likely that the use of such bound in combination with (3.14) would lead to a better bound for the error term in Theorem 4. However for simplicity we choose to use (3.14) in all ranges, since this is sufficient for our purposes.

Using Lemma 7 we obtain the following result.

Lemma 8. For $r \geq 1$, let $t_{0}, \cdots, t_{r} \in \mathbb{R},\left(\eta_{1}, \ldots, \eta_{r}\right) \in\{ \pm 1\}^{r}$, and let $\eta_{0} \neq 0$. Furthermore, let $\left|a_{j}\right|,\left|b_{j}\right|<\delta$ for $j=1, \ldots, r$ and some $0<\delta<1$. Finally, let $L>0$ and let $W(x)$ be $a$ function supported on $[1,2]$ with $W^{(i)}(x) \ll 1$ for $i=0,1,2$. Then, if $w \in \mathbb{C}$ and $\sigma \geq 2 \delta$, we have

$$
\mathfrak{S}:=\sum_{\ell \geq 1} \frac{W(\ell / L)}{\ell^{1+w}} \sum_{h(\bmod \ell)}^{*} F\left(1+\sigma+i t_{0}, \frac{\eta_{0} h}{\ell}\right) \prod_{j=1}^{r} D_{a_{j}, b_{j}}\left(-\sigma+i t_{j}, \frac{\eta_{j} h}{\ell}\right)
$$


is bounded by

$$
\mathfrak{S} \ll_{\delta} L^{r(3 \sigma+1)-\Re(w)} \frac{A^{r(\sigma+1)}}{\delta^{2 r}} K_{r}\left(\sigma, w, t_{1}, \ldots, t_{j}\right) \times \begin{cases}\left|\eta_{0}\right|^{\vartheta+\delta} & \text { if } L \geq\left|\eta_{0}\right|^{\frac{1}{2}} \\ \left|\eta_{0}\right|^{\frac{1}{6}+\frac{\vartheta}{3}+\delta} & \text { always }\end{cases}
$$

for some absolute $A>0$ and where

$$
K_{r}\left(s, w, t_{1}, \ldots, t_{j}\right):=(1+\sigma)^{2 r(2 \sigma+1)}(1+|w|)^{4} \prod_{j=1}^{r}\left(1+|s|+\left|t_{j}\right|\right)^{1+4 \sigma}
$$

Proof. Applying the functional equation (3.1), expanding $D$ and $F$ into their Dirichlet series, and using (3.11) we obtain

$$
\mathfrak{S} \ll_{\delta} A^{r}(1+A \sigma)^{2 r(\sigma+\delta+1)}\left(\prod_{j=1}^{r}\left(1+\left|t_{j}\right|\right)^{2(\sigma+\delta)+1}\right)\left|\sum_{\ell \geq 1} \sum_{m \geq 1} \sum_{n \in \mathbb{Z}} \frac{W_{0}(\ell) f_{n}}{m^{1+\sigma+i t_{0}}} S\left(\eta_{0} m, n ; \ell\right)\right|,
$$

where

$$
\begin{aligned}
W_{0}(x) & :=W(x / L) x^{r(2 \sigma+1)-1-w-\sum_{j=1}^{r}\left(t_{j}+a_{j}+b_{j}\right)} \ll(2 L)^{r(3 \sigma+1)-1-\Re(w)}, \\
f_{n} & :=\sum_{\substack{n_{1}, \cdots, n_{r} \in \mathbb{Z}_{\neq 0}, n_{1}+\cdots+n_{r}=n}} \frac{\tau_{-a_{1},-b_{1}}\left(n_{1}\right)}{n_{1}^{1+s-i t_{1}}} \cdots \frac{\tau_{-a_{r},-b_{r}}\left(n_{r}\right)}{n_{r}^{1+s-i t_{r}}} \ll \delta(A / \delta)^{2 r} \frac{1}{|n|^{1+\delta / 2}} .
\end{aligned}
$$

Splitting the sums over $n$ and $m$ into diadic blocks and applying (3.14) one easily gets the bound

$$
\mathfrak{S} \ll_{\delta} L^{r(3 \sigma+1)-\Re(w)} \frac{A^{r(\sigma+1)}}{\delta^{2 r}} K_{r}\left(\sigma, w, t_{1}, \ldots, t_{j}\right)\left|\eta_{0}\right|^{\vartheta+\delta}\left(1+\left|\eta_{0}\right|^{\frac{1}{2}} / L\right),
$$

which gives (3.15) in the case $L \geq\left|\eta_{0}\right|^{\frac{1}{2}}$. Applying Weil's bound rather than (3.14), one obtains

$$
\mathfrak{S} \ll_{\delta} L^{r(3 \sigma+1)-\Re(w)} \frac{A^{r(\sigma+1)}}{\delta^{2 r}} K_{r}\left(\sigma, w, t_{1}, \ldots, t_{j}\right) L^{\frac{1}{2}},
$$

and taking the minimum between (3.16) and (3.17) one gets (3.15) also in the case $L<$ $\left|\eta_{0}\right|^{\frac{1}{2}}$

\section{Some assumptions}

In this section we set up some notation and make some simplifying assumptions, which we will use throughout the rest of the paper. 
First, $q$ will always denote a prime, $k$ an integer greater than 2 , and $\Upsilon$ a subset of $\{1, \ldots, k\}$ with even cardinality. Moreover we shall use the convention that $A$ and $\varepsilon$ denote a sufficiently large and arbitrarily small positive constants on which the implicit bounds are allowed to depend and whose values might change from line to line.

Also, we assume $\boldsymbol{\alpha}=\left(\alpha_{1}, \ldots, \alpha_{k}\right) \in \mathbb{A}_{2 C}^{k}, \boldsymbol{\beta}=\left(\beta_{1}, \ldots, \beta_{k}\right) \in \mathbb{A}_{C / 2}^{k}$ for some constant $C>0$ (with $4 C / \log q \leq 1 / 10$ ), where $\mathbb{A}_{r}$ denotes the annulus $\{s \in \mathbb{C}|C / \log q \leq| s \mid \leq 2 C / \log q\}$. This assumption can then be removed by analytic continuation and the maximum modulus principle, since both the left hand side and, by (2.5), the main term on the right hand side of (2.2) are analytic functions of the shifts in $\left|\alpha_{i}\right|,\left|\beta_{i}\right| \leq 4 C / \log q$. We remark in particular, that with the above assumption, we have $\left|\alpha_{i}\right|,\left|\beta_{i}\right|,\left|\alpha_{i}-\beta_{i}\right| \asymp 1 / \log q$.

Moreover, for the rest of the paper we fix an entire function $G_{\boldsymbol{\alpha}, \boldsymbol{\beta}}(s)$ as follow:

$$
G_{\boldsymbol{\alpha}, \boldsymbol{\beta}}(s):=\frac{Q_{\boldsymbol{\alpha}, \boldsymbol{\beta}}(s)}{Q_{\boldsymbol{\alpha}, \boldsymbol{\beta}}(0)} \frac{\xi\left(\frac{1}{2}+s\right)}{\xi\left(\frac{1}{2}\right)},
$$

where $\xi(s):=\frac{1}{2} s(s-1) \pi^{-\pi / 2} \Gamma\left(\frac{1}{2} s\right) \zeta(s)$ is the Riemann $\xi$-function and

$$
Q_{\boldsymbol{\alpha}, \boldsymbol{\beta}}(s):=\prod_{i=1}^{k}\left(s^{2}-\left(\alpha_{i}-\beta_{i}\right)^{2}\right) .
$$

By the functional equation for the Riemann zeta-function we have $G_{\boldsymbol{\alpha}, \boldsymbol{\beta}}(-s)=G_{-\boldsymbol{\alpha},-\boldsymbol{\beta}}(s)$ and so $G_{\boldsymbol{\alpha}, \boldsymbol{\beta}}(s)$ satisfies the hypothesis of Lemma 5. Moreover, using Stirling's formula (3.11) we also obtain

$$
G_{\boldsymbol{\alpha}, \boldsymbol{\beta}}(s) \ll(\log q)^{2 k} e^{-C_{1}|t|}(1+|\sigma|)^{A(|\sigma|+k)},
$$

for all $s=\sigma+i t \in \mathbb{C}$ and some $C_{1}>0$.

Finally, we notice that from the functional equations (3.2), for $i=1, \ldots, k$, we have the convexity bound

$$
D_{i}\left(\frac{1}{2}+\alpha_{i}, \alpha_{i}-\beta_{i}, \frac{a}{q}\right) \ll q^{\frac{1}{2}}(\log q)^{2}
$$

and so trivially $M_{\Upsilon, k} \ll\left(A q^{\frac{1}{2}}(\log q)^{2}\right)^{k}$. Also, from (2.5) it is easy to see that one also has

$$
\sum_{\left\{\alpha_{i}^{\prime}, \beta_{i}^{\prime}\right\}=\left\{\alpha_{i}, \beta_{i}\right\}} \mathscr{M}_{\boldsymbol{\alpha}^{\prime}, \boldsymbol{\beta}^{\prime}} \ll q^{\frac{k}{2}-1}(A \log q)^{k} .
$$

It follows that Theorem 4 is trivial if $k \gg \log q / \log \log q$ since in this case $(A k)^{A k} \gg q^{A / 2}$. Thus, we will assume $k=o(\log q / \log \log q)$. In particular, for $q$ large enough we have $\left|\alpha_{i}\right|,\left|\beta_{i}\right|<1 / \log q<1 /(k \log \log q)<\frac{\varepsilon}{2 k}$ and a fortiori

$$
\left|\alpha_{1}\right|+\cdots+\left|\alpha_{k}\right|+\left|\beta_{1}\right|+\cdots+\left|\beta_{k}\right|<\varepsilon .
$$

Moreover, notice that under these assumptions we also have the inequality $(k / \varepsilon)^{A k} \ll(\log q)^{A k} \ll$ $q^{\varepsilon}$ which we shall often use. 


\section{Dividing into diagonal and off-diagonal terms and structure of the proof}

By the approximate functional equation (3.8) and the orthogonality of additive characters,

we can decompose $M_{\Upsilon, k}$ into diagonal and off-diagonal terms:

$$
M_{\Upsilon, k}:=\frac{1}{\varphi(q)} \sum_{a=1}^{q-1} \prod_{i=1}^{k} D_{i ; \alpha_{i}, \beta_{i}}\left(\frac{1}{2}, \frac{a}{q}\right)=\mathcal{D}_{\boldsymbol{\alpha}, \boldsymbol{\beta}}+X_{\boldsymbol{\alpha}, \boldsymbol{\beta}} \mathcal{D}_{-\boldsymbol{\alpha},-\boldsymbol{\beta}}+\mathcal{O}_{\boldsymbol{\alpha}, \boldsymbol{\beta}}+X_{\boldsymbol{\alpha}, \boldsymbol{\beta}} \mathcal{O}_{-\boldsymbol{\alpha},-\boldsymbol{\beta}}
$$

where

$$
\begin{aligned}
& \mathcal{D}_{\boldsymbol{\alpha}, \boldsymbol{\beta}}:=\frac{i^{|\Upsilon|}}{2^{k}} \sum_{\epsilon \in\{ \pm 1\}^{k}} \rho_{\Upsilon}(\epsilon) \sum_{ \pm_{1} n_{1}+\cdots+ \pm_{k} n_{k}=0} \frac{\tau_{\alpha_{1}, \beta_{1}}\left(n_{1}\right) \cdots \tau_{\alpha_{k}, \beta_{k}}\left(n_{k}\right)}{\left(n_{1} \cdots n_{k}\right)^{\frac{1}{2}}} V_{\boldsymbol{\alpha}, \boldsymbol{\beta}}\left(\frac{n_{1} \cdots n_{k}}{q^{k}}\right), \\
& \mathcal{O}_{\boldsymbol{\alpha}, \boldsymbol{\beta}}:=\frac{i^{|\Upsilon|}}{2^{k}} \sum_{\epsilon \in\{ \pm 1\}^{k}} \rho_{\Upsilon}(\epsilon) \mathcal{O}_{\epsilon, \boldsymbol{\alpha}, \boldsymbol{\beta}}^{\prime} \\
& \mathcal{O}_{\epsilon, \boldsymbol{\alpha}, \boldsymbol{\beta}}^{\prime}:=\sum_{d \mid q} d \frac{\mu\left(\frac{q}{d}\right)}{\varphi(q)} \sum_{\substack{d \mid\left( \pm_{1} n_{1}+\cdots+ \pm_{k} n_{k}\right), \pm_{1} n_{1}+\cdots+ \pm_{k} n_{k} \neq 0}} \frac{\tau_{\alpha_{1}, \beta_{1}}\left(n_{1}\right) \cdots \tau_{\alpha_{k}, \beta_{k}}\left(n_{k}\right)}{\left(n_{1} \cdots n_{k}\right)^{\frac{1}{2}}} V_{\boldsymbol{\alpha}, \boldsymbol{\beta}}\left(\frac{n_{1} \cdots n_{k}}{q^{k}}\right)
\end{aligned}
$$

and the sum over $\epsilon$ is a sum over $\epsilon=\left\{ \pm_{1} 1, \ldots, \pm_{k} 1\right\} \in\{1,-1\}^{k}$.

The diagonal term $\mathcal{D}_{\boldsymbol{\alpha}, \boldsymbol{\beta}}$ will be treated in Section [6, using the results of [Bet]. The terms with $d=1$ in $\mathcal{O}_{\epsilon, \boldsymbol{\alpha}, \boldsymbol{\beta}}^{\prime}$ could be easily dealt with in a simple way, however it is more convenient to keep them together with the other off-diagonal terms.

Lemma 9. We have

$$
\mathcal{D}_{\boldsymbol{\alpha}, \boldsymbol{\beta}}=\mathscr{D}_{\boldsymbol{\alpha}, \boldsymbol{\beta}}+O\left(q^{\frac{k}{2}-\frac{2 k}{k+1}+\varepsilon}\right),
$$

where

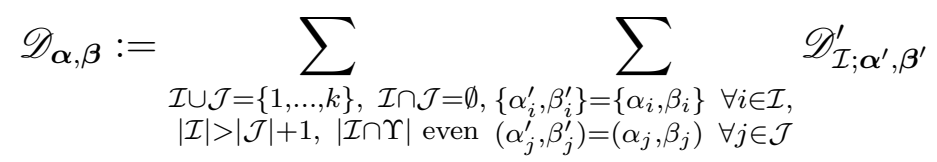

and $\mathscr{D}_{\mathcal{I} ; \boldsymbol{\alpha}, \boldsymbol{\beta}}^{\prime}$ is as defined in (6.2).

For the off-diagonal terms we introduce partitions of unity. We need a function $P: \mathbb{R}_{\geq 0} \rightarrow$ $\mathbb{R}_{\geq 0}$, satisfying

$$
\sum_{N}^{\dagger} P(x / N)=1, \quad \forall x>0
$$


where by $\sum^{\dagger}$ we mean that the index runs through the elements of a certain (fixed) set of positive real numbers such that $\sum_{X^{-1} \leq N \leq X}^{\dagger} 1 \ll \log X$. Also, we require that $P(x)$ is supported on $1 \leq x \leq 2$ and $P^{(j)}(x) \ll j^{A \bar{j}}$ for some $A>0$. It is not difficult to construct such a partition 1 Notice that under these conditions, the Mellin transform of $P(x)$,

$$
\tilde{P}(s):=\int_{0}^{\infty} P(x) x^{s-1} d x
$$

is entire and satisfies

$$
\tilde{P}(\sigma+i t) \ll(1+j+|\sigma|)^{A j} A^{|\sigma|}(1+|t|)^{-j} \quad \forall j \geq 0 .
$$

Using partitions of unity we can decompose $\mathcal{O}_{\boldsymbol{\alpha}, \boldsymbol{\beta}}^{\prime}$ into

$$
\mathcal{O}_{\epsilon, \boldsymbol{\alpha}, \boldsymbol{\beta}}:=\sum_{N_{1}, \cdots, N_{k}}^{\dagger} \mathcal{O}_{\epsilon, \boldsymbol{\alpha}, \boldsymbol{\beta}}^{\prime \prime}\left(N_{1}, \cdots, N_{k}\right)
$$

where $\mathcal{O}_{\epsilon, \boldsymbol{\alpha}, \boldsymbol{\beta}}^{\prime \prime}\left(N_{1}, \cdots, N_{k}\right)$ is defined as $\mathcal{O}_{\epsilon, \boldsymbol{\alpha}, \boldsymbol{\beta}}^{\prime}$, with the only difference that the summands are multiplied by $P\left(n_{1} / N_{1}\right) \cdots P\left(n_{k} / N_{k}\right)$. In the following we will often omit to indicate the dependencies from $N_{1}, \ldots N_{k}$ for ease of notation.

The following two Lemmas summarize our results on the off-diagonal terms. The first Lemma, which is effective when $N_{1}, \cdots, N_{k}$ are close together, uses the spectral theory of automorphic forms (via the bounds proven in Section 3.3) and is proven in Section 7 . The second lemma, which is effective when one of the $N_{i}$ is quite larger than the others, uses the bounds for sums of Kloosterman sums proven by Young in You11b and is proven in Section 9 .

Lemma 10. Let $N_{\max }$ be the maximum among $N_{1}, \ldots, N_{k}$. Then

$$
\sum_{\epsilon \in\{ \pm 1\}^{k}} \rho_{\Upsilon}(\epsilon) \mathcal{O}_{\epsilon, \boldsymbol{\alpha}, \boldsymbol{\beta}}^{\prime \prime}\left(N_{1}, \cdots, N_{k}\right)=\mathcal{M}_{\boldsymbol{\alpha}, \boldsymbol{\beta}}\left(N_{1}, \cdots, N_{k}\right)+\mathcal{E}_{1 ; \boldsymbol{\alpha}, \boldsymbol{\beta}}\left(N_{1}, \cdots, N_{k}\right)
$$

where

$$
\mathcal{E}_{1 ; \boldsymbol{\alpha}, \boldsymbol{\beta}} \ll \frac{N_{\max }^{\varepsilon}}{q^{1-\varepsilon}}\left(\frac{q^{\vartheta} N_{\max }^{\frac{k}{2}+\frac{1}{2}}}{\left(N_{1} \cdots N_{k}\right)^{\frac{1}{2}}}+\frac{q^{\frac{k}{2}-\frac{1}{3}+\frac{\vartheta}{3}} N_{\max }^{\frac{1}{2}}}{\left(N_{1} \cdots N_{k}\right)^{\frac{1}{2}}}+\frac{q^{\frac{1}{6}+\frac{\vartheta}{3}}\left(N_{1} \cdots N_{k}\right)^{\frac{1}{2}}}{N_{\max }}+\frac{\left(N_{1} \cdots N_{k}\right)^{\frac{1}{2}}}{N_{\max }^{\frac{1}{2}}}\right)
$$

and $\mathcal{M}_{\boldsymbol{\alpha}, \boldsymbol{\beta}}\left(N_{1}, \cdots, N_{k}\right)$ is defined in (7.38). Moreover,

$$
\mathcal{M}_{\boldsymbol{\alpha}, \boldsymbol{\beta}}\left(N_{1}, \cdots, N_{k}\right) \ll q^{\varepsilon}\left(N_{1} \cdots N_{k}\right)^{\frac{1}{2}} N_{\max }^{-1+\varepsilon} .
$$

\footnotetext{
${ }^{1}$ For example take the set of indexes in $\sum^{\dagger}$ to be $\left\{\left(\frac{3}{2}\right)^{n} \mid n \in \mathbb{Z}\right\}$ and $P(x)=\int_{1}^{\frac{3}{2}} \eta(x y) \frac{d y}{y}$, where $\eta(x)=$ $C e^{-\frac{1}{1-(4 x-7)^{2}}}$ for $\left|x-\frac{7}{4}\right|<\frac{1}{4}$ and $\eta(x)=0$ otherwise, and where $C$ is such that $\int_{\mathbb{R}} \eta(y) \frac{d y}{y}=1$.
} 
Lemma 11. Let $N_{\max }$ be the maximum among $N_{1}, \ldots, N_{k}$. Then

$$
\mathcal{O}_{\epsilon, \alpha, \beta}^{\prime \prime}\left(N_{1}, \cdots, N_{k}\right) \ll q^{\varepsilon}\left(\left(\frac{N_{1} \cdots N_{k}}{N_{\max }}\right)^{\frac{1}{2}-\frac{1}{2(k-1)}}\left(\frac{q^{\frac{3}{4}}}{N_{\max }^{1 / 2}}+1\right)+\frac{\left(N_{1} \cdots N_{k}\right)^{\frac{1}{2}}}{N_{\max }^{3 / 4}}\right) .
$$

Notice that in the crucial case $N_{1} \cdots N_{k} \asymp q^{k}$ Lemma 10] is non-trivial for $N_{\max } \ll$ $q^{2-2 \frac{\vartheta+1}{k+1}-\delta}$ for any fixed $\delta>0$, whereas Lemma 11] is non-trivial as long as $N_{\max } \gg_{k} q^{\frac{4}{3}+\delta}$. In particular, in order to have a non-trivial bound for all ranges we need $\vartheta<\frac{k-2}{3}$ and so for $k=3$ we need $\theta<\frac{1}{3}$.

The following lemma, which we shall prove in Section 8, allows us to combine the various main terms.

Lemma 12. We have

$$
\begin{aligned}
& \frac{i^{|\Upsilon|}}{2^{k}} \sum_{\substack{N_{1}, \ldots, N_{k}, N_{1} \cdots N_{k} \ll q^{k+\varepsilon}}}^{\dagger}\left(\mathcal{M}_{\boldsymbol{\alpha}, \boldsymbol{\beta}}\left(N_{1}, \cdots, N_{k}\right)+X_{\boldsymbol{\beta}, \boldsymbol{\alpha}} \mathcal{M}_{-\boldsymbol{\beta},-\boldsymbol{\alpha}}\left(N_{1}, \cdots, N_{k}\right)\right) \\
& =-\left(\mathscr{D}_{\boldsymbol{\alpha}, \boldsymbol{\beta}}+X_{\boldsymbol{\beta}, \boldsymbol{\alpha}} \mathscr{D}_{-\boldsymbol{\beta},-\boldsymbol{\alpha}}\right)+\sum_{\left\{\alpha_{i}^{\prime}, \beta_{i}^{\prime}\right\}=\left\{\alpha_{i}, \beta_{i}\right\}} \mathscr{M}_{\boldsymbol{\alpha}^{\prime}, \boldsymbol{\beta}^{\prime}}+O\left(q^{\frac{k}{2}-\frac{3}{2}+\iota_{k}+\varepsilon}\right),
\end{aligned}
$$

where $\mathcal{M}_{\alpha, \beta}$ is as defined in (2.3) and $\iota_{k}=\frac{3}{14}$ if $k=4$ and $\iota_{k}=0$ otherwise.

We conclude the section with the deduction of Theorem 4 from the above lemmas.

Proof of Theorem 4. First we remark that by (5.4), we can rewrite Lemma 11 as

$$
\sum_{\epsilon \in\{ \pm 1\}^{k}} \rho_{\Upsilon}(\epsilon) \mathcal{O}_{\epsilon, \alpha, \beta}^{\prime \prime}\left(N_{1}, \cdots, N_{k}\right)=\mathcal{M}_{\alpha, \beta}\left(N_{1}, \cdots, N_{k}\right)+\mathcal{E}_{2 ; \alpha, \beta}\left(N_{1}, \cdots, N_{k}\right)
$$

where

$$
\mathcal{E}_{2 ; \alpha, \beta} \ll q^{\varepsilon}\left(\left(\frac{N_{1} \cdots N_{k}}{N_{\max }}\right)^{\frac{1}{2}-\frac{1}{2(k-1)}}\left(\frac{q^{\frac{3}{4}}}{N_{\max }^{1 / 2}}+1\right)+\frac{\left(N_{1} \cdots N_{k}\right)^{\frac{1}{2}}}{N_{\max }^{3 / 4}}\right) .
$$

Moreover, we also have

$$
\sum_{\epsilon \in\{ \pm 1\}^{k}} \rho_{\Upsilon}(\epsilon) \mathcal{O}_{\epsilon, \alpha, \beta}^{\prime \prime}\left(N_{1}, \cdots, N_{k}\right)=\mathcal{M}_{\alpha, \beta}\left(N_{1}, \cdots, N_{k}\right)+\mathcal{E}_{3 ; \alpha, \beta}\left(N_{1}, \cdots, N_{k}\right),
$$

with $\mathcal{E}_{3 ; \alpha, \beta}\left(N_{1}, \cdots, N_{k}\right) \ll q^{-1+\varepsilon}\left(N_{1} \cdots N_{k}\right)^{\frac{1}{2}}$, since both $O_{\epsilon, \alpha, \beta}^{\prime \prime}$ and $\mathcal{M}_{\alpha, \beta}$ satisfy trivially such a bound. Thus, by Lemma 12 (and adding the condition $N_{1} \cdots N_{k} \ll q^{k+\varepsilon}$ at a negligible 
cost) we have

$$
\begin{aligned}
\mathcal{O}_{\boldsymbol{\alpha}, \boldsymbol{\beta}} & =\frac{i^{|\Upsilon|}}{2^{k}} \sum_{\epsilon \in\{ \pm 1\}^{k}} \rho_{\Upsilon}(\epsilon) \sum_{\substack{N_{1}, \ldots, N_{k}, N_{1} \cdots N_{k} \ll q^{k+\varepsilon}}}^{\dagger}\left(\mathcal{O}_{\alpha, \beta}\left(N_{1}, \cdots, N_{k}\right)+X_{\boldsymbol{\beta}, \boldsymbol{\alpha}} \mathcal{O}_{-\boldsymbol{\beta},-\boldsymbol{\alpha}}\left(N_{1}, \cdots, N_{k}\right)\right)+O(1) \\
& =-\left(\mathscr{D}_{\boldsymbol{\alpha}, \boldsymbol{\beta}}+X_{\boldsymbol{\beta}, \boldsymbol{\alpha}} \mathscr{D}_{-\boldsymbol{\beta},-\boldsymbol{\alpha}}\right)+\sum_{\left\{\alpha_{i}^{\prime}, \beta_{i}^{\prime}\right\}=\left\{\alpha_{i}, \beta_{i}\right\}} \mathcal{M}_{\boldsymbol{\alpha}^{\prime}, \boldsymbol{\beta}^{\prime}}+\mathscr{E}_{\boldsymbol{\alpha}, \boldsymbol{\beta}}+O\left(q^{\frac{k}{2}-\frac{3}{2}+\iota_{k}+\varepsilon}\right),
\end{aligned}
$$

where

$$
\mathscr{E}_{\boldsymbol{\alpha}, \boldsymbol{\beta}} \ll \max _{\substack{N_{1}, \ldots, N_{k} \\ N_{1} \cdots N_{k} \ll q^{k+\varepsilon}}}\left(\min \left(\mathscr{E}_{1}, \mathscr{E}_{2}, \mathscr{E}_{3}\right)\right)
$$

Thus, since the term $-\left(\mathscr{D}_{\boldsymbol{\alpha}, \boldsymbol{\beta}}+X_{\boldsymbol{\beta}, \boldsymbol{\alpha}} \mathscr{D}_{-\boldsymbol{\beta},-\boldsymbol{\alpha}}\right)$ cancels out with the main term of the diagonal term given by (5.1) , to conclude the proof of Theorem 4 we just need to show that $\mathscr{E}_{\boldsymbol{\alpha}, \boldsymbol{\beta}} \ll$ $q^{\frac{k}{2}-1-\delta_{k}+\varepsilon}$. Writing $N_{\max }=q^{a}$ and $N_{1} \cdots N_{k}=q^{b}$ (and considering only the contribution from the first summand in (5.3), since it easy to see the other terms produce a contribution which is $O\left(q^{\frac{k}{2}-\frac{3}{2}+\varepsilon}\right)$ ), we have that it is sufficient to show that

$$
\max _{i=1,2,3} \max _{\substack{a \leq b \leq k, k a \geq b}} \min \left(\frac{k+1}{2} a-\frac{b}{2}-1+\vartheta, L_{i}(a, b), \frac{b}{2}-1\right)=\frac{k}{2}-\frac{3}{2}+\frac{3(3+2 \vartheta)}{2(2 k+5)}=\frac{k}{2}-1-\delta_{k},
$$

for $k \geq 3$, where

$L_{1}(a, b):=\frac{3}{4}-\frac{a}{2}+(b-a)\left(\frac{1}{2}-\frac{1}{2(k-1)}\right), \quad L_{2}(a, b):=(b-a)\left(\frac{1}{2}-\frac{1}{2(k-1)}\right), \quad L_{3}(a, b):=\frac{b}{2}-\frac{3}{4} a$.

If the maximum is attained at the interior of $\{a \leq b \leq k, k a \geq b\}$, then it must occur when $\frac{k+1}{2} a-\frac{b}{2}-1=\frac{b}{2}-1=L_{i}(a, b)$ for $i=1,2$, or 3 and so it would be $\frac{7 k}{20}-\frac{13}{20}, \frac{k}{3}-\frac{2}{3}$ and $\frac{k}{3}-\frac{2}{3}$ respectively. Along the lines $a=b, k a=b$ and $b=k$ we have

$$
\begin{aligned}
& \max _{i=1,2,3} \max _{0 \leq a \leq k} \min \left(\frac{k}{2} a-1+\vartheta, L_{i}(a, a), \frac{a}{2}-1\right)=\max _{0 \leq a \leq k} \min \left(L_{i}(a, a), \frac{a}{2}-1\right)=0, \\
& \max _{i=1,2,3} \max _{0 \leq a \leq 1} \min \left(\frac{a}{2}-1+\vartheta, L_{i}(a, k a), \frac{k a}{2}-1\right) \leq-\frac{1}{2}+\vartheta \leq 0 \\
& \max _{i=1,2,3} \max _{1 \leq a \leq k} \min \left(\frac{k+1}{2} a-\frac{k}{2}-1+\vartheta, L_{i}(a, k), \frac{k}{2}-1\right) \\
& \quad=\max \left(\frac{k}{2}-\frac{7}{4}+\frac{8 k(2+\vartheta)-19-12 \vartheta}{4\left(k^{2}+2 k-4\right)}, \frac{k}{2}-\frac{3}{2}+\frac{2(k+\vartheta)-5-4 \vartheta}{2\left(k^{2}+k-3\right)}, \frac{k}{2}-\frac{3}{2}+\frac{3(3+2 \vartheta)}{2(2 k+5)}\right)=\frac{k}{2}-\frac{3}{2}+\frac{3(3+2 \vartheta)}{2(2 k+5)},
\end{aligned}
$$

for $k \geq 3$ and $\vartheta \leq \frac{1}{3}$. Theorem 4 then follows. 


\section{The diagonal terms}

In this section we prove Lemma 9 deducing it from the following Lemma in Bet. We recall that in Section 4 we assumed $\left|\alpha_{i}\right|,\left|\beta_{i}\right|<\frac{\varepsilon}{2 k}$ for all $i=1, \ldots, k$.

Lemma 13. For $\Re(s)>1-\frac{1}{k}-\frac{1}{k} \sum_{i=1}^{k} \min \left(\Re\left(\alpha_{i}\right), \Re\left(\beta_{i}\right)\right)$, let

$$
\mathcal{W}_{\alpha, \beta}(s):=\frac{i^{|\Upsilon|}}{2^{k}} \sum_{\epsilon \in\{ \pm 1\}^{k}} \rho_{\Upsilon}(\epsilon) \sum_{ \pm_{1} n_{1}+\cdots+ \pm_{k} n_{k}=0} \frac{\tau_{\alpha_{1}, \beta_{1}}\left(n_{1}\right) \cdots \tau_{\alpha_{k}, \beta_{k}}\left(n_{k}\right)}{\left(n_{1} \cdots n_{k}\right)^{s}} .
$$

Also, let

$$
\begin{aligned}
\mathcal{W}_{\alpha, \beta}^{\dagger}(s):= & \sum_{\left(\mathcal{I}, \boldsymbol{\alpha}^{\prime}, \boldsymbol{\beta}^{\prime}\right) \in S_{\boldsymbol{\alpha}, \boldsymbol{\beta}}} \frac{2^{|\mathcal{J}|+1} \pi^{\frac{|\mathcal{I}|}{2}-1}}{|\mathcal{I}|(s-1)+s_{\mathcal{I} ; \boldsymbol{\alpha}^{\prime}}+1}\left(\prod_{i \in \mathcal{I}} \zeta\left(1-\alpha_{i}^{\prime}+\beta_{i}^{\prime}\right) \frac{\Gamma_{i}\left(-\frac{\alpha_{i}^{\prime}}{2}+\frac{1+s_{\mathcal{I} ; \boldsymbol{\alpha}^{\prime}}}{2|\mathcal{I}|}\right)}{\Gamma_{i}\left(\frac{1}{2}+\frac{\alpha_{i}^{\prime}}{2}-\frac{1+s_{\mathcal{I} ; \boldsymbol{\alpha}^{\prime}}}{2|\mathcal{I}|}\right)}\right) \times \\
& \times \sum_{\ell \geq 1} \sum_{h(\bmod \ell)}^{*} \frac{1}{\ell^{\left|\mathcal{I}^{\prime}\right|-\sum_{i \in \mathcal{I}}\left(\alpha_{i}^{\prime}-\beta_{i}^{\prime}\right)}} \prod_{i \notin \mathcal{I}} D_{i}\left(1+\alpha_{i}^{\prime}-\frac{1+s_{\mathcal{I} ; \boldsymbol{\alpha}^{\prime}}}{|\mathcal{I}|}, \alpha_{i}^{\prime}-\beta_{i}^{\prime}, \frac{h}{\ell}\right) .
\end{aligned}
$$

where $s_{\mathcal{I} ; \boldsymbol{\alpha}^{\prime}}:=\sum_{i \in \mathcal{I}} \alpha_{i}^{\prime}$ and

$$
S_{\boldsymbol{\alpha}, \boldsymbol{\beta}}:=\left\{\left(\mathcal{I}, \boldsymbol{\alpha}^{\prime}, \boldsymbol{\beta}^{\prime}\right) \mid \begin{array}{l}
\mathcal{I} \subseteq\{1, \ldots k\},|\mathcal{I}|>|\mathcal{J}|+1,|\mathcal{I} \cap \Upsilon| \text { even, } \\
\left\{\alpha_{i}^{\prime}, \beta_{i}^{\prime}\right\}=\left\{\alpha_{i}, \beta_{i}\right\} \forall i \in \mathcal{I}, \quad\left(\alpha_{i}^{\prime}, \beta_{i}^{\prime}\right)=\left(\alpha_{i}, \beta_{i}\right) \forall i \notin \mathcal{I}
\end{array}\right\} .
$$

Then for any $\varepsilon>0 \mathcal{W}_{\alpha, \beta}(s)-\mathcal{W}_{\alpha, \beta}^{\dagger}(s)$ extends to an holomorphic function on $\Re(s) \geq 1-\frac{2-4 \varepsilon}{k+1}$ and in such half plane it satisfies $\mathcal{W}_{\alpha, \beta}(s)-\mathcal{W}_{\alpha, \beta}^{\dagger}(s) \ll\left(\frac{k}{\varepsilon}(1+|s|)\right)^{A k}$.

Proof. Theorem 3 of [Bet] gives the meromorphic continuation and the bound for each $\epsilon$. Thus, one obtains the Lemma by summing over $\epsilon$ (for the simplification of the polar term one proceeds as in Lemma [19; cf. also Remark 2 of [Bet]).

Proof of Lemma 9. Writing $V_{\boldsymbol{\alpha}, \boldsymbol{\beta}}$ in terms of it's Mellin transform we have

$$
\mathcal{D}_{\boldsymbol{\alpha}, \boldsymbol{\beta}}=\frac{1}{2 \pi i} \int_{(2)} G_{\boldsymbol{\alpha}, \boldsymbol{\beta}}(s) g_{\boldsymbol{\alpha}, \boldsymbol{\beta}}(s) \mathcal{W}_{\alpha, \beta}\left(\frac{1}{2}+s\right) q^{k s} \frac{d s}{s} .
$$

We write $\mathcal{W}_{\alpha, \beta}\left(\frac{1}{2}+s\right)$ as $\mathcal{W}_{\alpha, \beta}^{\dagger}\left(\frac{1}{2}+s\right)+\left(\mathcal{W}_{\alpha, \beta}\left(\frac{1}{2}+s\right)-\mathcal{W}_{\alpha, \beta}^{\dagger}\left(\frac{1}{2}+s\right)\right)$. For the second term we move the line of integration to $\Re(s)=\frac{1}{2}-\frac{2-4 \varepsilon}{k+1}$ and bound trivially using (4.2) obtaining an error of size $O\left(k^{A k} q^{\frac{k}{2}-\frac{2 k}{k+1}+\varepsilon}\right)=O\left(q^{\frac{k}{2}-\frac{2 k}{k+1}+\varepsilon}\right)$. For the first term we move the line of integration to $\Re(s)=-\frac{1}{2}$ picking up the residues from the poles. We obtain

$$
\mathcal{D}_{\boldsymbol{\alpha}, \boldsymbol{\beta}}=\sum_{\substack{\mathcal{I} \cup \mathcal{J}=\{1, \ldots, k\}, \mathcal{I} \cap \mathcal{J}=\emptyset,\left\{\alpha_{i}^{\prime}, \beta_{i}^{\prime}\right\}=\left\{\alpha_{i}, \beta_{i}\right\} \\|\mathcal{I}|>|\mathcal{J}|+1,|\mathcal{I} \cap \Upsilon| \text { even }\left(\alpha_{j}^{\prime}, \beta_{j}^{\prime}\right)=\left(\alpha_{j}, \beta_{j}\right) \forall j \in \mathcal{J},}} \mathscr{D}_{\mathcal{I} ; \boldsymbol{\alpha}^{\prime}, \boldsymbol{\beta}^{\prime}}+O\left(q^{\frac{k}{2}-\frac{2 k}{k+1}+\varepsilon}\right),
$$


where

$$
\begin{aligned}
& \mathscr{D}_{\mathcal{I} ; \boldsymbol{\alpha}, \boldsymbol{\beta}}=2^{k} \frac{G_{\boldsymbol{\alpha}, \boldsymbol{\beta}}\left(s_{\mathcal{I} ; \boldsymbol{\alpha}}\right)}{s_{\mathcal{I} ; \boldsymbol{\alpha}} \pi|\mathcal{I}|} g_{\boldsymbol{\alpha}, \boldsymbol{\beta}}\left(s_{\mathcal{I} ; \boldsymbol{\alpha}}\right) q^{k s_{\mathcal{I} ; \boldsymbol{\alpha}}}\left(\prod_{i \in \mathcal{I}} \frac{\pi^{\frac{1}{2}} \zeta\left(1-\alpha_{i}+\beta_{i}\right)}{2^{\frac{1}{2}+\alpha_{i}+s_{\mathcal{I}, \boldsymbol{\alpha}}}} \frac{\Gamma_{i}\left(\frac{1}{4}-\frac{\alpha_{i}+s_{\mathcal{I}, \boldsymbol{\alpha}}}{2}\right)}{\Gamma_{i}\left(\frac{1}{4}+\frac{\alpha_{i}+s_{\mathcal{I}, \boldsymbol{\alpha}}}{2}\right)}\right) \times \\
& \times \sum_{\ell} \sum_{h(\bmod \ell)}^{*} \frac{1}{\ell^{|\mathcal{I}|-\sum_{i \in \mathcal{I}}\left(\alpha_{i}-\beta_{i}\right)}}\left(\prod_{j \in \mathcal{J}} D_{j}\left(\frac{1}{2}+\alpha_{j}+s_{\mathcal{I}, \boldsymbol{\alpha}}, \alpha_{j}-\beta_{j}, \pm_{j} \frac{h}{\ell}\right)\right)
\end{aligned}
$$

with $s_{\mathcal{I} ; \boldsymbol{\alpha}}:=\sum_{i \in \mathcal{I}} \alpha_{i}$.

\section{The terms close to the diagonal}

In this section we prove Lemma 10. First, we assume that $N_{1}$ is the maximum of $N_{1}, \ldots, N_{k}$, as we can do since both the main term and the error terms in Lemma 10 are symmetric in the indexes. Moreover, since we assumed that $|\Upsilon|$ is even, then we have

$$
\sum_{\epsilon \in\{ \pm 1\}^{k}} \rho_{\Upsilon}(\epsilon) \mathcal{O}_{\epsilon, \alpha, \beta}^{\prime \prime}=2 \sum_{\substack{\epsilon \in\{ \pm 1\}^{k} \\ \pm 11=-1}} \rho_{\Upsilon}(\epsilon) \mathcal{O}_{\epsilon, \boldsymbol{\alpha}, \boldsymbol{\beta}}^{\prime \prime},
$$

where here and in the following $\epsilon=\left( \pm_{1} 1, \pm_{2} 1, \ldots, \pm_{k} 1\right)$. We split $\mathcal{O}_{\epsilon, \alpha, \beta}^{\prime \prime}$ further, depending on the sign and the size of $\pm_{*} f:=-n_{1} \pm_{2} n_{2} \pm_{3} \cdots \pm_{k} n_{k}$ (with $f>0$ ), introducing another partition of unity controlling the size of $f$ :

$$
\sum_{\epsilon \in\{ \pm 1\}^{k}} \rho_{\Upsilon}(\epsilon) \mathcal{O}_{\epsilon, \boldsymbol{\alpha}, \boldsymbol{\beta}}^{\prime \prime}=2 \sum_{N_{*} \ll k N_{1} q^{\varepsilon / k}}^{\dagger} \sum_{\substack{\epsilon \in\{ \pm 1\}^{k} \\ \pm_{1}=-1}} \rho_{\Upsilon}(\epsilon) \sum_{ \pm_{*} 1 \in\{ \pm 1\}} \mathcal{K}_{\epsilon, \pm_{*}, \boldsymbol{\alpha}, \boldsymbol{\beta}}
$$

where

$$
\begin{aligned}
\mathcal{K}_{\epsilon, \pm_{*} ; \boldsymbol{\alpha}, \boldsymbol{\beta}}:= & \sum_{d \mid q} d \frac{\mu\left(\frac{q}{d}\right)}{\varphi(q)} \sum_{\substack{f \geq 1, f \equiv 0(\bmod d)}} \sum_{\substack{n_{1}, \ldots, n_{k} \geq 1, n_{1}= \pm_{2} n_{2} \pm_{3} \cdots \pm_{k} n_{k} \pm_{*} f}} \frac{\tau_{\alpha_{1}, \beta_{1}}\left(n_{1}\right) \cdots \tau_{\alpha_{k}, \beta_{k}}\left(n_{k}\right)}{\left(n_{1} \cdots n_{k}\right)^{\frac{1}{2}}} \times \\
& \times V_{\boldsymbol{\alpha}, \boldsymbol{\beta}}\left(\frac{n_{1} \cdots n_{k}}{q^{k}}\right) P\left(\frac{n_{1}}{N_{1}}\right) \cdots P\left(\frac{n_{k}}{N_{k}}\right) P\left(\frac{f}{N_{*}}\right) .
\end{aligned}
$$

Notice that in (7.1) we truncated the sum over $N_{*}$ at $N_{*} \ll k N_{1} q^{\varepsilon / k}$, as we clearly could.

\subsection{Separating the variables arithmetically}

We wish to separate the variables in $\tau_{\alpha_{1}, \beta_{1}}\left(n_{1}\right)=\tau_{\alpha_{1}, \beta_{1}}\left( \pm_{2} n_{2} \pm_{3} \cdots \pm_{k} n_{k} \pm_{*} f\right)$. One can achieve this goal by using Ramanujan's identity

$$
\tau_{a, b}(n)=n^{-a} \tau_{0, b-a}(n)=n^{-a} \zeta(1-a+b) \sum_{\ell=1}^{\infty} \frac{c_{\ell}(n)}{\ell^{1-a+b}},
$$


which holds for $n \neq 0$ and $\Re(a-b)<0$. The coefficient $c_{\ell}(n)$ denotes the Ramanujan sum

$$
c_{\ell}(n):=\sum_{h(\bmod \ell)}^{*} \mathrm{e}\left(\frac{n h}{\ell}\right)
$$

However, since (7.2) doesn't hold in a neighborhood of $a=b=0$, it is more convenient to follow Young's approach and use the following Lemma, which rephrase (7.2) as an approximate functional equation for $\tau_{a, b}(n)$.

Lemma 14. Let $n \in \mathbb{Z}_{>0}$ and let $a, b \in \mathbb{C}$. Then,

$$
\tau_{a, b}(n)=n^{-a} \sum_{\ell} \frac{c_{\ell}(n)}{\ell^{1-a+b}} v_{a-b}\left(\frac{\ell^{2}}{n}\right)+n^{-b} \sum_{\ell} \frac{c_{\ell}(n)}{\ell^{1+a-b}} v_{b-a}\left(\frac{\ell^{2}}{n}\right)
$$

where

$$
v_{a}(x)=\int_{\left(c_{w}\right)} x^{-\frac{w}{2}} \zeta(1-a+w) \frac{G_{\boldsymbol{\alpha}, \boldsymbol{\beta}}(w)}{w} d w
$$

where $c_{w}>|\Re(a-b)|$ and $G_{\boldsymbol{\alpha}, \boldsymbol{\beta}}(w)$ is as defined in (4.1).

Proof. See Lemma 5.4 of Young [You11b].

Applying (17.3) and splitting the resulting sum over $\ell$ using another partition of unity (and adding the restriction $L \geq \frac{1}{2}$ as we can do since $P$ is supported on $[1,2]$ ), we rewrite $\mathcal{K}_{\epsilon, \pm_{*} ; \boldsymbol{\alpha}, \boldsymbol{\beta}}$ as

$$
\mathcal{K}_{\epsilon, \pm * ; \boldsymbol{\alpha}, \boldsymbol{\beta}}=\sum_{\substack{\left\{\alpha_{1}^{\prime}, \beta_{1}^{\prime}\right\}=\left\{\alpha_{1}, \beta_{1}\right\},\left(\alpha_{j}^{\prime}, \beta_{j}^{\prime}\right)=\left(\alpha_{j}, \beta_{j}\right) \forall j \neq 1}} \sum_{L \geq \frac{1}{2}}^{\dagger} \mathcal{L}_{\boldsymbol{\alpha}^{\prime}, \boldsymbol{\beta}^{\prime}}
$$

where $\boldsymbol{\alpha}^{\prime}:=\left(\alpha_{1}^{\prime}, \ldots, \alpha_{k}^{\prime}\right), \boldsymbol{\beta}^{\prime}:=\left(\beta_{1}^{\prime}, \ldots, \beta_{k}^{\prime}\right)$ and

$$
\begin{aligned}
\mathcal{L}_{\boldsymbol{\alpha}, \boldsymbol{\beta}}:= & \sum_{\substack{n_{1}, \ldots, n_{k}, f \geq 1, n_{1}= \pm_{2} n_{2} \pm_{3} \cdots \pm_{k} n_{k} \pm_{*} f}} \sum_{\ell} \sum_{h(\bmod \ell)}^{*} \frac{c_{\ell}\left( \pm_{2} n_{2} \pm_{3} \cdots \pm_{k} n_{k} \pm_{*} f\right)}{\ell^{1-\alpha+\beta}} v_{\alpha_{1}-\beta_{1}}\left(\frac{\ell^{2}}{n_{1}}\right) \times \\
& \times \frac{\sigma_{\alpha_{2}-\beta_{2}}\left(n_{2}\right) \cdots \sigma_{\alpha_{k}-\beta_{k}}\left(n_{k}\right)}{n_{1}^{\frac{1}{2}+\alpha_{1}+s} \cdots n_{k}^{\frac{1}{2}+\alpha_{k}+s}} V_{\boldsymbol{\alpha}, \boldsymbol{\beta}}\left(\frac{n_{1} \cdots n_{k}}{q^{k}}\right) P\left(\frac{n_{1}}{N_{1}}\right) \cdots P\left(\frac{n_{k}}{N_{k}}\right) P\left(\frac{f}{N_{*}}\right) P\left(\frac{\ell}{L}\right) .
\end{aligned}
$$

Notice that we have omitted to indicate the dependency of $\mathcal{L}_{\boldsymbol{\alpha}, \boldsymbol{\beta}}$ from $\epsilon$ and $\pm_{*}$ in order to save notation. 
Expressing $P, v_{\alpha_{1}-\beta_{1}}$ and $V$ in terms of their Mellin transform and making the change of variables $u_{i} \rightarrow u_{i}-s$, for $i=1, \ldots, k$, we see that $\mathcal{L}_{\boldsymbol{\alpha}, \boldsymbol{\beta}}$ can be written as

$$
\begin{aligned}
& \mathcal{L}_{\boldsymbol{\alpha}, \boldsymbol{\beta}}=\sum_{d \mid q} \frac{\mu\left(\frac{q}{d}\right) d}{\varphi(q)} \sum_{\substack{n_{1}, \ldots, n_{k}, f \geq 1, d \mid f, \pm_{2} n_{2} \pm_{3} \cdots \pm_{k} n_{k} \pm_{*} f>0}} \sum_{\ell} \sum_{h(\bmod \ell)}^{*} \frac{1}{(2 \pi i)^{k+3}} \int_{\substack{\left(\begin{array}{c}
c_{s}, c_{w}, \boldsymbol{c}_{\boldsymbol{u}}, c_{u_{*}}
\end{array} \\
f^{u_{2}}\right.}} \frac{N_{*}^{u_{*}}}{f^{u_{*}}} P\left(\frac{\ell}{L}\right) \\
& \times \frac{N_{1}^{u_{1}-s} \cdots N_{k}^{u_{k}-s}}{\ell^{1-\alpha_{1}+\beta_{1}+w}} \frac{\tau_{\alpha_{2}, \beta_{2}}\left(n_{2}\right) \cdots \tau_{\alpha_{k}, \beta_{k}}\left(n_{k}\right) c_{\ell}\left( \pm_{2} n_{2} \pm_{3} \cdots \pm_{k} n_{k} \pm_{*} f\right)}{\left( \pm_{2} n_{2} \pm_{3} \cdots \pm_{k} n_{k} \pm_{*} f\right)^{\frac{1}{2}+\alpha_{1}+u_{1}-\frac{w}{2}} n_{2}^{\frac{1}{2}+u_{2}} \cdots n_{k}^{\frac{1}{2}+u_{k}}} \\
& \times \tilde{P}\left(u_{*}\right) \tilde{P}\left(u_{1}-s\right) \cdots \tilde{P}\left(u_{k}-s\right) q^{k s} \frac{H_{\boldsymbol{\alpha}, \boldsymbol{\beta}}(w, s)}{w s} d w d s \boldsymbol{d u} d u_{*},
\end{aligned}
$$

where $\boldsymbol{d} \boldsymbol{u}:=d u_{1} \cdots d u_{k}, \boldsymbol{c}_{\boldsymbol{u}}$ denotes the lines of integration $c_{u_{1}}, \ldots, c_{u_{k}}$ and

$$
H_{\boldsymbol{\alpha}, \boldsymbol{\beta}}(w, s):=\zeta\left(1+w-\alpha_{1}+\beta_{1}\right) G_{\boldsymbol{\alpha}, \boldsymbol{\beta}}(s) G_{\boldsymbol{\alpha}, \boldsymbol{\beta}}(w) g_{\boldsymbol{\alpha}, \boldsymbol{\beta}}(s)
$$

Notice that, by the definitions (4.1) and (3.7) of $G_{\boldsymbol{\alpha}, \boldsymbol{\beta}}(s)$ and $g_{\boldsymbol{\alpha}, \boldsymbol{\beta}}(s), H_{\boldsymbol{\alpha}, \boldsymbol{\beta}}(w, s)$ is entire and decays rapidly in both variables $w$ and $s$ :

$$
H_{\boldsymbol{\alpha}, \boldsymbol{\beta}}(w, s) \ll e^{-C_{2}(|\Im(s)|+|\Im(w)|}(1+|\Re(s)|+|\Re(w)|)^{A(|\Re(s)|+|\Re(w)|+k)},
$$

for some $C_{2}>0$. As lines of integration, we take

$$
c_{s}:=\varepsilon / k, \quad c_{u_{1}}=-3 k-\frac{1}{2}-\alpha_{1}+7 \varepsilon, \quad c_{u_{*}}=c_{u_{2}}=\cdots=c_{u_{k}}=4 k, \quad c_{w}=10 \varepsilon
$$

\subsection{Separating the variables analytically}

To complete the separation of the variables, we need also to deal with the factor $\left( \pm_{2} n_{2} \pm_{3}\right.$ $\left.\cdots \pm_{k} n_{k} \pm_{*} f\right)^{\frac{1}{2}+\alpha_{1}+u_{1}-\frac{w}{2}}$ in (7.5). In order to do so, we use Lemma 23, in Section 10, We apply the lemma with $\kappa:=k+1, B:=3 k$ and $v_{1}=\frac{1}{2}-\alpha_{1}-u_{1}+\frac{w}{2}$, so that $\Re\left(v_{1}\right)=B+1-2 \varepsilon$. We get

$$
\mathcal{L}_{\boldsymbol{\alpha}, \boldsymbol{\beta}}=\sum_{\nu} \frac{B !}{\nu_{2} ! \cdots \nu_{k} ! \nu_{*} !}\left(\mathcal{N}_{\nu ; \boldsymbol{\alpha}, \boldsymbol{\beta}}+\mathcal{N}_{\nu ; \boldsymbol{\alpha}, \boldsymbol{\beta}}^{\prime}\right)
$$

where the sum is over $\nu=\left(\nu_{2}, \ldots, \nu_{k}, \nu_{*}\right) \in \mathbb{Z}_{\geq 0}^{k}$ satisfying

$$
\nu_{2}+\cdots+\nu_{k}+\nu_{*}=B, \quad \nu_{i}=0 \text { if } \pm_{i}=-1, \quad \nu_{*}=0 \text { if } \pm_{*}=-1
$$


and $\mathcal{N}_{\nu ; \boldsymbol{\alpha}, \boldsymbol{\beta}}$ is defined by

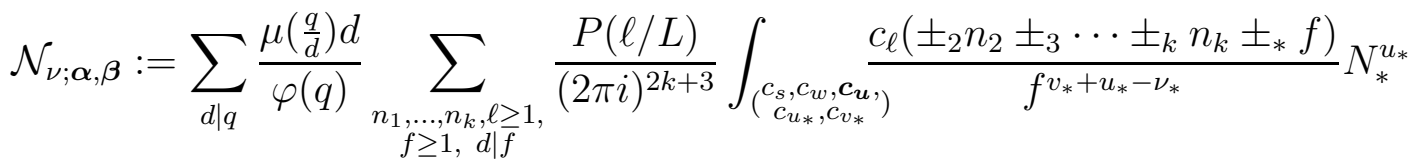

$$
\begin{aligned}
& \times \frac{q^{k s} N_{1}^{u_{1}-s}}{\ell^{1-\alpha_{1}+\beta_{1}+w}} \tilde{P}\left(u_{*}\right) \tilde{P}\left(u_{1}-s\right)\left(\prod_{i=2}^{k} \int_{\left(c_{v_{i}}\right)} \frac{\tau_{\alpha_{i}, \beta_{i}}\left(n_{i}\right) N_{i}^{u_{i}-s}}{n_{i}^{\frac{1}{2}+u_{i}+v_{i}-\nu_{i}}} \tilde{P}\left(u_{i}-s\right)\right) \\
& \times \Psi_{\epsilon^{*}, B}\left(\frac{1}{2}-\alpha_{1}-u_{1}+\frac{w}{2}, \boldsymbol{v}, v_{*}\right) \frac{H_{\boldsymbol{\alpha}, \boldsymbol{\beta}}(w, s)}{w s} d s d w \boldsymbol{d u d} \boldsymbol{v} d u_{*} d v_{*},
\end{aligned}
$$

with $c_{v_{2}}=\cdots=c_{v_{k}}=c_{v_{*}}=\varepsilon / k$, and $\mathcal{N}_{\nu ; \boldsymbol{\alpha}, \boldsymbol{\beta}}^{\prime}$ is defined in the same way with lines of integrations $c_{v_{2}}^{\prime}=\cdots=c_{v_{k}}^{\prime}=c_{v_{*}}^{\prime}=\frac{1}{2}$ in place of $c_{v_{2}}, \ldots, c_{v_{k}}, c_{v_{*}}$. Also, in (7.8) we used the notation $\boldsymbol{v}:=\left(v_{2}, \ldots, v_{k}\right), \boldsymbol{d} \boldsymbol{v}:=d v_{2} \cdots d v_{k}$ and $\epsilon^{*}:=\left( \pm_{1} 1, \ldots, \pm_{k} 1, \pm_{*} 1\right)$.

The contribution of $\mathcal{N}_{\nu ; \boldsymbol{\alpha}, \boldsymbol{\beta}}^{\prime}$ can be bounded by moving the lines of integration $c_{u_{i}}$ to $c_{u_{i}}=2 \varepsilon+\nu_{i}$ for $i=2, \ldots, k$ and $c_{u_{*}}$ to $c_{u_{*}}=\frac{1}{2}+\nu_{*}+\varepsilon$ and bounding trivially. We obtain

$$
\mathcal{N}_{\nu ; \boldsymbol{\alpha}, \boldsymbol{\beta}}^{\prime} \ll q^{-1+\varepsilon} N_{1}^{-B-\frac{1}{2}+A \varepsilon} N_{*}^{\frac{1}{2}+\nu_{*}+2 \varepsilon} N_{2}^{\nu_{2}+2 \varepsilon} \cdots N_{k}^{\nu_{k}+2 \varepsilon} L^{-\varepsilon}
$$

and thus

$$
\sum_{\nu} \frac{B !}{\nu_{2} ! \cdots \nu_{k} ! \nu_{*} !} \mathcal{N}_{\nu ; \boldsymbol{\alpha}, \boldsymbol{\beta}}^{\prime} \ll q^{-1+A \varepsilon} N_{1}^{A \varepsilon} L^{-\varepsilon}
$$

since $N_{1}$ is the maximum among $N_{1}, \ldots, N_{k}$ and $N_{*} \ll k N_{1} q^{\varepsilon / k}$.

Next, we open the Ramanujan sum in (7.8) and we execute the sums over $n_{2}, \ldots, n_{k}, f$ as we can do since the integrals and sums are absolutely convergent. We obtain

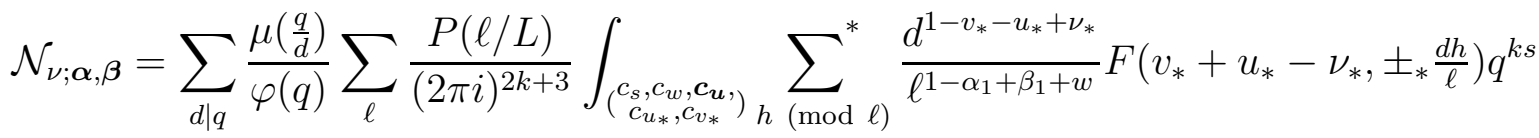

$$
\begin{aligned}
& \times N_{*}^{u_{*}} \tilde{P}\left(u_{*}\right)\left(\prod_{i=2}^{k} \int_{\left(c_{v_{i}}\right)} D_{\alpha_{i}, \beta_{i}}\left(\frac{1}{2}+u_{i}+v_{i}-\nu_{i}, \frac{ \pm_{i} h}{\ell}\right) \tilde{P}\left(u_{i}-s\right) N_{i}^{u_{i}-s}\right) \\
& \times N_{1}^{u_{1}-s} \tilde{P}\left(u_{1}-s\right) \Psi_{\epsilon^{*}, B}\left(\frac{1}{2}-\alpha_{1}-u_{1}+\frac{w}{2}, \boldsymbol{v}, v_{*}\right) \frac{H_{\boldsymbol{\alpha}, \boldsymbol{\beta}}(w, s)}{w s} d s d w \boldsymbol{d u d} \boldsymbol{d} d u_{*} d v_{*},
\end{aligned}
$$

where, after moving the lines of integration $c_{u_{2}}, \ldots, c_{u_{k}}, c_{u_{*}}$, we have

$$
\begin{aligned}
& c_{s}:=\varepsilon / k, \quad c_{u_{1}}=-B-\frac{1}{2}-\Re\left(\alpha_{1}\right)+7 \varepsilon, \quad c_{w}=10 \varepsilon, \\
& c_{v_{2}}=\cdots=c_{v_{k}}=c_{v_{*}}=\varepsilon / k, \quad c_{u_{*}}=1+\nu_{*}+2 \varepsilon-\varepsilon / k
\end{aligned}
$$

and $c_{u_{i}}=\frac{1}{2}+\nu_{i}+\varepsilon / k$, for $i=2, \ldots, k$. 
Remark 4. Thanks to (7.6) and to Lemma 24 in Section 10, the integrals in $\mathcal{N}_{\nu ; \boldsymbol{\alpha}, \boldsymbol{\beta}}$ are all absolutely convergent when the line of integration are chosen so that $\Re\left(v_{2}\right)=\cdots=\Re\left(v_{k}\right)=$ $\Re\left(v_{*}\right)=\varepsilon / k$ and $\Re\left(v_{1}\right):=\Re\left(\frac{1}{2}-\alpha_{1}-u_{1}+\frac{w}{2}\right)=B+1-2 \varepsilon$ (and even if an extra factor of $\prod_{i=2}^{k}\left(1+\left|u_{i}\right|+\left|v_{i}\right|\right)^{1+4 \varepsilon}$ is introduced inside the integrals, as will be relevant later on in the argument). In the following computations, until Remark [6, we will (almost) always arrange the lines of integration in a way such that $\Re\left(v_{1}\right)$ is kept equal to $B+1-2 \varepsilon 2$ This ensures the absolute convergence of the integrals in all the bounds we give.

Remark 5. We also observe that, by the definition (10.5), the poles of $\Psi_{\epsilon^{*}, B}\left(v_{1}, \boldsymbol{v}, v_{*}\right)$ are contained in the set

$$
\left\{\left(v_{1}, \boldsymbol{v}, v_{*}\right) \in \mathbb{C}^{k+1} \mid v_{i} \in \mathbb{Z}_{\leq 0} \text { for some } i \in\{1, \ldots, k\} \text { or } v_{1}+\cdots+v_{k}+v_{*}=B+1\right\} \text {. }
$$

\subsection{Picking up the residues of the Estermann function}

For each $i=2, \ldots, k$ we move the line of integration $c_{u_{i}}$ to $c_{u_{i}}=-\frac{1}{2}+\nu_{i}-2 \varepsilon$, passing through the poles of the Estermann function at $u_{i}=\frac{1}{2}-\alpha_{i}-v_{i}+\nu_{i}$ and $u_{i}=\frac{1}{2}-\beta_{i}-v_{i}+\nu_{i}$. By Lemma 2 and the residue theorem, we obtain

$$
\mathcal{N}_{\nu ; \boldsymbol{\alpha}, \boldsymbol{\beta}}=\sum_{\substack{I \cup J=\{2, \ldots, k\}, I \cap J=\emptyset}} \sum_{\substack{\left\{\alpha_{i}^{\prime}, \beta_{i}^{\prime}\right\}=\left\{\alpha_{i}, \beta_{i}\right\} \forall i \in I,\left(\alpha_{j}^{\prime}, \beta_{j}^{\prime}\right)=\left(\alpha_{j}, \beta_{j}\right) \forall j \in J \cup\{1\}}} \mathcal{P}_{I ; \nu ; \boldsymbol{\alpha}^{\prime}, \boldsymbol{\beta}^{\prime}},
$$

where, for $I \cup J=\{2, \ldots, k\}, I \cap J=\emptyset$,

$$
\begin{aligned}
& \mathcal{P}_{I ; \nu ; \boldsymbol{\alpha}, \boldsymbol{\beta}}:=\sum_{d \mid q} \frac{\mu\left(\frac{q}{d}\right)}{\varphi(q)} \sum_{\ell} \frac{P(\ell / L)}{(2 \pi i)^{5}} \int_{\begin{array}{c}
c_{s}, c_{w}, c_{u_{1}} \\
c_{u_{*}, c_{*}}
\end{array}} \frac{q^{k s} d^{1-v_{*}-u_{*}+\nu_{*}}}{\ell^{\sum_{i \in I \cup\{1\}}\left(1-\alpha_{i}+\beta_{i}\right)+w}} \sum_{h(\bmod \ell)}^{*} F\left(v_{*}+u_{*}-\nu_{*}, \pm_{*} \frac{d h}{\ell}\right) \\
& \times\left(\prod_{j \in J} \frac{1}{(2 \pi i)^{2}} \int_{\left(c_{u_{j}}, c_{v_{j}}\right)} D_{\alpha_{j}, \beta_{j}}\left(\frac{1}{2}+u_{j}+v_{j}-\nu_{j}, \frac{ \pm_{j} h}{\ell}\right) \tilde{P}\left(u_{j}-s\right) N_{j}^{u_{j}-s} d u_{j}\right) \\
& \times\left(\prod_{i \in I} \frac{1}{2 \pi i} \int_{\left(c_{i}\right)} \tilde{P}\left(\frac{1}{2}-\alpha_{i}-v_{i}+\nu_{i}-s\right) N_{i}^{\frac{1}{2}-\alpha_{i}-v_{i}+\nu_{i}-s}\right) N_{*}^{u_{*}} \tilde{P}\left(u_{*}\right) N_{1}^{u_{1}-s} \tilde{P}\left(u_{1}-s\right) \\
& \times \Psi_{\epsilon^{*}, B}\left(\frac{1}{2}-\alpha_{1}-u_{1}+\frac{w}{2}, \boldsymbol{v}, v_{*}\right) \frac{H_{I ; \boldsymbol{\alpha}, \boldsymbol{\beta}}^{\prime}(w, s)}{w s} d s d w d u_{1} \boldsymbol{d} \boldsymbol{v} d u_{*} d v_{*}
\end{aligned}
$$

and

$$
H_{I ; \boldsymbol{\alpha}, \boldsymbol{\beta}}^{\prime}(w, s):=G_{\boldsymbol{\alpha}, \boldsymbol{\beta}}(s) G_{\boldsymbol{\alpha}, \boldsymbol{\beta}}(w) g_{\boldsymbol{\alpha}, \boldsymbol{\beta}}(s) \zeta\left(1+w-\alpha_{1}+\beta_{1}\right) \prod_{i \in I} \zeta\left(1-\alpha_{i}+\beta_{i}\right),
$$

\footnotetext{
${ }^{2}$ The only exception is in the proof of $(\sqrt{7.19})$, where we need to take $\Re\left(v_{1}\right)=B-2 \varepsilon$. One can easily verify however that the integrals are all absolutely convergent also in that case.
} 
so that

$$
H_{I ; \boldsymbol{\alpha}, \boldsymbol{\beta}}^{\prime}(w, s) \ll(A \log q)^{|I|} e^{-C_{2}(|\Im(s)|+|\Im(w)|}(1+|\Re(s)|+|\Re(w)|)^{A(|\Re(s)|+|\Re(w)|+k)} .
$$

We remind also that the lines of integrations are given by (7.9) and

$$
c_{u_{j}}=-\frac{1}{2}+\nu_{j}-2 \varepsilon, \forall j \in J
$$

\subsection{Applying the bounds on sums of Kloosterman sums}

In this section, we apply Lemma 8 to give a bound for $\mathcal{P}_{I, \nu ; \boldsymbol{\alpha}, \boldsymbol{\beta}}$ under certain conditions.

Lemma 15. Let $I \subseteq\{2, \ldots, k\}$ and let $J:=\{2, \ldots, k\} \backslash I$. Then, if $|I| \leq|J|$ we have

$$
\mathcal{P}_{I, \nu ; \boldsymbol{\alpha}, \boldsymbol{\beta}} \ll q^{-1+A \varepsilon} N_{1}^{A \varepsilon}\left(q^{\vartheta} N_{1}^{\frac{k+1}{2}}+q^{\frac{k}{2}-\frac{1}{3}+\frac{\vartheta}{3}} N_{1}^{\frac{1}{2}}\right)\left(N_{1} \cdots N_{k}\right)^{-\frac{1}{2}} L^{-\varepsilon},
$$

whereas if $|I|>|J|$ and $\nu_{j}>0$ for some $j \in J$, then

$$
\mathcal{P}_{I, \nu ; \boldsymbol{\alpha}, \boldsymbol{\beta}} \ll q^{-\frac{5}{6}+\frac{\vartheta}{3}+A \varepsilon} N_{1}^{-1+A \varepsilon}\left(N_{1} \cdots N_{k}\right)^{\frac{1}{2}} L^{-\varepsilon} .
$$

Proof. First, we bound the sums over $h$ and $\ell$ by Lemma 8 and we bound trivially the integrals which are all convergent by (15.2), (17.12) and (10.6) when the lines of integrations are given by (7.9) and (7.13). Doing so, we obtain

$$
\begin{aligned}
& \mathcal{P}_{I, \nu ; \boldsymbol{\alpha}, \boldsymbol{\beta}} \ll q^{-\frac{5}{6}+\frac{\vartheta}{3}+A \varepsilon} N_{1}^{-B-\frac{1}{2}+A \varepsilon} N_{*}^{1+\nu_{*}+\varepsilon}\left(\prod_{i \in I} N_{i}^{\frac{1}{2}+\nu_{i}}\right)\left(\prod_{j \in J} N_{j}^{-\frac{1}{2}+\nu_{j}}\right) L^{|J|-|I|-\varepsilon} \times
\end{aligned}
$$

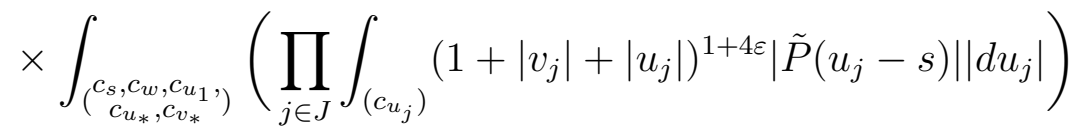

$$
\begin{aligned}
& \times\left(\prod_{i \in I} \int_{\left(c_{v_{i}}\right)}\left|\tilde{P}\left(\frac{1}{2}-\alpha_{i}-v_{i}+\nu_{i}-s\right)\right|\right)\left|\tilde{P}\left(u_{*}\right)\right|\left|\tilde{P}\left(u_{1}-s\right)\right| \\
& \times\left|\Psi_{\epsilon^{*}, B}\left(\frac{1}{2}-\alpha_{1}-u_{1}+\frac{w}{2}, \boldsymbol{v}, v_{*}\right)\right| \frac{\left|H_{I ; \boldsymbol{\alpha}, \boldsymbol{\beta}}^{\prime}(w, s)\right|}{|w s|}\left|d s d w d u_{1} \boldsymbol{d} \boldsymbol{v} d u_{*} d v_{*}\right| \\
& \ll q^{-\frac{5}{6}+\frac{\vartheta}{3}+A \varepsilon} N_{1}^{-B-\frac{1}{2}+A \varepsilon} N_{*}^{1+\nu_{*}}\left(\prod_{i \in I} N_{i}^{\frac{1}{2}+\nu_{i}}\right)\left(\prod_{j \in J} N_{j}^{-\frac{1}{2}+\nu_{j}}\right) L^{|J|-|I|-\varepsilon} .
\end{aligned}
$$

If $|I|-|J|>0$ and at least one of the $\nu_{j}$ is greater than zero, then this is bounded by

$$
\begin{aligned}
\mathcal{P}_{I, \nu ; \boldsymbol{\alpha}, \boldsymbol{\beta}} & \ll q^{-\frac{5}{6}+\frac{\vartheta}{3}+A \varepsilon} N_{1}^{A \varepsilon} \frac{N_{*}^{\nu_{*}+1}}{N_{1}^{\nu_{*}+1}}\left(N_{1} \cdots N_{k}\right)^{\frac{1}{2}}\left(\prod_{i \in I} \frac{N_{i}^{\nu_{i}}}{N_{1}^{\nu_{i}}}\right)\left(\prod_{j \in J} \frac{N_{j}^{\nu_{j}-1}}{N_{1}^{\nu_{j}}}\right) L^{-\varepsilon} \\
& \ll q^{-\frac{5}{6}+\frac{\vartheta}{3}+A \varepsilon} N_{1}^{-1+A \varepsilon}\left(N_{1} \cdots N_{k}\right)^{\frac{1}{2}} L^{-\varepsilon}
\end{aligned}
$$


since $B=\nu_{1}+\cdots+\nu_{k}$ and $N_{2}, \ldots, N_{k} \leq N_{1}, N_{*} \ll k N_{1} q^{\varepsilon / k}$.

Now assume $|I|-|J| \leq 0$ and let $L \leq q^{\frac{1}{2}}$. In this case (7.16) gives

$$
\begin{aligned}
\mathcal{P}_{I, \nu ; \boldsymbol{\alpha}, \boldsymbol{\beta}} & \ll q^{-\frac{5}{6}+\frac{\vartheta}{3}+A \varepsilon} N_{1}^{\frac{1}{2}+|I|+A \varepsilon} \frac{N_{*}^{\nu_{*}+1}}{N_{1}^{\nu_{*}+1}}\left(N_{1} \cdots N_{k}\right)^{-\frac{1}{2}}\left(\prod_{i \in I} \frac{N_{i}^{1+\nu_{i}}}{N_{1}^{1+\nu_{i}}}\right)\left(\prod_{j \in J} \frac{N_{j}^{\nu_{j}}}{N_{1}^{\nu_{j}}}\right) q^{\frac{1}{2}(|J|-|I|)-\varepsilon} \\
& \ll q^{-\frac{5}{6}+\frac{\vartheta}{3}+\frac{1}{2}(|J|-|I|)+A \varepsilon} N_{1}^{\frac{1}{2}+|I|+A \varepsilon}\left(N_{1} \cdots N_{k}\right)^{-\frac{1}{2}} \\
& \ll q^{-\frac{1}{3}+\frac{\vartheta}{3}+A \varepsilon}\left(N_{1}^{\frac{k}{2}+A \varepsilon} q^{-\frac{1}{2}}+q^{\frac{k}{2}-1} N_{1}^{\frac{1}{2}}\right)\left(N_{1} \cdots N_{k}\right)^{-\frac{1}{2}}
\end{aligned}
$$

since $|I|=k-1-|J|$ and $\frac{k-1}{2} \leq|J| \leq k-1$.

Finally, if $|I|-|J| \leq 0$ and $L>q^{\frac{1}{2}}$, then we move the lines of integration $c_{w}$ and $c_{u_{1}}$ to

$$
c_{w}=|J|-|I|+10 \varepsilon=k-1-2|I|+10 \varepsilon, \quad c_{u_{1}}=-1-B+\frac{k}{2}-\Re\left(\alpha_{1}\right)-|I|+7 \varepsilon .
$$

Then, we use Lemma 8 and bound trivially the integrals (using (5.2), (7.12) and (10.6)) and we obtain

$$
\begin{aligned}
\mathcal{P}_{I, \nu ; \boldsymbol{\alpha}, \boldsymbol{\beta}} & \ll q^{-1+\vartheta+A \varepsilon} N_{1}^{-1-B+\frac{k}{2}-|I|+A \varepsilon} N_{*}^{1+\nu_{*}}\left(\prod_{i \in I} N_{i}^{\frac{1}{2}+\nu_{i}}\right)\left(\prod_{j \in J} N_{j}^{-\frac{1}{2}+\nu_{j}}\right) L^{-\varepsilon} \\
& \ll \frac{q^{-1+\vartheta+A \varepsilon}}{L^{\varepsilon}} N_{1}^{\frac{k}{2}+A \varepsilon} \frac{N_{*}^{\nu_{*}+1}}{N_{1}^{\nu_{*}+1}}\left(\prod_{i \in I} \frac{N_{i}}{N_{1}}\right)\left(\prod_{i=2}^{k} \frac{N_{i}^{-\frac{1}{2}+\nu_{i}}}{N_{1}^{\nu_{i}}}\right) \ll \frac{q^{-1+\vartheta+A \varepsilon} N_{1}^{\frac{k}{2}+\frac{1}{2}+A \varepsilon}}{\left(N_{1} \cdots N_{k}\right)^{\frac{1}{2}} L^{\varepsilon}} .
\end{aligned}
$$

Thus, since $N_{1}^{\frac{k}{2}} q^{-\frac{1}{2}} \ll q^{\frac{k}{2}-1} N_{1}^{\frac{1}{2}}+q^{-1} N_{1}^{\frac{k}{2}+\frac{1}{2}}$, we have that (7.17) and (7.18) imply (7.14).

\subsection{Reassembling the sum over $\nu$ and further manipulations}

By the previous section, we only need to consider the $\mathcal{P}_{I ; \nu ; \boldsymbol{\alpha}, \boldsymbol{\beta}}$ with $|I|>|J|$ and $\nu_{j}=0$ for all $j \in J$ (and lines of integration given (7.9) and (7.13)). For each $j \in J$, we move $c_{u_{j}}$ to $\frac{1}{2}+\nu_{j}-2 \varepsilon$ and contextually $c_{v_{j}}$ to $c_{v_{j}}=-1+\varepsilon / k$, passing through the pole of $\Psi_{\epsilon^{*}, B}$ at $v_{j}=0$. The contribution of the integral on the new line of integration can be bounded by

$$
\ll q^{-\frac{5}{6}+\frac{\vartheta}{3}+A \varepsilon} N_{1}^{-1+A \varepsilon}\left(N_{1} \cdots N_{k}\right)^{\frac{1}{2}} L^{-\varepsilon},
$$

as can be see by moving $c_{u_{1}}$ to $c_{u_{1}}=-B-\frac{3}{2}-\alpha_{1}+7 \varepsilon$ and bounding the sums and integrals as in the proof of (7.15). Thus we only need to consider the residue at $v_{j}=0$ for all $j \in J$.

In the same way, we move the line of integration $c_{v_{*}}$ to $c_{v_{*}}=1+\varepsilon / k$ and $c_{u_{*}}$ to $c_{u_{*}}=$ $\nu_{*}+2 \varepsilon-\varepsilon / k$, passing through the pole of $\Psi_{\epsilon^{*}, B}$ at $v_{*}=B+1-\left(\frac{1}{2}-\alpha_{1}-u_{1}+\frac{w}{2}\right)-\sum_{i \in I} v_{i}$. The contribution of the new line of integration can be bounded by (7.19) in a similar way, 
so again we only need to consider the contribution of the residue. Thus, summarizing (and recalling (7.7) and (17.10) $)$, we arrive to

$$
\begin{aligned}
\mathcal{L}_{\boldsymbol{\alpha}, \boldsymbol{\beta}}= & \sum_{\substack{I \cup J=\{2, \ldots, k\}, I \cap J=\emptyset,|I|>|J|}} \sum_{\substack{\left\{\alpha_{i}^{\prime}, \beta_{i}^{\prime}\right\}=\left\{\alpha_{i}^{\prime}, \beta_{j}^{\prime}\right\}=\left(\alpha_{j}, \beta_{j}\right) \forall j \in J \cup\{1\} \\
\forall i \in I,}} \sum_{\nu} \frac{B !}{\nu_{2} ! \cdots \nu_{k} ! \nu_{*} !} \mathcal{Q}_{I ; \nu ; \boldsymbol{\alpha}, \boldsymbol{\beta}} \\
& +O\left(\frac{q^{-1+A \varepsilon} N_{1}^{A \varepsilon}}{\left(N_{1} \cdots N_{k}\right)^{\frac{1}{2}}}\left(q^{\vartheta} N_{1}^{\frac{k+1}{2}}+q^{\frac{k}{2}-\frac{1}{3}+\frac{\vartheta}{3}} N_{1}^{\frac{1}{2}}+q^{\frac{1}{6}+\frac{\vartheta}{3}} N_{2} \cdots N_{k}\right) L^{-\varepsilon}\right),
\end{aligned}
$$

where the sum over $\nu$ is now over $\nu=\left(\nu_{2}, \ldots, \nu_{k}, \nu_{*}\right) \in \mathbb{Z}_{\geq 0}^{k}$ satisfying

$$
\nu_{2}+\cdots+\nu_{k}+\nu_{*}=B, \quad \nu_{i}=0 \text { if } \pm_{i} 1=-1 \text { or } i \in J, \quad \nu_{*}=0 \text { if } \pm_{*} 1=-1
$$

and where

$$
\begin{aligned}
& \mathcal{Q}_{I ; \nu ; \boldsymbol{\alpha}, \boldsymbol{\beta}}:=\sum_{d \mid q} \frac{\mu\left(\frac{q}{d}\right)}{\varphi(q)} \sum_{\ell} \sum_{h(\bmod \ell)}^{*} \frac{P(\ell / L)}{(2 \pi i)^{4+|I|}} \int_{\substack{\left.c_{s}, c_{w}, c_{u_{1}}, c_{u_{*}}, c_{v_{i}} \forall i \in I\right)}} \frac{d^{1-\left(B+\frac{1}{2}+\alpha_{1}+u_{1}-\frac{w}{2}\right)-u_{*}+\nu_{*}}}{\ell^{\sum_{i \in I \cup\{1\}}\left(1-\alpha_{i}+\beta_{i}\right)+w}} \\
& \times q^{k s} F\left(B+\frac{1}{2}+\alpha_{1}+u_{1}-\frac{w}{2}-\sum_{i \in I} v_{i}+u_{*}-\nu_{*}, \pm_{*} \frac{d h}{\ell}\right) \frac{H_{I ; \boldsymbol{\alpha}, \boldsymbol{\beta}}^{\prime}(w, s)}{w s} \\
& \times\left(\prod_{j \in J} \frac{1}{2 \pi i} \int_{\left(c_{u_{j}}\right)} D_{\alpha_{j}, \beta_{j}}\left(\frac{1}{2}+u_{j}, \frac{ \pm_{j} h}{\ell}\right) \tilde{P}\left(u_{j}-s\right) N_{j}^{u_{j}-s} d u_{j}\right) \\
& \times N_{*}^{u_{*}} \tilde{P}\left(u_{*}\right) N_{1}^{u_{1}-s} \tilde{P}\left(u_{1}-s\right) \Psi_{I ; \epsilon_{I}^{*}, B}^{\prime}\left(\frac{1}{2}-\alpha_{1}-u_{1}+\frac{w}{2}, \boldsymbol{v}_{I}\right) \\
& \times\left(\prod_{i \in I} \tilde{P}\left(\frac{1}{2}-\alpha_{i}-v_{i}+\nu_{i}-s\right) N_{i}^{\frac{1}{2}-\alpha_{i}-v_{i}+\nu_{i}-s} d^{v_{i}} d v_{i}\right) d w d u_{1} d s d u_{*},
\end{aligned}
$$

for $\boldsymbol{v}_{I}:=\left(v_{i}\right)_{i \in I}, \epsilon_{I}^{*}=\left( \pm_{i} 1\right)_{i \in I \cup\{*\}}$ and

$$
\begin{aligned}
\Psi_{I ; \epsilon_{I}^{*}, B}^{\prime}\left(v_{1}, \boldsymbol{v}_{I}\right) & :=\frac{\Gamma\left(B+1-v_{1}-\sum_{i \in I} v_{i}\right) \prod_{i \in I \cup\{1\}} \Gamma\left(v_{i}\right)}{\Gamma\left(V_{\mp_{*} ; \epsilon_{I}}\left(v_{1}, \boldsymbol{v}_{I}\right)\right) \Gamma\left(B+1-V_{\mp_{*} ; \epsilon_{I}}\left(v_{1}, \boldsymbol{v}_{I}\right)\right)}, \\
V_{ \pm, \epsilon_{I}}\left(v_{1}, \boldsymbol{v}_{I}\right) & :=\sum_{\substack{i \in I \cup\{1\}, \pm_{i} 1= \pm 1}} v_{i} .
\end{aligned}
$$

We also remind that the line of integrations are

$$
\begin{aligned}
& c_{s}:=\varepsilon / k, \quad c_{w}=10 \varepsilon, \quad c_{u_{*}}=1+\nu_{*}+2 \varepsilon-\varepsilon / k, \\
& c_{u_{j}}=-\frac{1}{2}+\nu_{j}-2 \varepsilon \forall j \in J, \quad c_{v_{i}}=\frac{\varepsilon}{k} \forall i \in I
\end{aligned}
$$

and $c_{u_{1}}=-B-\frac{1}{2}-\Re\left(\alpha_{1}\right)+7 \varepsilon$. 
Remark 6. Notice that the integrand in (7.21) decays rapidly along vertical strip in each of the variables of integration. In particular, in the following computations we will always be able to bound the integrals trivially.

At this point, we wish to execute the sum over the partitions of unity $N_{*}$. However, first we need to remove the truncation $N_{*} \ll k N_{1} q^{\varepsilon / k}$. This can be done at a negligible cost, as it is shown in the following lemma.

Lemma 16. For $N_{*} \gg k N_{1} q^{\varepsilon}$ we have

$$
\sum_{N_{*} \ll k N_{1} q^{\varepsilon / k}}^{\dagger} \mathcal{Q}_{I ; \nu ; \boldsymbol{\alpha}, \boldsymbol{\beta}}=\sum_{N_{*}}^{\dagger} \mathcal{Q}_{I ; \nu ; \boldsymbol{\alpha}, \boldsymbol{\beta}}+O\left(q^{-2+A \varepsilon} N_{1}^{A \varepsilon}\left(N_{1} \cdots N_{k}\right)^{\frac{1}{2}} L^{-\varepsilon}\right) .
$$

Proof. Given a large positive integer $\Delta$, we move $c_{v_{i}}$ to $c_{v_{i}}=-\Delta k+\frac{1}{2}$ for all $i \in I$. Doing so, we pass through the poles of $\Psi_{I ; \epsilon_{I}^{*}, B}^{\prime}\left(\frac{1}{2}-\alpha_{1}-u_{1}+\frac{w}{2}, \boldsymbol{v}_{I}\right)$ at $v_{i} \in S_{\Delta}:=\{-r \mid r \in \mathbb{Z}, 0 \leq r<$ $\Delta k\}$, so that we have to deal with a sum of $(\Delta k+1)^{|I|}$ terms coming from the contribution of the residues and of the integrals in the new line of integrations 3 Then, for each of these terms, we move the line of integration $c_{u_{1}}$ to $c_{u_{1}}=\Delta k$. This can be done without crossing any pole of $\Psi_{I ; \epsilon_{I}^{*}, B}^{\prime}$ if the term was coming from picking up a residue in each of the variables $v_{i}$ for all $i \in I$, since in this case the $\Gamma$ factor in the denominator of $\Psi_{I ; \epsilon_{I}^{*}, B}^{\prime}$ cancel the poles of $\Gamma\left(v_{1}\right)=\Gamma\left(\frac{1}{2}-\alpha_{1}-u_{1}+\frac{w}{2}\right)$. Otherwise, we also have to consider the residues of $\Psi_{I ; \epsilon_{I}^{*}, B}^{\prime}$ at $\frac{1}{2}-\alpha_{1}-u_{1}+\frac{w}{2} \in S_{\Delta}$. In all cases, however, all the terms will still have at least one integral left (besides the $w$ and $u_{*}$ integrals) with line of integration $c_{v_{i}}=-\Delta k+\frac{1}{2}$ or $c_{u_{1}}=\Delta k$. Finally, for each of these terms we place the line of integration $c_{u_{*}}$ so that the real part of the argument of the function $F$ in (7.21) is still $1+(4-|I| / k) \varepsilon$ (we can do this without crossing poles).

Thus, bounding these terms by using Lemma 8 and the estimates (5.2), (7.12) and (10.6), we obtain

$$
\begin{aligned}
\mathcal{Q}_{I ; \nu ; \boldsymbol{\alpha}, \boldsymbol{\beta}} & \ll q^{-\frac{5}{6}+\frac{\vartheta}{3}+A \varepsilon} \sum_{\substack{r_{1}, \ldots r_{k} \in\left(\{0,1, \ldots, \Delta-1\} \cup\left\{\Delta k-\frac{1}{2}\right\}\right), r_{i}=\Delta k-\frac{1}{2} \text { for some } i \in(\{1\} \cup I), r_{i}=0 \text { if } i \in J}} \frac{N_{1}^{\frac{1}{2}+r_{1}+A \varepsilon} N_{2}^{r_{2}+\frac{1}{2}+\nu_{2}} \cdots N_{k}^{r_{k}+\frac{1}{2}+\nu_{k}}}{N_{*}^{B+\sum_{i=1}^{k} r_{i}-\nu_{*}+(4-|I| / k) \varepsilon} L^{\varepsilon}} \prod_{j \in J} N_{j}^{-1} \\
& \ll q^{-1+A \varepsilon} \frac{N_{1}^{A \varepsilon}\left(N_{1} \cdots N_{k}\right)^{\frac{1}{2}}}{q^{\Delta \varepsilon} N_{*}^{\varepsilon}}\left(\prod_{j \in J} N_{j}^{-1}\right) L^{-\varepsilon} \ll q^{-2+A \varepsilon} N_{1}^{A \varepsilon}\left(N_{1} \cdots N_{k}\right)^{\frac{1}{2}} L^{-\varepsilon},
\end{aligned}
$$

if $\Delta$ is large enough with respect to $\varepsilon$. Equation (7.24) then follows.

\footnotetext{
${ }^{3}$ In total there are $(\Delta k+1)^{|I|}$ terms because for each $v_{i}$ we have the possibility of taking the residue at $v_{i}=-r_{i} \in S_{\Delta}$ or to take the integral at $c_{v_{i}}=-\Delta k+\frac{1}{2}$.
} 
We now move the line of integration $c_{u_{1}}$ to $c_{u_{1}}=\frac{1}{2}+3 \varepsilon$ and then execute the sum over $N_{*}$, which we do by using the following Lemma whose (simple) proof is left to the reader.

Lemma 17. Let $K(s)$ be a function which is analytic and grows at most polynomially on a strip $|\Re(s)|<c$ for some $c>0$. Then, for any $-c<c_{u}<c$ we have

$$
\sum_{N}^{\dagger} \frac{1}{2 \pi i} \int_{(c)} K(u) \tilde{P}(u) N^{u} d u=K(0) .
$$

We move the line of integration $c_{u_{1}}$ to $c_{u_{1}}=\frac{1}{2}+3 \varepsilon$ without crossing poles and apply the above lemma obtaining

$$
\begin{aligned}
\sum_{N_{*}}^{\dagger} \mathcal{Q}_{I ; \nu ; \boldsymbol{\alpha}, \boldsymbol{\beta}}= & \sum_{d \mid q} \frac{\mu\left(\frac{q}{d}\right)}{\varphi(q)} \sum_{\ell} \sum_{h(\bmod \ell)}^{*} \frac{P(\ell / L)}{(2 \pi i)^{3+|I|}} \int_{\begin{array}{c}
\left.c_{c_{s}, c_{w},}, c_{v_{i}} \forall i \in I\right) \\
c_{u_{1}},{ }^{k}
\end{array}} \frac{q^{k s} d^{1-\left(B+\frac{1}{2}+\alpha_{1}+u_{1}-\frac{w}{2}\right)+\nu_{*}}}{\ell^{\sum_{i \in I \cup\{1\}}\left(1-\alpha_{i}+\beta_{i}\right)+w}} \\
& \times F\left(B+\frac{1}{2}+\alpha_{1}+u_{1}-\frac{w}{2}-\sum_{i \in I} v_{i}-\nu_{*}, \pm_{*} \frac{h}{\ell}\right) \\
& \times\left(\prod_{j \in J} \frac{1}{2 \pi i} \int_{\left(c_{u_{j}}\right)} D_{\alpha_{j}, \beta_{j}}\left(\frac{1}{2}+u_{j}, \frac{ \pm_{j} h}{\ell}\right) \tilde{P}\left(u_{j}-s\right) N_{j}^{u_{j}-s} d u_{j}\right) \\
& \times N_{1}^{u_{1}-s} \tilde{P}\left(u_{1}-s\right) \Psi_{I ; \epsilon_{I}^{*}, B}^{\prime}\left(\frac{1}{2}-\alpha_{1}-u_{1}+\frac{w}{2}, \boldsymbol{v}_{I}\right) \frac{H_{I ; \boldsymbol{\alpha}, \boldsymbol{\beta}}^{\prime}(w, s)}{w s} \\
& \times \prod_{i \in I}\left(\tilde{P}\left(\frac{1}{2}-\alpha_{i}-v_{i}+\nu_{i}-s\right) N_{i}^{\frac{1}{2}-\alpha_{i}-v_{i}+\nu_{i}-s} d^{v_{i}} \mathrm{~d} v_{i}\right) \mathrm{d} w \mathrm{~d} u_{1} \mathrm{~d} s
\end{aligned}
$$

with lines of integrations that we can take to be given by (7.23) and $c_{u_{1}}=\frac{1}{2}+3 \varepsilon$.

We are finally ready to execute the sum over $\nu$. We do this in the following Lemma, which also summarizes the previous computations.

Lemma 18. We have

$$
\begin{aligned}
\sum_{\epsilon \in\{ \pm 1\}^{k}} \rho_{\Upsilon}(\epsilon) \mathcal{O}_{\epsilon, \alpha, \beta}^{\prime \prime}= & 2 \sum_{\substack{L \cup J=\{2, \ldots, k\}, I \cap J=\emptyset,|I|>|J|}}^{\dagger} \sum_{\substack{\left\{\alpha_{i}^{\prime}, \beta_{i}^{\prime}\right\}=\left\{\alpha_{i}, \beta_{i}\right\} \forall i \in I_{1},\left(\alpha_{j}^{\prime}, \beta_{j}^{\prime}\right)=\left(\alpha_{j}, \beta_{j}\right) \forall j \in J}} \sum_{\substack{\epsilon \in\{ \pm 1\}^{k}, \pm 1 \\
\pm 1=-1}} \rho_{\Upsilon}(\epsilon) \sum_{ \pm * 1 \in\{ \pm 1\}} \mathcal{R}_{I ; \epsilon^{*} ; \boldsymbol{\alpha}, \boldsymbol{\beta}} \\
& +O\left(\frac{q^{-1+A \varepsilon} N_{1}^{A \varepsilon}}{\left(N_{1} \cdots N_{k}\right)^{\frac{1}{2}} L^{\varepsilon}}\left(q^{\vartheta} N_{1}^{\frac{k+1}{2}}+q^{\frac{k}{2}-\frac{1}{3}+\frac{\vartheta}{3}} N_{1}^{\frac{1}{2}}+q^{\frac{1}{6}+\frac{\vartheta}{3}} N_{2} \cdots N_{k}\right)\right)
\end{aligned}
$$


where $I_{1}:=I \cup\{1\}$ and

$$
\begin{aligned}
\mathcal{R}_{I ; \epsilon^{*} ; \boldsymbol{\alpha}, \boldsymbol{\beta}}:= & \sum_{d \mid q} \frac{\mu\left(\frac{q}{d}\right)}{\varphi(q)} \sum_{\ell} \sum_{h(\bmod \ell)}^{*} \frac{P(\ell / L)}{(2 \pi i)^{3+|I|}} \int_{\begin{array}{c}
\left.c_{c_{s}, c_{w},} c_{v_{i}} \forall i \in I\right) \\
c_{u_{1}},
\end{array}} \frac{q^{k s} d^{\frac{1}{2}-\alpha_{1}-u_{1}+\frac{w}{2}} N_{1}^{u_{1}-s}}{\ell^{\sum_{i \in I_{1}}\left(1-\alpha_{i}+\beta_{i}\right)+w}} \\
& \times\left(\prod_{j \in J} \frac{1}{2 \pi i} \int_{\left(c_{u_{j}}\right)} D_{\alpha_{j}, \beta_{j}}\left(\frac{1}{2}+u_{j}, \frac{ \pm_{j} h}{\ell}\right) \tilde{P}\left(u_{j}-s\right) N_{j}^{u_{j}-s} d u_{j}\right) \\
& \tilde{P}\left(u_{1}-s\right) \times F\left(\frac{1}{2}+\alpha_{1}+u_{1}-\frac{w}{2}-\sum_{i \in I} v_{i}, \pm_{*} \frac{d h}{\ell}\right) \Psi_{I ; \epsilon_{I}^{*}, 0}^{\prime}\left(\frac{1}{2}-\alpha_{1}-u_{1}+\frac{w}{2}, \boldsymbol{v}_{I}\right) \\
& \times\left(\prod_{i \in I} \tilde{P}\left(\frac{1}{2}-\alpha_{i}-v_{i}-s\right) N_{i}^{\frac{1}{2}-\alpha_{i}-v_{i}-s} d^{v_{i}} d v_{i}\right) \frac{H_{I ; \boldsymbol{\alpha}, \boldsymbol{\beta}}^{\prime}(w, s)}{w s} d w d u_{1} d s
\end{aligned}
$$

and lines of integrations given by (7.23) and $c_{u_{1}}=\frac{1}{2}+3 \varepsilon$.

Proof. Using (7.1), (17.4), (7.20) and (7.24) we obtain (17.26), with $\mathcal{R}_{I ; \epsilon^{*} ; \boldsymbol{\alpha}, \boldsymbol{\beta}}$ replaced by

$$
\mathcal{R}_{I ; \epsilon^{*} ; \boldsymbol{\alpha}, \boldsymbol{\beta}}^{\prime}:=\sum_{\nu} \frac{B !}{\nu_{2} ! \cdots \nu_{k} ! \nu_{*} !} \sum_{N_{*}}^{\dagger} \mathcal{Q}_{I ; \nu ; \boldsymbol{\alpha}, \boldsymbol{\beta}}
$$

and $\sum^{\dagger} \mathcal{Q}_{I ; \nu ; \boldsymbol{\alpha}, \boldsymbol{\beta}}$ as in (7.25). Thus, the Lemma reduces to showing that $\mathcal{R}_{I ; \epsilon^{*} ; \boldsymbol{\alpha}, \boldsymbol{\beta}}=\mathcal{R}_{I ; \epsilon^{*} ; \boldsymbol{\alpha}, \boldsymbol{\beta}}^{\prime}$. This is an immediate consequence of Lemma 25 below, which is applicable since the pole of $F$ is canceled by the sum over $d$.

\subsection{Reassembling the sum over $\epsilon$}

Now, we can also get rid of the integral over $w$. To do this, first we move the lines of integration $c_{u_{1}}$ and $c_{u_{j}}$ for $j \in J$ (without passing through poles), so that we have

$$
c_{u_{1}}=-\Re\left(\alpha_{1}\right)+7 \varepsilon, \quad c_{u_{j}}=\frac{1}{2}-\Re\left(\alpha_{j}\right)-2 \varepsilon \forall j \in J \quad c_{s}:=\varepsilon / k, \quad c_{v_{i}}=\frac{\varepsilon}{k} \forall i \in I
$$

and then we move $c_{w}$ to $c_{w}=-1+10 \varepsilon$ passing through a pole at $w=0$. The contribution of the new line of integration is trivially bounded by

$$
\ll q^{-1+A \varepsilon} N_{1}^{-\frac{1}{2}+A \varepsilon}\left(N_{1} \cdots N_{k}\right)^{\frac{1}{2}} L^{-\varepsilon} .
$$

since we have the convexity bound $D\left(1-2 \varepsilon+i t, \alpha_{j}-\beta_{j}, \frac{h}{\ell}\right) \ll \ell^{3 \varepsilon}(1+|t|)^{3 \varepsilon}$ and $|I| \geq 2$ (since $|I|>|J|$ and $k \geq 3$ ). Thus, we only need to consider the contribution from the residue at $w=0$. 
By the convexity bound $F\left(\frac{1}{2}+7 \varepsilon-|I| \varepsilon / k+i t, \frac{h}{\ell}\right) \ll \ell^{\frac{1}{2}}(1+|t|)^{\frac{1}{2}}$, the contribution of the $d=1$ term is also bounded by (7.28). Thus, using also that $\varphi(q)^{-1}=q^{-1}+O\left(q^{-2}\right)$ for $q$ prime, we have

$$
\mathcal{R}_{I ; \epsilon^{*} ; \boldsymbol{\alpha}, \boldsymbol{\beta}}=\mathcal{S}_{I ; \epsilon^{*} ; \boldsymbol{\alpha}, \boldsymbol{\beta}}+O\left(q^{-1+A \varepsilon} N_{1}^{-\frac{1}{2}+A \varepsilon}\left(N_{1} \cdots N_{k}\right)^{\frac{1}{2}} L^{-\varepsilon}\right)
$$

with

$$
\begin{aligned}
\mathcal{S}_{I ; \epsilon^{*} ; \boldsymbol{\alpha}, \boldsymbol{\beta}}= & \sum_{\ell} \sum_{h(\bmod \ell)}^{*} \frac{P(\ell / L)}{(2 \pi i)^{2+|I|}} \int_{\begin{array}{c}
c_{s}, \\
\left.c_{u_{1}}, c_{v_{i}} \forall i \in I\right)
\end{array}} \frac{q^{k s-\frac{1}{2}-\alpha_{1}-u_{1}}}{\ell^{\sum_{i \in I \cup\{1\}}\left(1-\alpha_{i}+\beta_{i}\right)}} N_{1}^{u_{1}-s} \tilde{P}\left(u_{1}-s\right) \\
& \times\left(\prod_{j \in J} \frac{1}{2 \pi i} \int_{\left(c_{u_{j}}\right)} D_{\alpha_{j}, \beta_{j}}\left(\frac{1}{2}+u_{j}, \pm_{*} \frac{ \pm_{j} h}{\ell}\right) \tilde{P}\left(u_{j}-s\right) N_{j}^{u_{j}-s} d u_{j}\right) \\
& \times F\left(\frac{1}{2}+\alpha_{1}+u_{1}-\sum_{i \in I} v_{i}, \frac{q h}{\ell}\right) \Psi_{I ; \epsilon_{I}^{*}, 0}^{\prime}\left(\frac{1}{2}-\alpha_{1}-u_{1}, \boldsymbol{v}_{I}\right) \\
& \times\left(\prod_{i \in I} \tilde{P}\left(\frac{1}{2}-\alpha_{i}-v_{i}-s\right) N_{i}^{\frac{1}{2}-\alpha_{i}-v_{i}-s} q^{v_{i}} d v_{i}\right) \frac{H_{I ; \boldsymbol{\alpha}, \boldsymbol{\beta}}^{\prime}(0, s)}{s} d u_{1} d s
\end{aligned}
$$

and lines of integration given by (7.27). Notice that we made the change of variable $h \rightarrow \pm_{*} h$.

We are ready to reassemble the sum over $\epsilon$. To do this, first we split $\epsilon$ into $\epsilon_{I_{1}}$ and $\epsilon_{J}$, where $\epsilon_{S}:=\left( \pm_{i} 1\right)_{i \in S}$; in particular, $\rho_{\Upsilon}(\epsilon)=\rho_{\Upsilon}\left(\epsilon_{I_{1}}\right) \rho_{\Upsilon}\left(\epsilon_{J}\right)$ where, with a slight abuse of notation, we write $\rho_{\Upsilon}\left(\epsilon_{S}\right):=\prod_{i \in \Upsilon \cap S}\left( \pm_{i} 1\right)$. Then, we observe that

$$
\sum_{\epsilon_{J} \in\{ \pm 1\}|J|} \rho_{\Upsilon}\left(\epsilon_{J}\right) \prod_{j \in J} D_{\alpha_{j}, \beta_{j}}\left(s_{j}, \pm_{*} \frac{ \pm_{j} h}{\ell}\right)=2^{|J|}\left( \pm_{*} i\right)^{|\Upsilon \cap J|} \prod_{j \in J} D_{j ; \alpha_{j}, \beta_{j}}\left(s_{j}, \frac{h}{\ell}\right) .
$$

Thus,

$$
\begin{aligned}
& \sum_{\substack{\epsilon \in\{ \pm 1\}^{k}, \pm 11=-1}} \rho_{\Upsilon}(\epsilon) \sum_{ \pm * 1 \in\{ \pm 1\}} \mathcal{S}_{I ; \epsilon^{*} ; \boldsymbol{\alpha}, \boldsymbol{\beta}}=2^{|J|} i^{|\Upsilon \cap J|} \sum_{\ell} \sum_{h(\bmod \ell)}^{*} \frac{P(\ell / L)}{(2 \pi i)^{2+|I|}} \frac{q^{k s-\frac{1}{2}-\alpha_{1}-u_{1}}}{\ell^{\sum_{i \in I \cup\{1\}}\left(1-\alpha_{i}+\beta_{i}\right)}} \\
& \quad \times \int_{\substack{\left.c_{c_{s}}, c_{v_{i}} \forall i \in I\right) \\
\quad}}\left(\prod_{j \in J} \frac{1}{2 \pi i} \int_{\left(c_{u_{j}}\right)} D_{j ; \alpha_{j}, \beta_{j}}\left(\frac{1}{2}+u_{j}, \frac{h}{\ell}\right) \tilde{P}\left(u_{j}-s\right) N_{j}^{u_{j}-s} d u_{j}\right) \\
& \quad \times N_{1}^{u_{1}-s} \tilde{P}\left(u_{1}-s\right) F\left(\frac{1}{2}+\alpha_{1}+u_{1}-\sum_{i \in I} v_{i}, \frac{q h}{\ell}\right) \mathcal{X}_{I}\left(\frac{1}{2}-u_{1}-\alpha_{1}, \boldsymbol{v}_{I}\right) \\
& \quad \times\left(\prod_{i \in I} \tilde{P}\left(\frac{1}{2}-\alpha_{i}-v_{i}-s\right) N_{i}^{\frac{1}{2}-\alpha_{i}-v_{i}-s} q^{v_{i}} d v_{i}\right) \frac{H_{I ; \boldsymbol{\alpha}, \boldsymbol{\beta}}^{\prime}(0, s)}{s} d u_{1} d s
\end{aligned}
$$


with

$$
\begin{aligned}
& \mathcal{X}_{I}\left(v_{1}, \boldsymbol{v}_{I}\right):=\sum_{ \pm_{*} \in\{ \pm 1\}}\left( \pm_{*} 1\right)^{|\Upsilon \cap J|} \sum_{\substack{\epsilon_{I_{1}} \in\{ \pm 1\}\left|I_{1}\right|, \pm_{1} 1=-1}} \rho_{\Upsilon}\left(\epsilon_{I_{1}}\right) \Psi_{I ; \epsilon_{I}^{*}, 0}^{\prime}\left(v_{1}, \boldsymbol{v}_{I}\right), \\
& =\Gamma\left(1-\sum_{i \in I_{1}} v_{i}\right) \prod_{i \in I_{1}} \Gamma\left(v_{i}\right) \sum_{ \pm_{*} 1 \in\{ \pm 1\}}\left( \pm_{*} 1\right)^{\left|\Upsilon \cap \cap I_{1}\right|} \\
& \times \sum_{\substack{\epsilon_{I_{1}} \in\{ \pm 1\}\left|I_{1}\right|, \pm_{1} 1=-1}} \rho_{\Upsilon}\left(\epsilon_{I_{1}}\right)\left(\Gamma\left(\sum_{\substack{i \in I_{1}, \pm_{i} 1=\mathcal{F}_{*} 1}} v_{i}\right) \Gamma\left(1-\sum_{\substack{i \in I_{1}, \pm_{i} 1=\mathcal{F}_{*} 1}} v_{i}\right)\right)^{-1}
\end{aligned}
$$

by the definition (17.22) of $\Psi_{I ; \epsilon_{I}^{*}, 0}^{\prime}$ and since $\left( \pm_{*} 1\right)^{|\Upsilon \cap J|}=\left( \pm_{*} 1\right)^{|\Upsilon \cap(I \cup\{1\})|}$ for $|\Upsilon|$ even (as we have assumed). Also, we remind that we defined $\epsilon_{I}^{*}:=\left( \pm_{i} 1\right)_{i \in I \cup\{*\}}$ and $I_{1}:=I \cup\{1\}$.

We will now give a $\Gamma$-function identity, which we will use to give a symmetric expression for $\mathcal{X}_{I}\left(v_{1}, \boldsymbol{v}_{I}\right)$.

Lemma 19. Let $r \geq 1, \Theta \subseteq\{1, \ldots, r\}$ and $\left(s_{1}, \ldots, s_{r}\right) \in \mathbb{C}^{r}$. For $\epsilon_{r}=\left( \pm_{1}, \ldots, \pm_{r} 1\right) \in\{ \pm 1\}^{r}$ let $\rho_{\Theta}(\epsilon):=\prod_{i \in \Theta}\left( \pm_{i} 1\right)$. Then, $\rfloor^{4}$

$$
\begin{aligned}
& \prod_{i=1}^{r} \Gamma\left(s_{i}\right) \sum_{ \pm_{*} 1 \in\{ \pm 1\}}\left( \pm_{*} 1\right)^{|\Theta|} \sum_{\substack{\epsilon \in\{ \pm 1\}^{r} \\
\pm_{1} 1=-1}} \rho_{\Theta}(\epsilon)\left(\Gamma\left(\sum_{ \pm_{i} 1=\mp * 1} s_{i}\right) \Gamma\left(1-\sum_{ \pm_{i} 1=\mp * 1} s_{i}\right)\right)^{-1} \\
& \quad=\frac{2^{s_{1}+\cdots+s_{r}}}{\pi^{1-\frac{r}{2}}}\left(\prod_{i \in \Theta} \frac{\Gamma\left(\frac{1}{2}+\frac{s_{i}}{2}\right)}{\Gamma\left(1-\frac{s_{i}}{2}\right)}\right)\left(\prod_{i \notin \Theta} \frac{\Gamma\left(\frac{s_{i}}{2}\right)}{\Gamma\left(\frac{1}{2}-\frac{s_{i}}{2}\right)}\right) \sin \left(\frac{\pi}{2}\left(s_{1}+\cdots+s_{r}\right)-\frac{\pi}{2}|\Theta|\right) .
\end{aligned}
$$

Proof. First, we observe that, by analytic continuation, we can assume that $s_{1}, \ldots, s_{r} \in \mathbb{R} \backslash \mathbb{Z}$. Thus, using the reflection formula for the Gamma function to have

$$
\left(\Gamma\left(\sum_{ \pm_{i} 1=\mp * 1} s_{i}\right) \Gamma\left(1-\sum_{ \pm_{i} 1=\mp_{*} 1} s_{i}\right)\right)^{-1}=\pi^{-1} \sin \left(\pi \sum_{ \pm_{i} 1=\mp_{*} 1} s_{i}\right)=\pi^{-1} \Im\left(\exp \left(\pi i \sum_{ \pm_{i} 1=\mp * 1} s_{i}\right)\right) .
$$

It follows that

$$
\begin{aligned}
S & :=\sum_{ \pm_{*} 1 \in\{ \pm 1\}}\left( \pm_{*} 1\right)^{|\Theta|} \sum_{\substack{\epsilon \in\{ \pm 1\}^{r}, \pm 11=-1}} \rho_{\Theta}(\epsilon)\left(\Gamma\left(\sum_{ \pm_{i} 1=\mp_{*} 1} s_{i}\right) \Gamma\left(1-\sum_{ \pm_{i} 1=\mp_{*} 1} s_{i}\right)\right)^{-1} \\
& =\pi^{-1} \Im\left(e^{\pi i s_{1}} A_{+}+(-1)^{|\Theta|} A_{-}\right),
\end{aligned}
$$

\footnotetext{
${ }^{4}$ The identity has to be interpreted as an identity between meromorphic functions.
} 
where

$$
A_{ \pm}:=\sum_{\substack{\epsilon \in\{ \pm 1\}^{r}, \pm_{1} 1=-1}} \rho_{\Theta}(\epsilon) \exp \left(\pi i \sum_{\substack{i=2, \pm_{i} 1=\mp 1}}^{r} s_{i}\right)
$$

Now, since $\rho_{\Theta}(\epsilon)=\prod_{i \in \Theta}\left( \pm_{i} 1\right)=(-1)^{|\Theta \cap\{1\}|} \prod_{i \in \Theta \backslash\{1\}}\left( \pm_{i} 1\right)$, we have

$$
\begin{aligned}
A_{ \pm} & =(-1)^{|\Theta \cap\{1\}|}\left(\prod_{\substack{i=2, i \in \Theta}}^{r}\left( \pm 1 \mp e^{\pi i s_{i}}\right)\right)\left(\prod_{\substack{i=2, i \notin \Theta}}^{r}\left(1+e^{\pi i s_{i}}\right)\right) \\
& =( \pm 1)^{|\Theta \cap\{1\}|}(\mp 1)^{|\Theta|} i^{|\Theta \backslash\{1\}|} 2^{r-1} \exp \left(\frac{\pi i}{2} \sum_{i=2}^{r} s_{i}\right)\left(\prod_{\substack{i=2, i \in \Theta}}^{r} \sin \left(\frac{\pi s_{i}}{2}\right)\right)\left(\prod_{\substack{i=2, i \notin \Theta}}^{r} \cos \left(\frac{\pi s_{i}}{2}\right)\right)
\end{aligned}
$$

and thus

$$
\begin{aligned}
S & =\frac{2^{r-1}}{\pi}\left(\prod_{\substack{i \in \Theta, i \neq 1}} \sin \left(\frac{\pi s_{i}}{2}\right)\right)\left(\prod_{\substack{i \notin \Theta, i \neq 1}} \cos \left(\frac{\pi s_{i}}{2}\right)\right)(-1)^{|\Theta|} \Im\left(\left(e^{\pi i s_{1}}+(-1)^{|\Theta \cap\{1\}|}\right) e^{\frac{\pi i}{2}|\Theta \backslash\{1\}|+\frac{\pi i}{2} \sum_{i=2}^{r} s_{i}}\right) \\
& =\frac{2^{r}}{\pi}\left(\prod_{i \in \Theta} \sin \left(\frac{\pi s_{i}}{2}\right)\right)\left(\prod_{i \notin \Theta} \cos \left(\frac{\pi s_{i}}{2}\right)\right)(-1)^{|\Theta|} \Im\left(i^{|\Theta \cap\{1\}|} e^{\frac{\pi i}{2}|\Theta \backslash\{1\}|+\frac{\pi i}{2} \sum_{i=1}^{r} s_{i}}\right) \\
& =\frac{2^{r}}{\pi}\left(\prod_{i \in \Theta} \sin \left(\frac{\pi s_{i}}{2}\right)\right)\left(\prod_{i \notin \Theta} \cos \left(\frac{\pi s_{i}}{2}\right)\right) \sin \left(-\frac{\pi}{2}|\Theta|+\frac{\pi}{2} \sum_{i=1}^{r} s_{i}\right) .
\end{aligned}
$$

By the duplication and the reflection formula for the $\Gamma$-function we have

$$
\sin \left(\frac{\pi s}{2}\right) \Gamma(s)=\pi^{\frac{1}{2}} 2^{s-1} \frac{\Gamma\left(\frac{1}{2}+\frac{s}{2}\right)}{\Gamma\left(1-\frac{s}{2}\right)}, \quad \cos \left(\frac{\pi s}{2}\right) \Gamma(s)=\pi^{\frac{1}{2}} 2^{s-1} \frac{\Gamma\left(\frac{s}{2}\right)}{\Gamma\left(\frac{1}{2}-\frac{s}{2}\right)}
$$

and thus the Lemma follows.

Applying Lemma 19] with $r=\left|I_{1}\right|$ and using the definition (2.1) of $\Gamma_{i}$, we obtain

$$
\mathcal{X}_{I}\left(v_{1}, \boldsymbol{v}_{I}\right)=\Gamma\left(1-\sum_{i \in I_{1}} v_{i}\right) \frac{2^{\sum_{i \in I_{1}} v_{i}}}{\pi^{1-\frac{\left|I_{1}\right|}{2}}}\left(\prod_{i \in I_{1}} \frac{\Gamma_{i}\left(\frac{v_{i}}{2}\right)}{\Gamma_{i}\left(\frac{1}{2}-\frac{v_{i}}{2}\right)}\right) \sin \left(-\frac{\pi}{2}\left|I_{1} \cap \Upsilon\right|+\frac{\pi}{2} \sum_{i \in I_{1}} v_{i}\right) .
$$

Thus, plugging this expression into (7.30), making the change of variables $u_{i}=\frac{1}{2}-\alpha_{i}-v_{i}-s$ for all $i \in I$ and $u_{j} \rightarrow u_{j}+s$ for all $j \in(J \cup\{1\})$, and moving slightly the lines of integrations, we obtain

$$
\sum_{\substack{\epsilon \in\{ \pm 1\}^{k} \\ \pm_{1} 1=-1}} \rho_{\Upsilon}(\epsilon) \sum_{ \pm_{*} 1 \in\{ \pm 1\}} \mathcal{S}_{I ; \epsilon^{*} ; \boldsymbol{\alpha}, \boldsymbol{\beta}}=\mathcal{U}_{I_{1} ; \boldsymbol{\alpha}, \boldsymbol{\beta}}
$$


where for $\mathcal{I} \subseteq\{1, \ldots, k\}$ (with $|\mathcal{I}| \geq 2$ ), $\mathcal{J}:=\{1, \ldots, k\} \backslash \mathcal{I}$, we define

$$
\begin{aligned}
\mathcal{U}_{\mathcal{I} ; \boldsymbol{\alpha}, \boldsymbol{\beta}}:= & -2^{|\mathcal{J}|} i^{|\Upsilon \cap \mathcal{J}|} \sum_{\ell} \sum_{h(\bmod \ell)}^{*} \frac{P(\ell / L)}{(2 \pi i)^{1+k}} \int_{\left(c_{s}, \boldsymbol{c}_{\boldsymbol{u}}\right)} \frac{q^{k s-1}}{\pi \ell^{\sum_{i \in \mathcal{I}}\left(1-\alpha_{i}+\beta_{i}\right)}} \frac{H_{\mathcal{I} ; \boldsymbol{\alpha}, \boldsymbol{\beta}}^{\prime \prime}(s)}{s} \\
& \times\left(\prod_{j \in \mathcal{J}} D_{j ; \alpha_{j}, \beta_{j}}\left(\frac{1}{2}+u_{j}+s, \frac{h}{\ell}\right) \tilde{P}\left(u_{j}\right) N_{j}^{u_{j}}\right) \Gamma\left(1+|\mathcal{I}|\left(s-\frac{1}{2}\right)+\sum_{i \in \mathcal{I}}\left(u_{i}+\alpha_{i}\right)\right) \\
& \times \sin \left(\frac{\pi}{2}|\Upsilon \cap \mathcal{I}|+\frac{\pi}{2}|\mathcal{I}|\left(s-\frac{1}{2}\right)+\frac{\pi}{2} \sum_{i \in \mathcal{I}}\left(u_{i}+\alpha_{i}\right)\right) \\
& \times F\left(1+|\mathcal{I}|\left(s-\frac{1}{2}\right)+\sum_{i \in \mathcal{I}}\left(u_{i}+\alpha_{i}\right), \frac{q h}{\ell}\right)\left(\prod_{i \in \mathcal{I}} \frac{\pi^{\frac{1}{2}} \tilde{P}\left(u_{i}\right) N_{i}^{u_{i}}}{(2 q)^{u_{i}+\alpha_{i}+s-\frac{1}{2}}} \frac{\Gamma_{i}\left(\frac{1}{4}-\frac{u_{i}+\alpha_{i}+s}{2}\right)}{\Gamma_{i}\left(\frac{1}{4}+\frac{u_{i}+\alpha_{i}+s}{2}\right)}\right) d s \boldsymbol{d u},
\end{aligned}
$$

with lines of integration

$$
c_{u_{i}}=\frac{1}{2}-\Re\left(\alpha_{i}\right)-3 \frac{\varepsilon}{k}-\varepsilon \forall i \in\{1, \ldots, k\} \quad c_{s}:=\varepsilon / k,
$$

and, recalling (7.11),

$$
H_{\mathcal{I} ; \boldsymbol{\alpha}, \boldsymbol{\beta}}^{\prime \prime}(s):=G_{\boldsymbol{\alpha}, \boldsymbol{\beta}}(s) g_{\boldsymbol{\alpha}, \boldsymbol{\beta}}(s) \prod_{i \in \mathcal{I}} \zeta\left(1-\alpha_{i}+\beta_{i}\right)
$$

For future use we remark that if we move $c_{u_{i}^{\prime}}$ to $c_{u_{i}^{\prime}}=-\frac{1}{2}-\Re\left(\alpha_{1}\right)-5 \varepsilon$ for some $i^{\prime} \in \mathcal{I}$ we get

$$
\mathcal{U}_{\mathcal{I} ; \boldsymbol{\alpha}, \boldsymbol{\beta}} \ll N_{i^{\prime}}^{-1+A \varepsilon} q^{A \varepsilon}\left(N_{1} \cdots N_{k}\right)^{\frac{1}{2}} L^{-\varepsilon} .
$$

Also, if $|\mathcal{I}|>|\mathcal{J}|$, then moving the line of integration to $c_{u_{j}}$ to $c_{u_{j}}=-\frac{1}{2}+5 \frac{\varepsilon}{k}-\varepsilon$ for all $j \in \mathcal{J}$ (leaving the other lines of integration as in (17.33)), we obtain

$$
\mathcal{U}_{\mathcal{I} ; \boldsymbol{\alpha}, \boldsymbol{\beta}} \ll q^{-1+A \varepsilon}\left(N_{1} \cdots N_{k}\right)^{\frac{1}{2}} L^{-\varepsilon} \prod_{j \in \mathcal{J}} N_{j}^{-1+A \varepsilon} .
$$

Finally, moving $c_{s}$ to $c_{s}=\frac{1}{2}+B-3 \frac{\varepsilon}{k}$ and $c_{u_{i}}$ to $c_{u_{i}}=-B$ for all $i=1, \ldots, k$ we obtain

$$
\mathcal{U}_{\mathcal{I} ; \boldsymbol{\alpha}, \boldsymbol{\beta}} \ll_{B}\left(N_{1} \cdots N_{k} / q^{k}\right)^{-B} q^{\frac{k}{2}-1+A \varepsilon},
$$

if $|\mathcal{I}| \geq 2$. 
We are now ready to complete the proof of Lemma 10, Using (17.26), (17.29) and (7.31) we obtain

$$
\begin{aligned}
\sum_{\epsilon \in\{ \pm 1\}^{k}} \rho_{\Upsilon}(\epsilon) \mathcal{O}_{\epsilon, \alpha, \beta}^{\prime \prime}= & \sum_{L}^{\dagger} \sum_{\substack{I \cup J=\{2, \ldots, k\}, I \cap J=\emptyset,|I|>|J|}} \sum_{\substack{\left\{\alpha_{i}^{\prime}, \beta_{i}^{\prime}\right\}=\left\{\alpha_{i}, \beta_{i}\right\} \\
\left(\alpha_{j}^{\prime}, \beta_{j}^{\prime}\right)=\left(\alpha_{j}, \beta_{j}\right) \forall j \in J}} 2 \mathcal{U}_{I_{1} ; \boldsymbol{\alpha}, \boldsymbol{\beta}} \\
& +O\left(\frac{N_{1}^{A \varepsilon}}{q^{1-A \varepsilon}}\left(\frac{q^{\vartheta} N_{1}^{\frac{k}{2}}+q^{\frac{k}{2}-\frac{1}{3}+\frac{\vartheta}{3}}}{\left(N_{2} \cdots N_{k}\right)^{\frac{1}{2}}}+\left(q^{\frac{1}{6}+\frac{\vartheta}{3}} N_{1}^{-\frac{1}{2}}+1\right)\left(N_{2} \cdots N_{k}\right)^{\frac{1}{2}}\right)\right) \\
= & \mathcal{M}_{\alpha, \beta}+O\left(\frac{N_{1}^{A \varepsilon}}{q^{1-A \varepsilon}}\left(\frac{q^{\vartheta} N_{1}^{\frac{k}{2}}+q^{\frac{k}{2}-\frac{1}{3}+\frac{\vartheta}{3}}}{\left(N_{2} \cdots N_{k}\right)^{\frac{1}{2}}}+\left(q^{\frac{1}{6}+\frac{\vartheta}{3}} N_{1}^{-\frac{1}{2}}+1\right)\left(N_{2} \cdots N_{k}\right)^{\frac{1}{2}}\right)\right)
\end{aligned}
$$

where

$$
\mathcal{M}_{\alpha, \beta}\left(N_{1}, \cdots, N_{k}\right):=\sum_{L}^{\dagger} \sum_{\substack{\mathcal{I} \cup \mathcal{J}=\{1, \ldots, k\}, \mathcal{I} \cap \mathcal{J}=\emptyset,|\mathcal{I}|>|J|+1}} \sum_{\substack{\left\{\alpha_{i}^{\prime}, \beta_{i}^{\prime}\right\}=\left\{\alpha_{i}, \beta_{i}\right\} \\\left(\alpha_{j}^{\prime}, \beta_{j}^{\prime}\right)=\left(\alpha_{j}, \beta_{j}\right) \\ \forall j \in \mathcal{I},}} 2 \mathcal{U}_{\mathcal{I} ; \boldsymbol{\alpha}, \boldsymbol{\mathcal { J }}}
$$

and $\mathcal{U}_{\mathcal{I} ; \boldsymbol{\alpha}, \boldsymbol{\beta}}$ as defined in (7.32). Noticed that in the last step we used (7.36) to extend the sum over the subsets of $\{1 \ldots, k\}$ to include also the sets $\mathcal{I}$ that do not contain 1 . Moreover, by (7.35) and (7.36) we also have

$$
\mathcal{M}_{\alpha, \beta}\left(N_{1}, \cdots, N_{k}\right) \ll q^{A \varepsilon}\left(N_{1} \cdots N_{k}\right)^{\frac{1}{2}} N_{1}^{-1+A \varepsilon} .
$$

and thus the proof of Lemma 10 is complete. Also, by (7.37) for any $B>0$ we have

$$
\mathcal{M}_{\alpha, \beta}\left(N_{1}, \cdots, N_{k}\right) \ll_{B} q^{\frac{k}{2}-1+A \varepsilon}\left(N_{1} \cdots N_{k} / q^{k}\right)^{-B}
$$

We remark that we reached a formula for $\mathcal{M}_{\alpha, \beta}$ which is completely symmetric in the $N_{1}, \ldots, N_{k}$. This is important in order to remove the assumption that $N_{1}$ is the largest of $N_{1}, \ldots, N_{k}$, so that we can sum over the partitions of unity.

\section{Assembling the main terms}

In this section we prove Lemma 12 .

We start by moving $c_{u_{i}}$ to $c_{u_{i}}=0$ for all $i \in \mathcal{I}$ and $c_{s}$ to $\frac{1}{2}-3 \frac{\varepsilon}{k}$ (we can do this without passing through any pole nor having problem of convergence). Then, after extending the sum over the partitions of unity $L, N_{1}, \ldots, N_{k}$ using (7.39) and summing over them using Lemma 17 we obtain

$$
\sum_{\substack{N_{1}, \ldots, N_{k}, N_{1} \cdots N_{k} \ll q^{k+\varepsilon}}}^{\dagger} \mathcal{M}_{\alpha, \beta}\left(N_{1}, \cdots, N_{k}\right)=\sum_{\substack{\mathcal{I} \cup \mathcal{J}=\{1, \ldots, k\}, \mathcal{I} \cap \mathcal{J}=\emptyset,|\mathcal{I}|>|\mathcal{J}|+1}} \sum_{\substack{\left\{\alpha_{i}^{\prime}, \beta_{i}^{\prime}\right\}=\left\{\alpha_{i}, \beta_{i}\right\} \\\left(\alpha_{j}^{\prime}, \beta_{j}^{\prime}\right)=\left(\alpha_{j}, \beta_{j}\right) \\ \forall j \in \mathcal{J},}} 2 \mathscr{V}_{\mathcal{I} ; \boldsymbol{\alpha}, \boldsymbol{\beta}}+O(1)
$$


with

$$
\begin{aligned}
\mathscr{V}_{\mathcal{I} ; \boldsymbol{\alpha}, \boldsymbol{\beta}}:= & -2^{|\mathcal{J}|} i^{|\Upsilon \cap \mathcal{J}|} \frac{1}{2 \pi i} \int_{\left(c_{s}\right)} \sum_{\ell} \sum_{h}{ }_{(\bmod \ell)} \frac{q^{\frac{1}{2}|\mathcal{I}|+|\mathcal{J}| s-1}}{\ell^{\sum_{i \in \mathcal{I}}\left(1-\alpha_{i}+\beta_{i}\right)}} \Gamma\left(1+|\mathcal{I}|\left(s-\frac{1}{2}\right)+\sum_{i \in \mathcal{I}} \alpha_{i}\right) \\
& \times\left(\prod_{j \in \mathcal{J}} D_{j ; \alpha_{j}, \beta_{j}}\left(\frac{1}{2}+s, \frac{h}{\ell}\right)\right)\left(\prod_{i \in \mathcal{I}} \frac{(2 \pi)^{\frac{1}{2}}}{2^{\alpha_{i}+s} q^{\alpha_{i}}} \frac{\Gamma_{i}\left(\frac{1}{4}-\frac{\alpha_{i}+s}{2}\right)}{\Gamma_{i}\left(\frac{1}{4}+\frac{\alpha_{i}+s}{2}\right)}\right) \frac{H_{\mathcal{I} ; \boldsymbol{\alpha}, \boldsymbol{\beta}}^{\prime \prime}(s)}{\pi s} \\
& \times F\left(1+|\mathcal{I}|\left(s-\frac{1}{2}\right)+\sum_{i \in \mathcal{I}} \alpha_{i}, \frac{h}{\ell}\right) \sin \left(\frac{\pi}{2}|\Upsilon \cap \mathcal{I}|+\frac{\pi}{2}|\mathcal{I}|\left(s-\frac{1}{2}\right)+\frac{\pi}{2} \sum_{i \in \mathcal{I}} \alpha_{i}\right) d s,
\end{aligned}
$$

and line of integration $c_{s}=\frac{1}{2}-3 \frac{\varepsilon}{k}$.

Now, for each integral we move the line of integration $c_{s}$ to

$c_{s}=\max \left(0,-\frac{1}{2}+\frac{|\mathcal{J}|+\frac{3}{2}}{|\mathcal{I}|+|\mathcal{J}|-\frac{1}{2}}\right)+9 \frac{\varepsilon}{k}= \begin{cases}\frac{3}{4 k-2}+9 \frac{\varepsilon}{k} & \text { if }|\mathcal{I}|=|\mathcal{J}|+2=\frac{k}{2}+1 \text { with } k \text { even, } \\ 9 \frac{\varepsilon}{k} & \text { if }|\mathcal{I}|>|\mathcal{J}|+2 .\end{cases}$

picking up the residue of the pole of the $\Gamma$-function at

$$
s^{\prime}=s^{\prime}(\boldsymbol{\alpha})=\frac{1}{2}-\frac{1+\sum_{i \in \mathcal{I}} \alpha_{i}}{|\mathcal{I}|}
$$

(unless $k=4,|\mathcal{I}|=3$ in which case we stay on the right of such pole). Notice that Lemma 6 guarantees the convergence of the sum over $\ell$ on the new line of integration. Also, a quick computation shows that if $\mathcal{I} \neq I_{k}:=\{1, \ldots, k\}$ (and $|\mathcal{I}|>|\mathcal{J}|+1$ ) then

$$
\frac{1}{2}|\mathcal{I}|+|\mathcal{J}| c_{s}-1 \leq \frac{k}{2}-\frac{3}{2}+\iota_{k}+9 \frac{\varepsilon}{k}
$$

where $\iota_{k}=\frac{3}{14}$ if $k=4$ and $\iota_{k}=0$ otherwise. In particular, if $\mathcal{I} \neq I_{k}$, then by (3.9) the contribution of the integral on the new line of integration is $O\left(q^{\frac{k}{2}-\frac{3}{2}+\iota_{k}+A \varepsilon}\right)$ and we obtain

$$
\mathscr{V}_{\mathcal{I} ; \boldsymbol{\alpha}, \boldsymbol{\beta}}=\mathscr{X}_{\mathcal{I} ; \boldsymbol{\alpha}, \boldsymbol{\beta}}+O\left(q^{\frac{k}{2}-\frac{3}{2}+\iota_{k}+A \varepsilon}\right)
$$

where

$$
\begin{aligned}
\mathscr{X}_{\mathcal{I} ; \boldsymbol{\alpha}, \boldsymbol{\beta}}:= & -\frac{2^{|\mathcal{J}|} i^{|\Upsilon \cap \mathcal{J}|}}{|\mathcal{I}|} \sum_{\ell} \sum_{h(\bmod \ell)}^{*} \frac{q^{\frac{1}{2}|\mathcal{I}|+|\mathcal{J}| s^{\prime}-1}}{\ell^{\sum_{i \in \mathcal{I}}\left(1-\alpha_{i}+\beta_{i}\right)}} F\left(0, \frac{h}{\ell}\right) \sin \left(\frac{\pi}{2}(|\Upsilon \cap \mathcal{I}|-1)\right) \\
& \times\left(\prod_{j \in \mathcal{J}} D_{j ; \alpha_{j}, \beta_{j}}\left(\frac{1}{2}+s^{\prime}, \frac{h}{\ell}\right)\right)\left(\prod_{i \in \mathcal{I}} \frac{(2 \pi)^{\frac{1}{2}}}{2^{s^{\prime}+\alpha_{i}} q^{\alpha_{i}}} \frac{\Gamma_{i}\left(\frac{1}{4}-\frac{\alpha_{i}+s^{\prime}}{2}\right)}{\Gamma_{i}\left(\frac{1}{4}+\frac{\alpha_{i}+s^{\prime}}{2}\right)}\right) \frac{H_{\mathcal{I} ; \boldsymbol{\alpha}, \boldsymbol{\beta}}^{\prime \prime}\left(s^{\prime}\right)}{\pi s^{\prime}} .
\end{aligned}
$$

(Notice that (8.2) holds also in the case $k=4,|\mathcal{I}|=3$, since from (3.5) and a trivial bound it follows that $\mathscr{X}_{\mathcal{I} ; \boldsymbol{\alpha}, \boldsymbol{\beta}}$ is convergent and $\left.O\left(q^{\frac{2}{3}+A \varepsilon}\right)=O\left(q^{\frac{k}{2}-\frac{3}{2}+\iota_{k}+A \varepsilon}\right)\right)$. 
If $\mathcal{I}=I_{k}$, then

$$
\mathscr{V}_{I_{k} ; \boldsymbol{\alpha}, \boldsymbol{\beta}}=\mathscr{X}_{I_{k} ; \boldsymbol{\alpha}, \boldsymbol{\beta}}+\mathscr{V}_{I_{k} ; \boldsymbol{\alpha}, \boldsymbol{\beta}}^{\prime},
$$

where $\mathscr{V}_{I_{k} ; \boldsymbol{\alpha}, \boldsymbol{\beta}}^{\prime}$ is as in (8.1), but with the line of integration $c_{s}=9 \varepsilon / k$.

Now, notice that if $|\Upsilon \cap \mathcal{J}|$ is odd (and thus so is $|\Upsilon \cap \mathcal{I}|$ since $|\Upsilon|$ is even), then the sine in the expression defining $\mathscr{X}_{\mathcal{I} ; \boldsymbol{\alpha}, \boldsymbol{\beta}}$ is equal to 0 and thus so is $\mathscr{X}_{\mathcal{I} ; \boldsymbol{\alpha}, \boldsymbol{\beta}}$. If $|\Upsilon \cap \mathcal{J}|$ is even, then changing $h$ into $-h, \epsilon_{\mathcal{J}}$ into $-\epsilon_{\mathcal{J}}$ and using the identity $F(0, h / \ell)+F(0,-h / \ell)=-1$ (which follows immediately from (3.5)), we obtain $\mathscr{X}_{\mathcal{I} ; \boldsymbol{\alpha}, \boldsymbol{\beta}}=-\mathscr{X}_{\mathcal{I} ; \boldsymbol{\alpha}, \boldsymbol{\beta}}+\mathscr{K}_{\mathcal{I} ; \boldsymbol{\alpha}, \boldsymbol{\beta}}$ and so $\mathscr{X}_{\mathcal{I} ; \boldsymbol{\alpha}, \boldsymbol{\beta}}=$ $\frac{1}{2} \mathscr{K}_{\mathcal{I} ; \boldsymbol{\alpha}, \boldsymbol{\beta}}$, where

$$
\begin{aligned}
\mathscr{K}_{\mathcal{I} ; \boldsymbol{\alpha}, \boldsymbol{\beta}}:= & -\frac{2^{|\mathcal{J}|}}{|\mathcal{I}|} \sum_{\ell} \sum_{h(\bmod \ell)}^{*} \frac{q^{\frac{1}{2}|\mathcal{I}|+|\mathcal{J}| s^{\prime}-1}}{\ell^{\sum_{i \in \mathcal{I}}\left(1-\alpha_{i}+\beta_{i}\right)}}\left(\prod_{j \in \mathcal{J}} D_{j ; \alpha_{j}, \beta_{j}}\left(\frac{1}{2}+s^{\prime}, \frac{h}{\ell}\right)\right) \\
& \times\left(\prod_{i \in \mathcal{I}} \frac{(2 \pi)^{\frac{1}{2}}}{2^{s+\alpha_{i}} q^{\alpha_{i}}} \frac{\Gamma_{i}\left(\frac{1}{4}-\frac{\alpha_{i}+s^{\prime}}{2}\right)}{\Gamma_{i}\left(\frac{1}{4}+\frac{\alpha_{i}+s^{\prime}}{2}\right)}\right) \frac{H_{\mathcal{I} ; \boldsymbol{\alpha}, \boldsymbol{\beta}}^{\prime \prime}\left(s^{\prime}\right)}{\pi s^{\prime}} \\
= & -\mathscr{D}_{\mathcal{I} ; \boldsymbol{\alpha}, \boldsymbol{\beta} \boldsymbol{\beta}}^{\prime},
\end{aligned}
$$

where $\mathscr{D}_{\mathcal{I} ; \boldsymbol{\alpha}, \boldsymbol{\beta}}^{\prime}$ is as in (6.2), since $\frac{1}{2}|\mathcal{I}|+|\mathcal{J}| s^{\prime}-1=k s^{\prime}+\left(\frac{1}{2}-s^{\prime}\right)|\mathcal{I}|-1=k s^{\prime}+\sum_{i \in \mathcal{I}} \alpha_{i}$ and by the definition (7.34) of $H_{\mathcal{I} ; \boldsymbol{\alpha}, \boldsymbol{\beta}}^{\prime \prime}\left(s^{\prime}\right)$. It follows that

$$
\sum_{\substack{\mathcal{I} \cup \mathcal{J}=\{1, \ldots, k\}, \mathcal{I} \cap \mathcal{J}=\emptyset, \frac{k}{2}+\frac{3}{4}<|\mathcal{I}|}} \sum_{\substack{\left\{\alpha_{i}^{\prime}, \beta_{i}^{\prime}\right\}=\left\{\alpha_{i}, \beta_{i}\right\} \\\left(\alpha_{j}^{\prime}, \beta_{j}^{\prime}\right)=\left(\alpha_{j}, \beta_{j}\right)}} 2 \sum_{\forall j \in \mathcal{I}} \mathscr{\mathscr { V }}_{\mathcal{I} ; \boldsymbol{\alpha}, \boldsymbol{\beta}}=\sum_{\substack{\left\{\alpha_{i}^{\prime}, \beta_{i}^{\prime}\right\}=\left\{\alpha_{i}, \beta_{i}\right\} \\ \forall i \in I_{k}}} 2 \mathscr{V}_{I_{k} ; \boldsymbol{\alpha}, \boldsymbol{\beta}}^{\prime},-\mathscr{D}_{\boldsymbol{\alpha}, \boldsymbol{\beta}}+O\left(q^{\frac{k}{2}-\frac{1}{2}+\iota_{k}+A \varepsilon}\right)
$$

and thus to conclude the proof of Lemma 12, we just need to show $\mathscr{V}_{I_{k} ; \boldsymbol{\alpha}, \boldsymbol{\beta}}^{\prime}+X_{\boldsymbol{\beta}, \boldsymbol{\alpha}} \mathscr{V}_{I_{k} ;-\boldsymbol{\beta},-\boldsymbol{\alpha}}^{\prime}=$ $\mathscr{M}_{\boldsymbol{\alpha}, \boldsymbol{\beta}}$ with $\mathscr{M}_{\boldsymbol{\alpha}, \boldsymbol{\beta}}$ as in (2.3). First, we need the following lemma.

Lemma 20. For $\Re\left(s_{1}+s_{1}\right)>2$ and $\Re\left(s_{2}\right)>1$ we have

$$
\sum_{\ell} \frac{1}{\ell^{s_{2}}} \sum_{h(\bmod \ell)}^{*} F\left(s_{1}, \frac{h}{\ell}\right)=\frac{\zeta\left(s_{1}\right) \zeta\left(s_{1}+s_{2}-1\right)}{\zeta\left(s_{2}\right)} .
$$

Proof. From the functional equation for $F(s, x)$ and the Phragmén-Lindelöf principle one sees that if $\left|s_{1}-1\right|>\varepsilon^{\prime}>0$, then if

$$
\sum_{h(\bmod \ell)}^{*}\left|F\left(s_{1}, \frac{h}{\ell}\right)\right| \ll_{s, \varepsilon, \varepsilon^{\prime}} 1+\ell^{1-\Re\left(s_{1}\right)+\varepsilon}
$$


for all $\varepsilon>0$. It follows that the left hand side of (8.3) defines a meromorphic function in $s_{1}, s_{2}$ on the region $\Re\left(s_{2}\right)>1, \Re\left(s_{1}+s_{2}\right)>2$. Now, assume $\Re\left(s_{1}\right), \Re\left(s_{2}\right)>1$. Expanding $F$ into its Dirichlet expansion (3.3), executing the sum over $h$, and using (7.2), we obtain

$$
\sum_{\ell} \frac{1}{\ell^{s_{2}}} \sum_{h(\bmod \ell)}^{*} F\left(s_{1}, \frac{h}{\ell}\right)=\sum_{\ell} \frac{1}{\ell^{s_{2}}} \sum_{n} \frac{c_{\ell}(n)}{n^{s_{1}}}=\frac{1}{\zeta\left(s_{2}\right)} \sum_{n} \frac{\sigma_{1-s_{2}}(n)}{n^{s_{1}}}=\frac{\zeta\left(s_{1}\right) \zeta\left(s_{1}+s_{2}-1\right)}{\zeta\left(s_{2}\right)} .
$$

The Lemma then follows by analytic continuation.

Applying this Lemma, we see that

$$
\begin{aligned}
\mathscr{V}_{I_{k} ; \boldsymbol{\alpha}, \boldsymbol{\beta}}^{\prime}= & -\frac{q^{\frac{k}{2}-1}}{2 \pi i} \int_{\left(c_{s}\right)} \Gamma\left(1+k s-\frac{k}{2}+\sum_{i=1}^{k} \alpha_{i}\right) \frac{\zeta\left(1+k s-\frac{k}{2}+\sum_{i=1}^{k} \alpha_{i}\right) \zeta\left(\frac{k}{2}+k s+\sum_{i=1}^{k} \beta_{i}\right)}{\zeta\left(k-\sum_{i=1}^{k}\left(\alpha_{i}-\beta_{i}\right)\right)} \\
& \times \sin \left(\frac{\pi}{2}\left(|\Upsilon|-\frac{k}{2}+k s+\sum_{i=1}^{k} \alpha_{i}\right)\right)\left(\prod_{i=1}^{k} \frac{\Gamma_{i}\left(\frac{1}{4}-\frac{\alpha_{i}+s}{2}\right)}{\Gamma_{i}\left(\frac{1}{4}+\frac{\alpha_{i}+s}{2}\right)} \frac{\pi^{\frac{1}{2}} q^{-\alpha_{i}}}{2^{s-\frac{1}{2}+\alpha_{i}}}\right) \frac{H_{I_{k} ; \boldsymbol{\alpha}, \boldsymbol{\beta}}^{\prime \prime}(s)}{\pi s} d s,
\end{aligned}
$$

so that by the functional equation (using that $|\Upsilon|$ is even) and the definition (17.34) of $H^{\prime \prime}$ we obtain

$$
\begin{aligned}
& \mathscr{V}_{I_{k} ; \boldsymbol{\alpha}, \boldsymbol{\beta}}^{\prime}=(-1)^{\frac{|\Upsilon|}{2}} \frac{q^{\frac{k}{2}-1}}{2 \pi i} \int_{\left(c_{s}\right)} \frac{\zeta\left(\frac{k}{2}-k s-\sum_{i=1}^{k} \alpha_{i}\right) \zeta\left(\frac{k}{2}+k s+\sum_{i=1}^{k} \beta_{i}\right)}{\zeta\left(k-\sum_{i=1}^{k}\left(\alpha_{i}-\beta_{i}\right)\right)}
\end{aligned}
$$

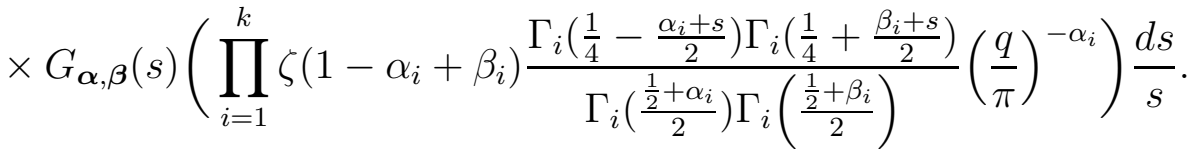

Notice that changing $s$ into $-s$ we obtain exactly $-X_{\boldsymbol{\beta}, \boldsymbol{\alpha}}$ times the analogous term coming from $\mathscr{V}_{I_{k} ;-\boldsymbol{\beta},-\boldsymbol{\alpha}}^{\prime}$, but with line of integration $c_{s}=-9 \frac{\varepsilon}{k}$. Thus, $\mathscr{V}_{I_{k} ; \boldsymbol{\alpha}, \boldsymbol{\beta}}^{\prime}+X_{\boldsymbol{\beta}, \boldsymbol{\alpha}} \mathscr{V}_{I_{k} ;-\boldsymbol{\beta},-\boldsymbol{\alpha}}^{\prime}$, coincides with the residue of the above integral at $s=0$, that is

$$
\begin{aligned}
\mathscr{V}_{I_{k} ; \boldsymbol{\alpha}, \boldsymbol{\beta}}^{\prime}+X_{\boldsymbol{\beta}, \boldsymbol{\alpha}} \mathscr{V}_{I_{k} ; \boldsymbol{\beta},-\boldsymbol{\alpha},}^{\prime}= & (-1)^{\frac{|\boldsymbol{|}|}{2}} q^{\frac{k}{2}-1} \frac{\zeta\left(\frac{k}{2}-\sum_{i=1}^{k} \alpha_{i}\right) \zeta\left(\frac{k}{2}+\sum_{i=1}^{k} \beta_{i}\right)}{\zeta\left(k-\sum_{i=1}^{k}\left(\alpha_{i}-\beta_{i}\right)\right)} \\
& \times \prod_{i=1}^{k} \zeta\left(1-\alpha_{i}+\beta_{i}\right) \frac{\Gamma_{i}\left(\frac{1}{4}-\frac{\alpha_{i}}{2}\right)}{\Gamma_{i}\left(\frac{1}{4}+\frac{\alpha_{i}}{2}\right)}\left(\frac{q}{\pi}\right)^{-\alpha_{i}} .
\end{aligned}
$$

Thus, Lemma 12 follows.

\section{The terms far from the diagonal}

We will use the following result of Young, to prove Lemma 11 
Lemma 21. Let $L, K \ll q^{1+\varepsilon}$ and let $W$ be a smooth function with compact support on $\mathbb{R}_{>0}$. Then,

$$
\sum_{0<\ell<L}\left|\sum_{(k, q)=1} \mathrm{e}\left(\frac{\ell \bar{k}}{q}\right) W\left(\frac{k}{K}\right)\right| \ll L^{\frac{1}{2}} q^{\frac{3}{4}+\varepsilon}+q^{\varepsilon} K^{\frac{1}{2}} L
$$

Proof. This is Proposition 4.3 of [You11b] (notice that we removed the condition $(q, \ell)=1$ from the first sum; one can easily check the bound holds also in this case).

Proof of Lemma 11. For simplicity we shall take $\boldsymbol{\alpha}=\boldsymbol{\beta}=\mathbf{0}:=(0, \ldots, 0)$, as the shifts don't play any role in this Lemma and the same argument with obvious modifications works also when $\alpha_{i}, \beta_{i} \ll 1 / \log q$.

By symmetry, we can assume that $N_{1}$ is the maximum of the $N_{i}$ and that $N_{2}$ is the second largest. Also, we assume $N_{1} \cdots N_{k} \ll q^{k+\varepsilon}$ and $N_{1} \gg q^{1+3 \varepsilon}$, since otherwise the result is trivial.

Now, we start by observing that we can remove the condition $\pm_{1} n_{1}+\cdots+ \pm_{k} n_{k} \neq 0$ in $\mathcal{O}_{\epsilon}^{\prime \prime}:=\mathcal{O}_{\epsilon, \mathbf{0}, \mathbf{0}}^{\prime \prime}$ at a cost of an admissible error:

$$
\begin{aligned}
\mathcal{O}_{\epsilon}^{\prime \prime}= & \sum_{d \mid q} d \frac{\mu\left(\frac{q}{d}\right)}{\varphi(q)} \sum_{d \mid\left( \pm_{1} n_{1} \pm_{2} \cdots \pm_{k} n_{k}\right)} \frac{d\left(n_{1}\right) \cdots d\left(n_{k}\right)}{\left(n_{1} \cdots n_{k}\right)^{\frac{1}{2}}} V_{\boldsymbol{\alpha}, \boldsymbol{\beta}}\left(\frac{n_{1} \cdots n_{k}}{q^{k}}\right) P\left(\frac{n_{1}}{N_{1}}\right) \cdots P\left(\frac{n_{k}}{N_{k}}\right) \\
& +O\left(q^{A \varepsilon}\left(N_{1} \cdots N_{k}\right)^{\frac{1}{2}} / N_{1}\right) .
\end{aligned}
$$

Next, we decompose $n_{1}$ into $n_{1}=f_{1} g_{1}$ and attach to the new variables two partitions of unity so that $f_{1} \asymp F_{1}, g_{1} \asymp G_{1}$ with $F_{1} \geq G_{1}$ and $F_{1} G_{1} \asymp N_{1}$. Writing $V$ and $P\left(\frac{n_{1}}{N_{1}}\right)$ in terms of their Mellin transform, we obtain

$$
\begin{aligned}
\mathcal{O}_{\epsilon}^{\prime \prime}= & \sum_{\substack{F_{1} G_{1} \asymp N_{1} \\
G_{1} \leq F_{1}}}^{\dagger} \int_{\left(c_{s}, c_{u_{1}}\right)}^{\prime} q^{k s} N_{1}^{u_{1}} \sum_{0 \leq|m|<M} w_{m}(s) \mathscr{A}_{m}\left(s+u_{1}\right) G_{\boldsymbol{\alpha}, \boldsymbol{\beta}}(s) g_{\boldsymbol{\alpha}, \boldsymbol{\beta}} \tilde{P}\left(u_{1}\right) \frac{d s}{s} d u_{1} \\
& +O\left(q^{A \varepsilon}\left(N_{1} \cdots N_{k}\right)^{\frac{1}{2}} / N_{1}\right),
\end{aligned}
$$

where $M:=\min \left(2 k N_{2}, q\right)$, the $\int^{\prime}$ indicates that the integrals are truncated at $\left|u_{1}\right|,|s| \leq q^{\varepsilon}$, the lines of integrations are $c_{u_{1}}=0, c_{s}=\varepsilon / k$, and

$$
\begin{aligned}
\mathscr{A}_{m}(s) & :=\sum_{d \mid q} d \frac{\mu\left(\frac{q}{d}\right)}{\varphi(q)} \sum_{g_{1}} \sum_{f_{1} g_{1} \equiv m(\bmod d)} \frac{1}{\left(f_{1} g_{1}\right)^{\frac{1}{2}+s}} P\left(\frac{f_{1}}{F_{1}}\right) P\left(\frac{g_{1}}{G_{1}}\right), \\
w_{m}(s):= & \sum_{ \pm_{2} n_{2} \pm_{3} \cdots \pm_{k} n_{k} \equiv-m(\bmod q)} \frac{d\left(n_{2}\right) \cdots d\left(n_{k}\right)}{\left(n_{2} \cdots n_{k}\right)^{\frac{1}{2}+s}} P\left(\frac{n_{2}}{N_{2}}\right) \cdots P\left(\frac{n_{k}}{N_{k}}\right) .
\end{aligned}
$$


Now, we apply Poisson's summation formula to the sum over $f_{1}$ and we see that for $\Re(s)=\varepsilon / k$

$$
\begin{aligned}
\mathscr{A}_{m}(s) & =\sum_{d \mid q} \frac{\mu\left(\frac{q}{d}\right)}{\varphi(q)} \sum_{g_{1}} \frac{P\left(g_{1} / G_{1}\right)}{g_{1}^{\frac{1}{2}+s}} \sum_{0 \leq|\ell| \leq \frac{d q}{\left(d, g_{1}\right) F_{1}}} \mathrm{e}\left(\frac{\ell m \overline{g_{1} /\left(d, g_{1}\right)}}{d /\left(d, g_{1}\right)}\right) \int_{0}^{\infty} \frac{P\left(x / F_{1}\right)}{x^{\frac{1}{2}+s}} \mathrm{e}\left(-\frac{\ell x}{d}\right) d x+O\left(q^{-1}\right) \\
& =\mathscr{A}_{m}^{*}(s)+O\left(q^{-1}\right),
\end{aligned}
$$

where

$$
\mathscr{A}_{m}^{*}(s)=\frac{F_{1}^{\frac{1}{2}-s}}{\varphi(q)} \sum_{\left(g_{1}, q\right)=1} \frac{P\left(g_{1} / G_{1}\right)}{g_{1}^{\frac{1}{2}+s}} \sum_{0<|\ell| \leq L} \mathrm{e}\left(\frac{\ell m \overline{g_{1}}}{q}\right) \int_{0}^{\infty} \frac{P(x)}{x^{\frac{1}{2}+s}} \mathrm{e}\left(-\frac{\ell F_{1} x}{q}\right) d x
$$

and $L=q^{1+A \varepsilon} / F_{1}$. Indeed, the sum over $\ell$ in the $d=1$ summands contains only the term $\ell=0$ which cancel with the $\ell=0$ term from $d=q$ (notice also that $\left(g_{1}, q\right)>1$ implies $\ell=0$ ). Thus, (9.1) becomes

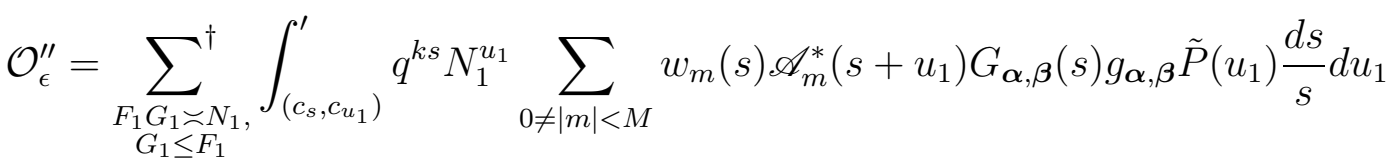

$$
\begin{aligned}
& +O\left(q^{\frac{k}{2}-\frac{3}{2}+A \varepsilon}+q^{A \varepsilon}\left(N_{1} \cdots N_{k}\right)^{\frac{1}{2}} / N_{1}\right),
\end{aligned}
$$

since the contribution of the terms with $m=0$ can be bounded trivially by

$$
\begin{aligned}
& \ll \frac{L\left(F_{1} G_{1}\right)^{\frac{1}{2}}}{q}\left(1+N_{2} / q\right) N_{2}^{-\frac{1}{2}}\left(N_{3} \cdots N_{k}\right)^{\frac{1}{2}} q^{A \varepsilon} \\
& \ll q^{A \varepsilon} N_{2}^{-\frac{1}{2}}\left(N_{3} \cdots N_{k}\right)^{\frac{1}{2}}+q^{-1+A \varepsilon}\left(N_{2} \cdots N_{k}\right)^{\frac{1}{2}} \ll q^{\frac{k}{2}-\frac{3}{2}+A \varepsilon}+q^{A \varepsilon}\left(N_{1} \cdots N_{k}\right)^{\frac{1}{2}} / N_{1} .
\end{aligned}
$$

since $N_{2}^{-1} N_{3} \cdots N_{k} \leq N_{4} \cdots N_{k} \ll q^{k-3+A \varepsilon}$. Also, we assume $N_{1} \leq F_{1}^{2} \leq q^{2+2 A \varepsilon}$, since otherwise $\mathscr{A}_{m}^{*}(s)$ is identically zero.

Changing the order of summation and integration and bounding trivially $G_{\boldsymbol{\alpha}, \boldsymbol{\beta}}(s) g_{\boldsymbol{\alpha}, \boldsymbol{\beta}} \tilde{P}\left(u_{1}\right)$, we see that

$$
\mathcal{O}_{\epsilon}^{\prime \prime} \ll \sum_{\substack{F_{1} G_{1} \asymp N_{1} \\ G_{1} \leq F_{1}}}^{\dagger} \int_{\substack{\left(c_{s}, c_{u_{1}}\right) \\ \prime}} q^{\varepsilon}\left|E\left(s, u_{1}\right)\right|\left|d s d u_{1}\right|+q^{\frac{k}{2}-\frac{3}{2}+A \varepsilon}+q^{A \varepsilon} \frac{\left(N_{1} \cdots N_{k}\right)^{\frac{1}{2}}}{N_{1}},
$$

where

$$
\begin{aligned}
E\left(s, u_{1}\right) & :=\frac{F_{1}^{\frac{1}{2}}}{\varphi(q)} \sum_{0<|\ell| \leq L} \sum_{0<|m|<M}\left|w_{m}(s)\right|\left|\sum_{\left(g_{1}, q\right)=1} \frac{P\left(g_{1} / G_{1}\right)}{g_{1}^{\frac{1}{2}+s+u_{1}}} \mathrm{e}\left(\frac{\ell m \overline{g_{1}}}{q}\right)\right| \\
& \ll \frac{F_{1}^{\frac{1}{2}}}{q G_{1}^{\frac{1}{2}}} \max _{0<|r| \leq R} c_{r} \sum_{0 \neq|r| \leq R}\left|\sum_{\left(g_{1}, q\right)=1} P\left(\frac{g_{1}}{G_{1}}\right)\left(\frac{G_{1}}{g_{1}}\right)^{\frac{1}{2}+s+u_{1}} \mathrm{e}\left(\frac{r \overline{g_{1}}}{q}\right)\right|,
\end{aligned}
$$


with $R:=\min \left(2 k L N_{2}, q\right) \leq 2 k q^{1+A \varepsilon} \min \left(N_{2} / F_{1}, 1\right)$ and

$$
\begin{aligned}
c_{r} & :=\sum_{\substack{\ell m \equiv r(\bmod q), 0<|m| \leq M, 0<|\ell| \leq L}}\left|w_{m}(s)\right| \ll \sum_{\substack{0<|\ell| \leq L, n_{2} \asymp N_{2}, \ldots, n_{k} \asymp N_{k},\left( \pm 2 n_{2} \pm 3 \cdots \pm_{k} n_{k}\right) \ell \equiv-r \\
(\bmod q)}} q^{A \varepsilon} d\left(n_{2}\right) \cdots d\left(n_{2}\right)\left(N_{2} \cdots N_{k}\right)^{-\frac{1}{2}} \\
& \ll \sum_{\substack{0<|\ell| \leq L,|n| \ll k N_{2}, n \ell \equiv-r(\bmod q)}} q^{A \varepsilon} N_{2}^{-\frac{1}{2}}\left(N_{3} \cdots N_{k}\right)^{\frac{1}{2}} \ll \sum_{\substack{|a| \ll k L N_{2}, a \equiv-r(\bmod q)}} d(a) q^{A \varepsilon} N_{2}^{-\frac{1}{2}}\left(N_{3} \cdots N_{k}\right)^{\frac{1}{2}} \\
& \ll q^{A \varepsilon} N_{2}^{-\frac{1}{2}}\left(N_{3} \cdots N_{k}\right)^{\frac{1}{2}}\left(1+L N_{2} / q\right) .
\end{aligned}
$$

Thus, by Lemma 21, for $|s|,\left|u_{1}\right| \ll q^{A \varepsilon}, \Re(s)=\varepsilon / k, \Re\left(u_{1}\right)=0$ we have

$$
\begin{aligned}
E\left(s, u_{1}\right) & \ll q^{-1+A \varepsilon} F_{1}^{\frac{1}{2}}\left(G_{1} N_{2}\right)^{-\frac{1}{2}}\left(N_{3} \cdots N_{k}\right)^{\frac{1}{2}}\left(1+L N_{2} / q\right)\left(R^{\frac{1}{2}} q^{\frac{3}{4}}+G_{1}^{\frac{1}{2}} R\right) \\
& \ll q^{A \varepsilon}\left(F_{1} / G_{1} N_{2}\right)^{\frac{1}{2}}\left(N_{3} \cdots N_{k}\right)^{\frac{1}{2}}\left(1+N_{2} / F_{1}\right) \min \left(F_{1}^{-\frac{1}{2}} N_{2}^{\frac{1}{2}} q^{\frac{1}{4}}+G_{1}^{\frac{1}{2}} N_{2} / F_{1}, q^{\frac{1}{4}}+G_{1}^{\frac{1}{2}}\right) \\
& =q^{A \varepsilon}\left(N_{3} \cdots N_{k}\right)^{\frac{1}{2}}\left(1+N_{2} / F_{1}\right) \min \left(\frac{q^{\frac{1}{4}}}{G_{1}^{\frac{1}{2}}}+\frac{N_{2}^{\frac{1}{2}}}{F_{1}^{\frac{1}{2}}} \frac{F_{1}^{\frac{1}{2}}}{N_{2}^{\frac{1}{2}}} \frac{q^{\frac{1}{4}}}{G_{1}^{\frac{1}{2}}}+\frac{F_{1}^{\frac{1}{2}}}{N_{2}^{\frac{1}{2}}}\right) .
\end{aligned}
$$

For $x, y>0$ we have $\left(1+x^{2}\right) \min (y+x, y / x+1 / x) \leq(x+1)(y+1)$, whence

$$
\begin{aligned}
E\left(s, u_{1}\right) & \ll q^{A \varepsilon}\left(N_{3} \cdots N_{k}\right)^{\frac{1}{2}}\left(N_{2}^{\frac{1}{2}} q^{\frac{1}{4}} N_{1}^{-\frac{1}{2}}+N_{2}^{\frac{1}{2}} F_{1}^{-\frac{1}{2}}+q^{\frac{1}{4}+\varepsilon} G_{1}^{-\frac{1}{2}}+1\right), \\
& \ll q^{A \varepsilon}\left(N_{3} \cdots N_{k}\right)^{\frac{1}{2}}\left(N_{2}^{\frac{1}{2}} N_{1}^{-\frac{1}{4}}+q^{\frac{3}{4}} N_{1}^{-\frac{1}{2}}+1\right), \\
& \ll q^{A \varepsilon}\left(\left(N_{2} \cdots N_{k}\right)^{\frac{1}{2}} N_{1}^{-\frac{1}{4}}+\left(N_{2} \cdots N_{k}\right)^{\frac{1}{2}-\frac{1}{2(k-1)}}\left(q^{\frac{3}{4}} N_{1}^{-\frac{1}{2}}+1\right)\right),
\end{aligned}
$$

where in the second inequality we used that $N_{1} \gg q, F_{1}^{-\frac{1}{2}} \leq N_{1}^{-\frac{1}{4}}, G_{1}^{-\frac{1}{2}} \asymp\left(F_{1} / N_{1}\right)^{\frac{1}{2}} \leq$ $N_{1}^{-\frac{1}{2}} q^{\frac{1}{2}+A \varepsilon}$ and $N_{1} \gg q$, and in the third one that $N_{3} \cdots N_{k} \leq N_{2}^{k-2}$ (so that $N_{3} \cdots N_{k} \leq$ $\left.\left(N_{2} \cdots N_{k}\right)^{\frac{k-2}{k-1}}\right)$. The lemma then follows by inserting (9.3) in (9.2).

\section{A Mellin formula}

In this section we prove a formula to separate the variables in expressions of the form $\left( \pm_{1} x_{1} \pm_{2}\right.$ $\left.\cdots \pm_{\kappa} x_{\kappa}\right)^{-s}$ which generalizes the Mellin transforms given in the following Lemma.

Lemma 22. Let $x, y>0$. Then

$$
(x+y)^{-b}=\frac{1}{2 \pi i} \int_{\left(c_{v}\right)} \frac{\Gamma(v) \Gamma(b-v)}{\Gamma(b)} x^{v-b} y^{-v} d v,
$$


for $0<c_{v}<\Re(b)$. Moreover, for $\Re(b)<0<c_{w}$, we have

$$
(x-y)^{-b} \chi_{\mathbb{R}_{>0}}(x-y)=\frac{1}{2 \pi i} \int_{\left(c_{w}\right)} \frac{\Gamma(w) \Gamma(1-b)}{\Gamma(1-b+w)} x^{w-b} y^{-w} d w,
$$

where $\chi_{X}(x)$ is the indicator function of the set $X$.

Equation (10.1) can be used repeatedly to give a formula for $\left(x_{1}+\cdots+x_{\kappa}\right)^{-s}$ valid for $\Re(s)>0$. However, it is not straightforward to obtain a satisfactory formula valid in the case when there are some minus signs, as the integrals obtained by repeatedly applying (10.1) and (10.2) are not absolutely convergent. The following Lemma overcomes this problem by introducing an extra integration.

Lemma 23. Let $\kappa \geq 2$ and $x_{1}, \ldots x_{\kappa}>0$. Let $\epsilon=\left( \pm_{1}, \cdots, \pm_{\kappa} 1\right) \in\{ \pm 1\}^{\kappa}$, with $\pm_{1} 1=-1$. Let $B \in \mathbb{Z}_{\geq 0}$ be such that $\frac{\kappa}{2}+\frac{1}{2}<\Re\left(v_{1}\right)<B+1$. Moreover, let $c_{v_{2}}, \ldots, c_{v_{\kappa}}, c_{v_{2}}^{\prime}, \ldots, c_{v_{\kappa}}^{\prime}>0$ be such that

$$
\Re\left(v_{1}\right)+c_{v_{2}}+\cdots+c_{v_{\kappa}}<B+1<\Re\left(v_{1}\right)+c_{v_{2}}^{\prime}+\cdots+c_{v_{\kappa}}^{\prime} .
$$

Then

$$
\begin{aligned}
& \left( \pm_{2} x_{2} \pm_{3} \cdots \pm_{\kappa} x_{\kappa}\right)^{v_{1}-1} \chi_{\mathbb{R}_{>} 0}\left( \pm_{2} x_{2} \pm_{3} \cdots \pm_{\kappa} x_{\kappa}\right) \\
& =\sum_{\substack{\nu=\left(\nu_{2}, \ldots, \nu_{\kappa}\right) \in \mathbb{Z}_{\geq 0}^{\kappa-1}, \nu_{2}+\cdots+\nu_{\kappa}=B, \nu_{i}=0 \text { if } \pm_{i}=-1}} \frac{B !}{\nu_{2} ! \cdots \nu_{\kappa} !} \frac{1}{(2 \pi i)^{\kappa-1}}\left(\int_{c_{\left.v_{2}, \ldots, c_{v_{\kappa}}\right)}}-\int_{\left(c_{v_{2}}^{\prime}, \ldots, c_{v_{\kappa}}^{\prime}\right)}\right) \frac{\Psi_{\epsilon, B}\left(v_{1}, \ldots, v_{\kappa}\right)}{x_{2}^{v_{2}-\nu_{2}} \cdots x_{\kappa}^{v_{\kappa}-\nu_{\kappa}}} d v_{2} \cdots d v_{\kappa} \\
&
\end{aligned}
$$

where

$$
\begin{aligned}
\Psi_{\epsilon, B}\left(s_{1}, \ldots, s_{\kappa}\right) & :=\frac{\Gamma\left(s_{1}\right) \cdots \Gamma\left(s_{\kappa}\right)}{\Gamma\left(V_{+; \epsilon}\left(s_{1}, \ldots, s_{\kappa}\right)\right) \Gamma\left(V_{-; \epsilon}\left(s_{1}, \ldots, s_{\kappa}\right)\right)} \frac{G\left(B+1-s_{1}-\cdots-s_{\kappa}\right)}{B+1-s_{1}-\cdots-s_{\kappa}}, \\
V_{ \pm ; \epsilon}\left(v_{1}, \ldots, v_{\kappa}\right) & :=\sum_{\substack{1 \leq i \leq \kappa, \pm_{i} 1= \pm 1}} v_{i}
\end{aligned}
$$

and $G(s)$ is any entire function such that $G(0)=1$ and $G(\sigma+i t) \ll e^{-C_{1}|t|}(1+|\sigma|)^{C_{2}|\sigma|}$ for some fixed $C_{1}, C_{2}>0$.

Remark 7. If $\epsilon=(-1, \ldots,-1)$, then $\Psi_{\epsilon}$ has to be interpreted as being identically zero.

Remark 8. If $\xi(s)$ is Riemann $\xi$-function, then $G(s)=\xi(s) / \xi(0)$ satisfies the hypothesis of the Lemma. 
Before giving a proof for Lemma 23, we give the following Lemma which implies that the integrals in (10.4) are absolutely convergent.

Lemma 24. Let $s_{i}=\sigma_{i}+i t_{i}$ for $i=1, \ldots, \kappa$. Then, for some $A>0$ we have

$$
\Psi_{\epsilon, B}\left(s_{1}, \ldots, s_{\kappa}\right) \ll \frac{1}{\delta^{\kappa}} \frac{\left(1+B+\left|\sigma_{1}\right|+\cdots+\left|\sigma_{\kappa}\right|\right)^{A\left(1+B+\left|\sigma_{1}\right|+\cdots+\left|\sigma_{\kappa}\right|\right)}}{\left(1+\left|t_{1}\right|\right)^{\frac{1}{2}-\sigma_{1}} \cdots\left(1+\left|t_{\kappa}\right|\right)^{\frac{1}{2}-\sigma_{\kappa}}\left(1+\left|t_{1}\right|+\cdots+\left|t_{\kappa}\right|\right)^{\sigma_{1}+\cdots+\sigma_{\kappa}-1}},
$$

provided that the $s_{i}$ are located at a distance greater than $\delta>0$ from the poles of $\Psi_{\epsilon}$.

Proof. By Stirling's formula (and the reflection's formula for the Gamma function), if the distance of $s=\sigma+i t$ from the poles of $\Gamma(s)$ is greater than $\delta$, then we have

$$
\begin{aligned}
\Gamma(s) & \ll \frac{1}{\delta}\left(1+A_{1}|\sigma|\right)^{|\sigma|}(1+|t|)^{\sigma-\frac{1}{2}} e^{-\frac{\pi}{2}|t|}, \\
\Gamma(s)^{-1} & \ll\left(1+A_{1}|\sigma|\right)^{|\sigma|}(1+|t|)^{-\sigma+\frac{1}{2}} e^{\frac{\pi}{2}|t|},
\end{aligned}
$$

for some $A_{1}>0$. It follows that

$$
\begin{aligned}
\Psi_{\epsilon, B}\left(s_{1}, \ldots, s_{\kappa}\right) \ll & \frac{1}{\delta^{\kappa}} \frac{\left(1+B+\left|\sigma_{1}\right|+\cdots+\left|\sigma_{\kappa}\right|\right)^{A_{2}\left(1+B+\left|\sigma_{1}\right|+\cdots+\left|\sigma_{\kappa}\right|\right)}}{\left(1+\left|t_{1}\right|\right)^{\frac{1}{2}-\sigma_{1}} \cdots\left(1+\left|t_{\kappa}\right|\right)^{\frac{1}{2}-\sigma_{\kappa}}} \times \\
& \times \frac{e^{-\frac{\pi}{2}\left(\left|t_{1}\right|+\cdots+\left|t_{\kappa}\right|-\left|V_{+; \epsilon}\left(t_{1}, \ldots, t_{\kappa}\right)\right|-\left|V_{-; \epsilon}\left(t_{1}, \ldots, t_{\kappa}\right)\right|\right)-C_{1}\left|t_{1}+\cdots+t_{\kappa}\right|}}{\left(1+\left|V_{+; \epsilon}\left(t_{1}, \ldots, t_{\kappa}\right)\right|\right)^{V_{+; \epsilon}\left(\sigma_{1}, \ldots, \sigma_{\kappa}\right)-\frac{1}{2}}\left(1+\left|V_{-; \epsilon}\left(t_{1}, \ldots, t_{\kappa}\right)\right|\right)^{V_{-; \epsilon}\left(\sigma_{1}, \ldots, \sigma_{\kappa}\right)-\frac{1}{2}}},
\end{aligned}
$$

for some $A_{2}>0$. Now, we have

$$
\frac{e^{-C_{1}|x+y|}}{(1+|x|)^{\eta_{1}}(1+|y|)^{\eta_{2}}} \ll \frac{\left(1+\left|\eta_{1}\right|+\left|\eta_{2}\right|\right)^{A_{3}\left(\left|\eta_{1}\right|+\left|\eta_{2}\right|\right)}}{(1+|x|+|y|)^{\eta_{1}+\eta_{2}}}
$$

for some $A_{3}>0$ (depending on $C_{1}$ ). Thus,

$$
\begin{aligned}
& \frac{e^{-\frac{\pi}{2}\left(\left|t_{1}\right|+\cdots+\left|t_{\kappa}\right|-\left|V_{+; \epsilon}\left(t_{1}, \ldots, t_{\kappa}\right)\right|-\left|V_{-; \epsilon}\left(t_{1}, \ldots, t_{\kappa}\right)\right|\right)-C_{1}\left|t_{1}+\cdots+t_{\kappa}\right|}}{\left(1+\left|V_{+; \epsilon}\left(t_{1}, \ldots, t_{\kappa}\right)\right|\right)^{V_{+; \epsilon}\left(\sigma_{1}, \ldots, \sigma_{\kappa}\right)-\frac{1}{2}}\left(1+\left|V_{-; \epsilon}\left(t_{1}, \ldots, t_{\kappa}\right)\right|\right)^{V_{-; \epsilon}\left(\sigma_{1}, \ldots, \sigma_{\kappa}\right)-\frac{1}{2}}} \\
& \quad \ll\left(1+\left|\sigma_{1}\right|+\cdots+\left|\sigma_{\kappa}\right|\right)^{A_{4}\left(\left|\sigma_{1}\right|+\cdots+\left|\sigma_{\kappa}\right|\right)} \frac{e^{-\frac{\pi}{2}\left(\left|t_{1}\right|+\cdots+\left|t_{\kappa}\right|-\left|V_{+; \epsilon}\left(t_{1}, \ldots, t_{\kappa}\right)\right|-\left|V_{-; \epsilon}\left(t_{1}, \ldots, t_{\kappa}\right)\right|\right)}}{\left(1+\left|V_{+; \epsilon}\left(t_{1}, \ldots, t_{\kappa}\right)\right|+\left|V_{-; \epsilon}\left(t_{1}, \ldots, t_{\kappa}\right)\right|\right)^{\sigma_{1}+\cdots+\sigma_{\kappa}-1}} \\
& \quad \ll \frac{\left(1+\left|\sigma_{1}\right|+\cdots+\left|\sigma_{\kappa}\right|\right)^{A_{5}\left(\left|\sigma_{1}\right|+\cdots+\left|\sigma_{\kappa}\right|\right)}}{\left(1+\left|t_{1}\right|+\cdots+\left|t_{\kappa}\right|\right)^{\sigma_{1}+\cdots+\sigma_{\kappa}-1}},
\end{aligned}
$$

and (10.6) follows. 
Proof of Lemma 23. First, we remark that the estimate (10.6) implies the absolute convergence of the integrals on the right hand side of (10.4) and justifies the following computations.

We prove the Lemma by induction. First we consider the case $\kappa=2$. From (10.1) we have

$$
\begin{aligned}
\left(x_{2}+x_{3}\right)^{v_{1}-1} & =\left(x_{2}+x_{3}\right)^{B}\left(x_{2}+x_{3}\right)^{v_{1}-1-B} \\
& =\sum_{\substack{\nu_{2}, \nu_{3} \in \mathbb{Z}_{\geq 0}, \nu_{2}+\nu_{3}=B}} \frac{B !}{\nu_{2} ! \nu_{3} !} x^{\nu_{2}} x_{3}^{\nu_{3}} \frac{1}{2 \pi i} \int_{\left(c_{v_{3}}\right)} \frac{\Gamma\left(v_{3}\right) \Gamma\left(1+B-v_{1}-v_{3}\right)}{\Gamma\left(1+B-v_{1}\right) x_{2}^{B+1-v_{1}-v_{3}} x_{3}^{v_{3}}} d v_{3},
\end{aligned}
$$

for $0<c_{v_{3}}<1+B-\Re\left(v_{1}\right)$. Now, by contour integration,

$\frac{\Gamma\left(1+B-v_{1}-v_{3}\right)}{\Gamma\left(1+B-v_{1}\right)} x_{2}^{v_{1}+v_{3}-B-1}=\frac{1}{2 \pi i}\left(\int_{\left(c_{v_{2}}\right)}-\int_{\left(c_{v_{2}}^{\prime}\right)}\right) \frac{\Gamma\left(v_{2}\right) x_{2}^{-v_{2}}}{\Gamma\left(v_{2}+v_{3}\right)} \frac{G\left(B+1-v_{1}-v_{2}-v_{3}\right)}{B+1-v_{1}-v_{2}-v_{3}} d v_{2}$,

where $c_{v_{2}}, c_{v_{2}}^{\prime}>0$ and $c_{v_{2}}<-\Re\left(v_{1}+v_{3}\right)+B+1<c_{v_{2}}^{\prime}$. Inserting this into (10.7) we obtain (10.4) in the case $\epsilon=(-1,1,1)$.

The case $\epsilon=(-1,1,-1)$ (and thus its permutation $\epsilon=(-1,-1,1))$ follows in the same way from (10.2).

Now, let $\epsilon=\left(-1, \pm_{2}, \ldots, \pm_{\kappa+1}\right) \in\{ \pm 1\}^{\kappa+1}$ with $\kappa \geq 2$ and suppose (10.4) holds for all $\epsilon^{\prime} \in\{ \pm 1\}^{\kappa}$ with $\pm_{1}^{\prime} 1=-1$. Since $\kappa+1 \geq 3$ there are two indexes $2 \leq i<j \leq \kappa+1$ such that $\pm_{i} 1= \pm_{j} 1$ and without loss of generality we can assume $i=\kappa, j=\kappa+1$. Then, letting $\epsilon^{\prime}=\left(-1, \pm_{2}, \ldots, \pm_{\kappa}\right)$, we have

$$
\begin{gathered}
\left.\left( \pm_{2} x_{2} \pm_{3} \cdots \pm_{\kappa+1} x_{\kappa+1}\right)\right)^{v_{1}-1} \chi_{\mathbb{R}_{>0}}\left( \pm_{2} x_{2} \pm_{3} \cdots \pm_{\kappa+1} x_{\kappa+1}\right)=\sum_{\substack{\nu=\left(\nu_{2}, \ldots, \nu_{\kappa}\right) \in \mathbb{Z}_{20}^{\kappa}, \nu_{2}+\cdots+\nu_{\kappa+1}=\bar{B}, \nu_{i}=0 \text { if } \pm_{i}=-1}} \frac{B !}{\nu_{2} ! \cdots \nu_{\kappa} !} \frac{1}{(2 \pi i)^{\kappa-1}} \times \\
\times\left(\int_{\left(c_{\left.v_{2}, \ldots, c_{v_{\kappa}}\right)}\right.}-\int_{\left(c_{v_{2}}^{\prime}, \ldots, c_{\nu_{\kappa}}^{\prime}\right)}\right) \frac{\Psi_{\epsilon^{\prime}, B}\left(v_{1}, \ldots, v_{\kappa}\right)}{x_{2}^{v_{2}-\nu_{2}} \cdots x_{\kappa-1}^{v_{\kappa}-\nu_{\kappa-1}}}\left(x_{\kappa}+x_{\kappa+1}\right)^{-v_{\kappa}+\nu_{\kappa}} d v_{2} \cdots d v_{\kappa}
\end{gathered}
$$

where $c_{v_{2}}, \ldots, c_{v_{\kappa}}, c_{v_{2}}^{\prime}, \ldots, c_{v_{\kappa}}^{\prime}>0$ satisfy (10.3). Then, we expand the binomial $\left(x_{\kappa}+x_{\kappa+1}\right)^{\nu_{\kappa}}$ and apply (10.1) to $\left(x_{\kappa}+x_{\kappa+1}\right)^{-v_{\kappa}}$. We obtain

$$
\begin{aligned}
\left( \pm_{2} x_{2} \pm_{3} \cdots\right. & \left.\left. \pm_{\kappa+1} x_{\kappa+1}\right)\right)^{v_{1}-1} \chi_{\mathbb{R}_{>0}}\left( \pm_{2} x_{2} \pm_{3} \cdots \pm_{\kappa+1} x_{\kappa+1}\right)= \\
= & \sum_{\substack{\nu=\left(\nu_{2}, \ldots, \nu_{\left.\nu_{+}\right)}\right) \in \mathbb{Z}_{20}^{\kappa}, \nu_{2}+\cdots+\nu_{\kappa}+1=B, \nu_{i}=0 \text { if } \pm_{i}=-1}} \frac{B !}{\nu_{2} ! \cdots \nu_{\kappa+1} !} \frac{1}{(2 \pi i)^{\kappa}}\left(\int_{\left(c_{v_{2}}, \ldots, c_{v_{\kappa+1}}\right)}-\int_{\left(c_{v_{2}}^{\prime}, \ldots, c_{v_{\kappa+1}}^{\prime}\right)}\right) \times \\
& \times \frac{\Psi_{\epsilon^{\prime}, B}\left(v_{1}, \ldots, v_{\kappa}\right)}{x_{2}^{v_{2}-\nu_{2}} \cdots x_{\kappa-1}^{v_{\kappa}-\nu_{\kappa-1}} x_{\kappa}^{v_{\kappa}-v_{\kappa+1}-\nu_{\kappa}} x_{\kappa+1}^{v_{\kappa+1}-\nu_{\kappa+1}}} \frac{\Gamma\left(v_{\kappa+1}\right) \Gamma\left(v_{\kappa}-v_{\kappa+1}\right)}{\Gamma\left(v_{\kappa}\right)} d v_{2} \cdots d v_{\kappa},
\end{aligned}
$$

where $c_{v_{\kappa+1}}, c_{v_{\kappa+1}}^{\prime}>0$ are such that $0<c_{v_{\kappa+1}}<c_{v_{\kappa}}$. We make the change of variables $v_{\kappa} \rightarrow v_{\kappa}+v_{\kappa+1}$ and the lemma follows. 
Lemma 25. Let $\kappa \geq 2$ and let $\epsilon^{*}=\left( \pm_{1} 1, \cdots, \pm_{\kappa} 1, \pm_{*} 1\right) \in\{ \pm 1\}^{\kappa+1}$, with $\pm_{1} 1=-1$. For $B \geq 0$, let

$$
\Psi_{\epsilon^{*}, B}^{\prime}\left(v_{1}, \ldots, v_{\kappa}\right):=\frac{\Gamma\left(B+1-v_{1}-\cdots-v_{\kappa}\right) \Gamma\left(v_{1}\right) \cdots \Gamma\left(v_{\kappa}\right)}{\Gamma\left(V_{\mp * ; \epsilon}\left(v_{1}, \ldots, v_{\kappa}\right)\right) \Gamma\left(B+1-V_{\mp * ;}\left(v_{1}, \ldots, v_{\kappa}\right)\right)}
$$

where $V_{ \pm ; \epsilon}$ is defined in (10.5).

Let $\mathcal{F}\left(v_{0}, \ldots, v_{\kappa}\right)$ be analytic on $\left\{\left(v_{0}, \ldots, v_{\kappa}\right) \in \mathbb{C}^{\kappa+1} \mid 0<\Re\left(v_{0}\right)<B+1\right\}$ and assume that for $0<\Re\left(v_{0}\right)<B+1$ and any $A>0$ one has that $\mathcal{F}$ satisfies

$$
\mathcal{F}\left(v_{0}, \ldots, v_{\kappa}\right) \ll \prod_{i=2}^{\kappa}\left(1+\left|v_{i}\right|\right)^{-A}
$$

where the implicit constant may depend on $A, v_{1}$ and $\Re\left(v_{0}\right)$. Then for any $v_{1} \in \mathbb{C}$ and $c_{v_{2}}, \ldots, c_{v_{\kappa}}>0$ satisfying $0<\Re\left(v_{1}\right)+c_{v_{2}}+\cdots+c_{v_{\kappa}}<1$ we have

$$
\begin{aligned}
& \sum_{\nu} \frac{B !}{\nu_{*} ! \nu_{2} ! \cdots \nu_{\kappa} !} \int_{\left(c_{v_{2}}, \ldots, c_{v_{\kappa}}\right)} \Psi_{\epsilon, B}^{\prime}\left(v_{1}, \ldots, v_{\kappa}\right) \times \\
& \quad \times \mathcal{F}\left(B+1-\nu_{*}-v_{1}-\cdots-v_{\kappa}, v_{1}, v_{2}-\nu_{2} \ldots, v_{\kappa}-\nu_{\kappa}\right) d v_{2} \cdots d v_{\kappa} \\
& =\int_{\left(c_{v_{2}}, \ldots, c_{v_{\kappa}}\right)} \Psi_{\epsilon, 0}^{\prime}\left(v_{1}, \ldots, v_{\kappa}\right) \mathcal{F}\left(1-v_{1}-\cdots-v_{\kappa}, v_{1}, \ldots, v_{\kappa}\right) d v_{2} \cdots d v_{\kappa}
\end{aligned}
$$

where the sum on the left is taken over $\nu=\left(\nu_{2}, \ldots, \nu_{\kappa}, \nu_{*}\right) \in \mathbb{Z}_{\geq 0}^{\kappa}$ satisfying

$$
\nu_{2}+\cdots+\nu_{\kappa}+\nu_{*}=B, \quad \nu_{i}=0 \text { if } \pm_{i}=-1 \text { or } i \in J, \quad \nu_{*}=0 \text { if } \pm_{*}=-1 .
$$

Proof. Making the change of variables $v_{i} \rightarrow v_{i}+\nu_{i}$, for $i=2, \ldots, \kappa$, moving back the lines of integration to $c_{v_{i}}$ (as we can do without crossing any pole), and switching the order of summation and integration, we see that the left hand side of (10.8) is equal to

$$
\begin{aligned}
\int_{\left(c_{v_{2}}, \ldots, c_{v_{\kappa}}\right)} \sum_{\nu} \frac{B !}{\nu_{*} ! \nu_{2} ! \cdots \nu_{\kappa} !} \Psi_{\epsilon}^{\prime}\left(v_{1}, v_{2}+\nu_{2}, \ldots, v_{\kappa}+\nu_{\kappa}\right) \\
\quad \times \mathcal{F}\left(1-v_{1}-\cdots-v_{\kappa}, v_{1}, \ldots, v_{\kappa}\right) d v_{2} \cdots d v_{\kappa} .
\end{aligned}
$$

Now, the identity $\mathrm{B}\left(s_{1}+1, s_{2}\right)+\mathrm{B}\left(s_{1}, s_{2}+1\right)=\mathrm{B}\left(s_{1}, s_{2}\right)$, satisfied by the Beta function $\mathrm{B}\left(s_{1}, s_{2}\right):=\Gamma\left(s_{1}\right) \Gamma\left(s_{2}\right) \Gamma\left(s_{1}+s_{2}\right)^{-1}$, can be generalized to

$$
\sum_{\substack{\left(r_{1}, \ldots, r_{m}\right) \in \mathbb{Z}_{\geq 0}^{m}, r_{1}+\cdots+r_{m}=r}} \frac{r !}{r_{1} ! \cdots r_{m} !} \frac{\Gamma\left(s_{1}+r_{1}\right) \cdots \Gamma\left(s_{m}+r_{m}\right)}{\Gamma\left(r+s_{1}+\cdots+s_{m}\right)}=\frac{\Gamma\left(s_{1}\right) \cdots \Gamma\left(s_{m}\right)}{\Gamma\left(s_{1}+\cdots+s_{m}\right)},
$$


for $m, r \geq 1, s_{1}, \ldots, s_{m} \in \mathbb{C}$. Thus, we have

$$
\sum_{\substack{\nu=\left(\nu_{2}, \ldots, \nu_{\kappa}, \nu_{*}\right) \in \mathbb{Z}_{20}^{\kappa}, \nu_{2}+\cdots+\nu_{\kappa}+\nu_{*}=B, \nu_{i}=0 \text { if } \pm_{i}=-1, \nu_{*}=0 \text { if } \pm_{*}=-1}} \frac{B !}{\nu_{*} ! \nu_{2} ! \cdots \nu_{\kappa} !} \Psi_{\epsilon, B}\left(v_{1}, v_{2}+\nu_{2} \ldots, v_{\kappa}+\nu_{\kappa}\right)=\Psi_{\epsilon, 0}^{\prime}\left(v_{1}, \ldots, v_{\kappa}\right)
$$

and the Lemma follows.

\section{References}

[Apo] Apostol, T.M. Introduction to analytic number theory. Undergraduate Texts in Mathematics. Springer-Verlag, New York-Heidelberg, 1976. xii+338 pp.

[BV] Baladi, V.; Vallée, B. Euclidean algorithms are Gaussian. J. Number Theory 110 (2005), no. 2, 331-386.

[Bec] Beck, M. Dedekind cotangent sums. Acta Arith. 109 (2003), no. 2, 109-130.

[Bet15] Bettin, S. On the distribution of a cotangent sum. Internat. Math. Res. Research Notices (2015) no. 21, 11419-11432.

[Bet16] Bettin, S.; On the reciprocity law for the twisted second moment of Dirichlet Lfunctions. Trans. Amer. Math. Soc. 368 (2016), 6887-6914.

[Bet] Bettin, S.; Linear correlations of the divisor function, preprint.

[BC13a] Bettin, S.; Conrey, J.B. A reciprocity formula for a cotangent sum. Internat. Math. Res. Research Notices, 2013 no. 24, 5709-5726.

[BC13b] Bettin, S.; Conrey, J.B. Period functions and cotangent sums. Algebra Number Theory 7 (2013), no. 1, 215-242.

[BFKMM] Blomer, V.; Fouvry, É.; Kowalski, E.; Michel, P.; Milićević, D. On moments of twisted L-functions, to appear in American Journal of Math.

[BHM] Blomer, V.; Harcos, G.; Michel, P. A Burgess-like subconvex bound for twisted Lfunctions. Appendix 2 by Z. Mao. Forum Math. 19 (2007), no. 1, 61-105.

[BM] Blomer, V.; Milićević, D. The second moment of twisted modular L-functions, Geom. Funct. Anal. (2015) 25, no. 2, 453-516. 
[Byk] Bykovskii, V.A. An estimate for the dispersion of lengths of finite continued fractions. Fundam. Prikl. Mat. 11 (2005), no. 6, 15-26; translation in J. Math. Sci. (N. Y.) 146 (2007), no. 2, 5634-5643

[Chi] Chinta, G. Mean values of biquadratic zeta functions. Invent. Math. 160 (2005), no. 1, $145-163$.

[CG] Conrey, J.B.; Ghosh, A. Remarks on the generalized Lindelöf hypothesis. Funct. Approx. Comment. Math. 36 (2006), 71-78.

[CGG] Conrey, J.B.; Ghosh, A.; Gonek, S.M. Large gaps between zeros of the zeta-function. Mathematika 33 (1986), no. 2, 212-238 (1987).

[CI] Conrey, J.B.; Iwaniec, H. The cubic moment of central values of automorphic L-functions. Ann. of Math. (2) 151 (2000), no. 3, 1175-1216.

[DI] Deshouillers, J.-M.; Iwaniec, H. Kloosterman sums and Fourier coefficients of cusp forms. Invent. Math. 70 (1982/83), no. 2, 219-288.

[Est30] Estermann, T. On the representation of a number as the sum of two products. Proc. London Math. Soc. (2), 31 (1930), 123-133.

[Est32] Estermann, T. On the representations of a number as the sum of three or more products. Proc. London Math. Soc. (2), 34 (1932), 190-195.

[FGKM] Fouvry, É; Ganguly, S.; Kowalski, E.; Michel, P. Gaussian distribution for the divisor function and Hecke eigenvalues in arithmetic progressions. Comment. Math. Helv. 89 (2014), no. 4, 979-1014.

[HL] Hardy G.H; Littlewood, J.E. Contributions to the theory of the Riemann zeta-function and the theory of the distribution of primes. Acta Mathematica 41 (1918), 119-196.

[HWW] Harman, G.; Watt, N.; Wong, K. A new mean-value result for Dirichlet L-functions and polynomials. Q. J. Math. 55 (2004), no. 3, 307-324.

[H-B79] Heath-Brown, D.R. The fourth power moment of the Riemann zeta function. Proc. London Math. Soc. (3) 38 (1979), no. 3, 385-422

[H-B81] Heath-Brown, D.R. The fourth power mean of Dirichlet's L-functions. Analysis 1 (1981), no. 1, 25-32.

[Hei] Heilbronn, H. On the average length of a class of finite continued fractions. 1969 Number Theory and Analysis (Papers in Honor of Edmund Landau) pp. 87-96 Plenum, New York 
[Hen] Hensley, D. The number of steps in the Euclidean algorithm. J. Number Theory 49 (1994), no. 2, 142-182.

[Ish] Ishibashi, M. The value of the Estermann zeta functions at $s=0$. Acta Arith. 73 (1995), no. $4,357-361$.

[IS] Iwaniec, H.; Sarnak, P. The non-vanishing of central values of automorphic L-functions and Landau-Siegel zeros. Israel J. Math. 120 (2000), part A, 155-177.

[Kim] Kim, H.H. Functoriality for the exterior square of $\mathrm{GL}_{4}$ and the symmetric fourth of $\mathrm{GL}_{2}$. With appendix 1 by Dinakar Ramakrishnan and appendix 2 by Kim and Peter Sarnak. J. Amer. Math. Soc. 16 (2003), no. 1, 139-183.

[LY] Lester, S.; Yesha, N. On the distribution of the divisor function and Hecke eigenvalues. Isr. J. Math. 212 (2016), no. 1, 443-472

[MR] Maier, H.; Rassias, M.T.; Generalizations of a cotangent sum associated to the Estermann zeta function. Commun. Contemp. Math. 18 (2016), no. 1, 1550078, 89 pp.

[Mot80] Motohashi, Y. An asymptotic series for an additive divisor problem. Math. Z. 170 (1980), no. 1, 43-63.

[Mot94] Motohashi, Y. The binary additive divisor problem. Ann. Sci. École Norm. Sup. (4) 27 (1994), no. 5, 529-572.

[Mot97] Motohashi, Y. Spectral Theory of the Riemann Zeta-Function. Cambridge Tracts in Math. 127, Cambridge Univ. Press, Cambridge, 1997.

[Pal] Paley, R.E.A.C. On the k-Analogues of some Theorems in the Theory of the Riemann

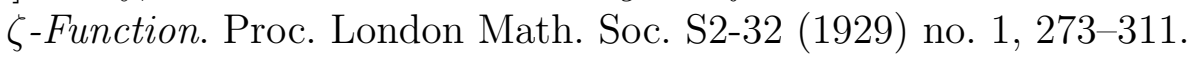

[Por] Porter, J.W. On a theorem of Heilbronn. Mathematika 22 (1975), no. 1, 20-28.

[Sou00] Soundararajan, K. Nonvanishing of quadratic Dirichlet L-functions at $s=\frac{1}{2}$. Ann. of Math. (2) 152 (2000), no. 2, 447-488.

[Sou07] Soundararajan, K. The fourth moment of Dirichlet L-functions. Analytic number theory, 239-246, Clay Math. Proc., 7, Amer. Math. Soc., Providence, RI, 2007.

[Tit] Titchmarsh, E.C. The theory of the Riemann zeta-function. Second edition. Edited and with a preface by D. R. Heath-Brown. The Clarendon Press, Oxford University Press, New York, 1986.

[Ton] Tonkov, T. The mean length of finite continued fractions. Papers presented at the Fifth Balkan Mathematical Congress (Belgrade, 1974). Math. Balkanica 4 (1974), 617-629. 
[Wat] Watt, N. Fourier coefficients of modular forms and eigenvalues of a Hecke operator. Funct. Approx. Comment. Math. 34 (2005), 27-146.

[WW] Whittaker, E.T.; Watson, G.N. A course of modern analysis. Reprint of the fourth (1927) edition. Cambridge Mathematical Library. Cambridge University Press, Cambridge, 1990 .

[YK] Yao, A.C.; Knuth, D.E. Analysis of the subtractive algorithm for greatest common divisors. Proc. Nat. Acad. Sci. U.S.A. 72 (1975), no. 12, 4720-4722.

[You11a] Young, M.P. The reciprocity law for the twisted second moment of Dirichlet Lfunctions. Forum Math. 23 (2011), no. 6, 1323-1337.

[You11b] Young, M.P. The fourth moment of Dirichlet L-functions. Ann. of Math. (2) 173 (2011), no. 1, 1-50.

[Zag] Zagier, D. Quantum modular forms, Clay Math Proceedings AMS 11(11), 659-675, 2010.

S. Bettin, Dipartimento di Matematica, Università di Genova; via Dodecaneso 35; 16146 Genova; Italy.

E-mail address: bettin@dima.unige.it

Dipartimento di Matematica, Università di Genova; via Dodecaneso 35; 16146 Genova; Italy. 